C. 3

\title{
LIBRARY
}

USE ONLY

\section{Procedure for Categorizing Ground Vehicles}

E. Alex Baylot and Burhman Q. Gates

November 2002

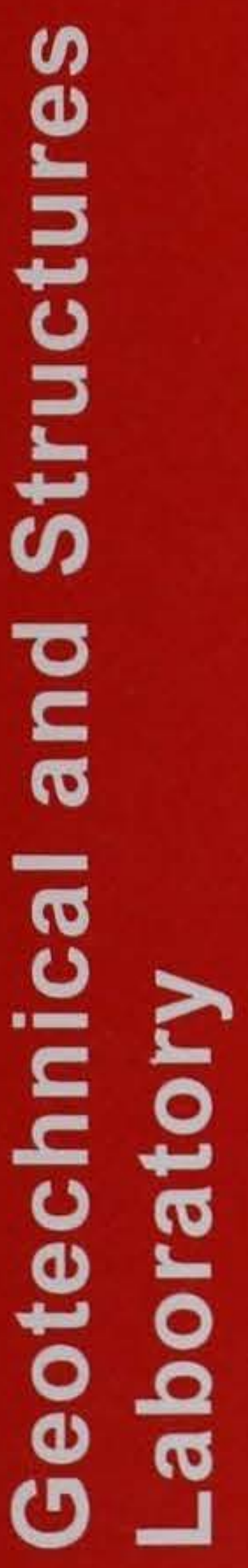




\section{US $=$ CE $=$ C Property of the}

Uniled States Government
ERDC/GSL TR-02-21

November 2002

TAT

E8

Procedure for Categorizing Ground Vehicles

by E. Alex Baylot, Burhman Q. Gates

TR-OZ-2I

Geotechnical and Structures Laboratory

U.S. Army Engineer Research and Development Center

C, 3 3909 Halls Ferry Road

Vicksburg, MS 39180-6199

Final report

Approved for public release; distribution is unlimited

Prepared for Office of Assistant Secretary of the Army for Acquisition, Logistics, and Technology Washington, DC 20310

and Army Models and Simulations Office Washington, DC 20310-0400

Research Library

USACE ERDC

Vickssburg, MS 


\section{Contents}

Preface

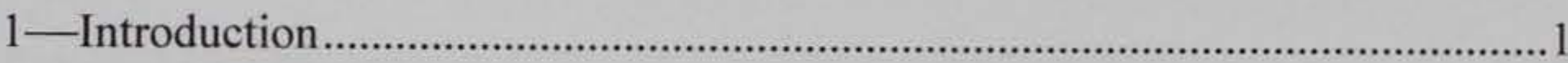

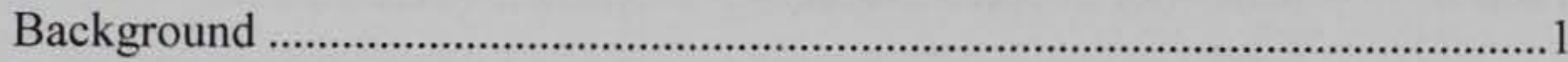

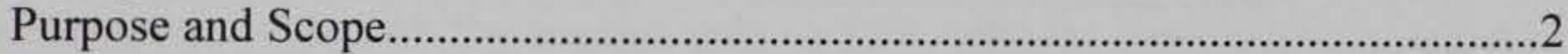

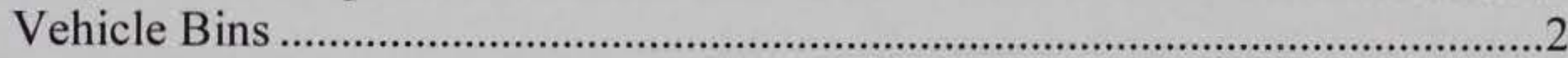

2-Approach to Vehicle Binning ........................................................................

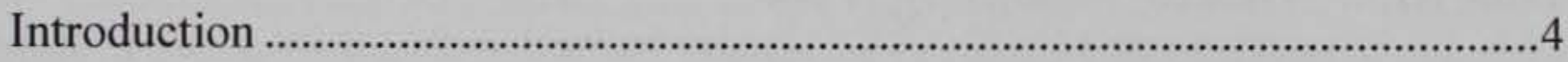

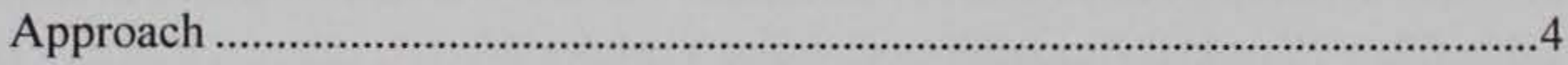

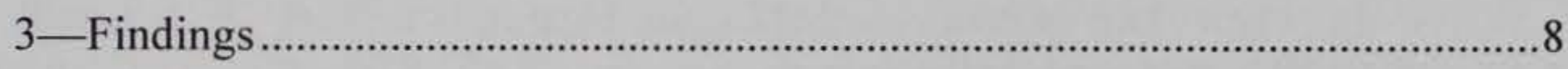

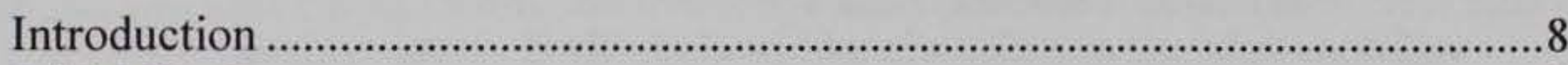

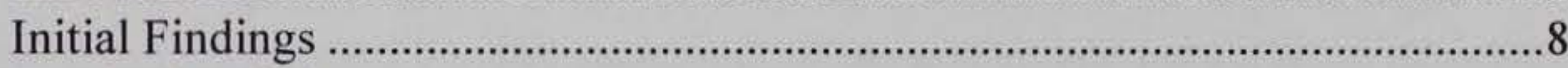

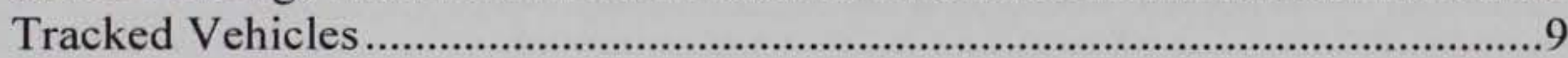

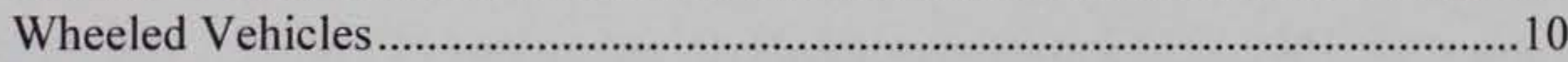

Wheeled Vehicles Towing Trailers ..................................................................12

Sensitivity of Bin Membership......................................................................13

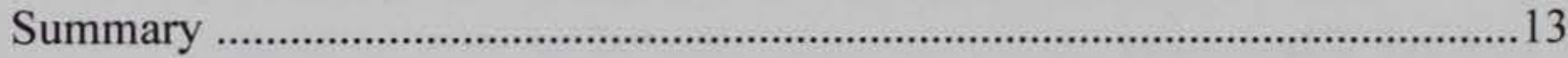

4-Bin Membership Procedure _.....................................................................15

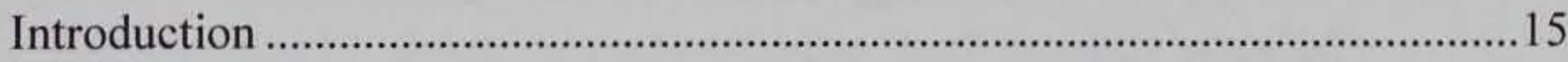

Procedure

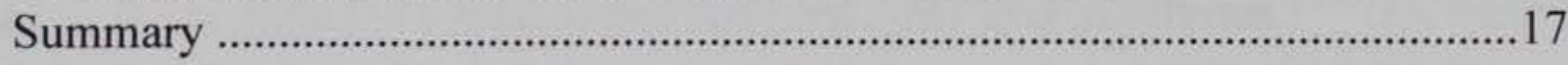

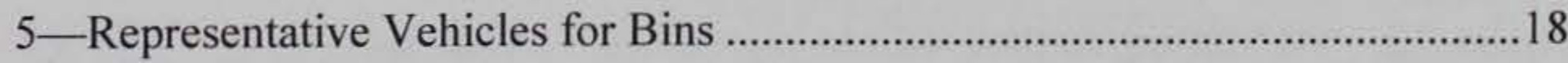

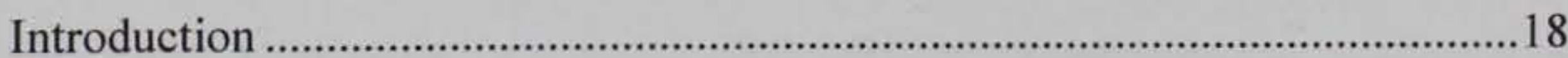

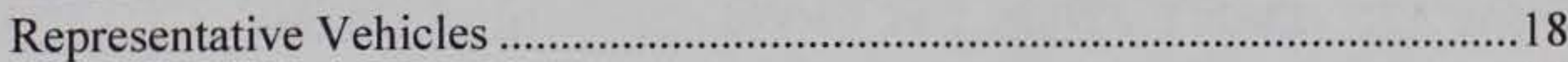

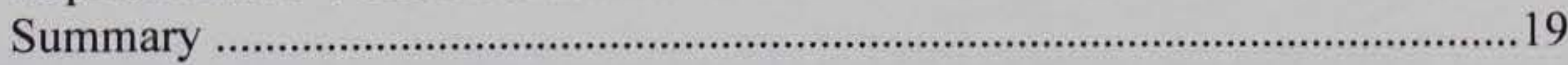

6-Conclusions and Recommendations................................................................20

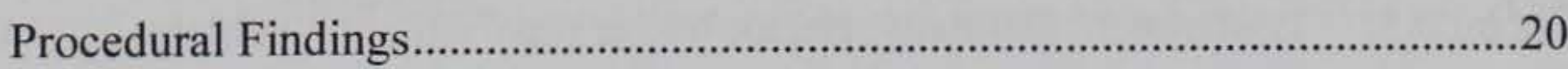

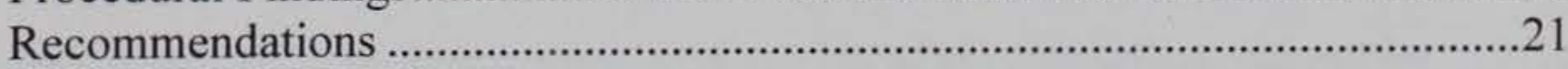


Appendix A: "Simulated Performance of WARSIM Bundle Representative Vehicles on Terrain in SWA, Europe, NEA, and Comparison of SRF and NRMM Derived Speed Predictions"

Appendix B: Tracked Vehicle Analysis ………................................................... B1

Appendix C: Wheeled Vehicle Analysis .............................................................

SF 298

\section{List of Tables}

Table 1. Sensitivity of Bin Membership to Attribute/Performance

Values

Table 2. Bins and Representative Vehicles ........................................... 19

Table B1. Tracked Vehicles, Bins 1-3 _................................................ B5

Table B2. Tracked Amphibious Combat Vehicles Bin $10 \ldots \ldots \ldots \ldots \ldots \ldots \ldots \ldots . . . \quad$ B6

Table C1. Wheeled Vehicles, Bins 4-6 .............................................. C6

Table C2. Wheeled Vehicles with Trailers, Bins 7-9 .............................. C7

Table C3. Wheeled Amphibious Combat Vehicle, Bin 11_...................... C7

\section{List of Figures}

Figure A1. Lauterbach, Germany, tracked representative vehicles............. A3

Figure A2. Al Mafraq, Jordan, tracked representative vehicles .................. A3

Figure A3. Dasht E Arzhan, Iran, tracked representative vehicles.............. A4

Figure A4. Cheorweon, South Korea, tracked representative vehicles ........ A4

Figure A5. Lauterbach, Germany, tractor/trailer representative vehicles .... A5

Figure A6. Al Mafraq, Jordan, tractor/trailer representative vehicles.......... A5

Figure A7. Dasht E Arzhan, Iran, tractor/trailer representative vehicles ..... A6

Figure A8. Cheorweon, South Korea, tractor/trailer representative vehicles 
Figure A9. Lauterbach, Germany, wheeled representative vehicles ........... A7

Figure A10. Al Mafraq, Jordan, wheeled representative vehicles................. A7

Figure A11. Dasht E Arzhan, Iran, wheeled representative vehicles ............ A7

Figure A12. Cheorweon, South Korea, wheeled representative vehicles....... A8

Figure A13. High mobility wheeled vehicle SRF, NRMM comparison ........ A9

Figure A14. Medium mobility wheeled vehicle SRF, NRMM comparison... A9

Figure A15. High mobility tractor/trailer SRF, NRMM comparison ............. A10

Figure A16. Medium mobility wheeled vehicle SRF, NRMM comparison... A10

Figure A17. High mobility tracked vehicle SRF, NRMM comparison.......... A11

Figure A18. Medium mobility tracked vehicle SRF, NRMM comparison .... A11

Figure A19. Low mobility tracked vehicle SRF, NRMM comparison .......... A12

Figure A20. BMP-2 simulated using Medium mobility SRFs and High

Mobility SRFs, and compared to NRMM computed BMP-2 performance. BMP-2 should be classified in the high mobility wheeled bundle

Figure A21. HEMTT simulated using Medium mobility SRFs and High Mobility SRFs, and compared to NRMM computed HEMTT performance. HEMTT should be classified in the medium mobility wheeled bundle ........................................................ A13

Figure A22. Performance of MTV, LMTV, and M1025A2. The MTV and LMTV should be reclassified as "high mobility wheeled" vehicles

Figure A23. High mobility wheeled SRF, NRMM comparison

Figure A24. High mobility wheeled SRF, NRMM comparison

Figure A25. High mobility wheeled SRF, NRMM comparison, computed using new "high mobility wheeled" (MTV) representative vehicle SRFs.

Figure A26. High mobility wheeled SRF, NRMM comparison, computed using new "high mobility wheeled" (MTV) representative vehicle SRFs.

Figure A27. High mobility wheeled SRF, NRMM comparison, computed using new "high mobility wheeled" (MTV) representative vehicle SRFs. 
Figure A28. High mobility wheeled vehicles simulated by NRMM with terrain in Lauterbach, Germany.

Figure A29. Low mobility tracked vehicle SRF, NRMM comparison

Figure A30. Low mobility tracked vehicle SRF, NRMM comparison using alternate vehicle SRFs

Figure A31. Low mobility tracked vehicle SRF, NRMM comparison using alternate vehicle SRFs

Figure A32. NRMM results for tracked representative vehicle in Lauterbach, Germany

Figure B1. Results of regression analysis for tracked vehicles (Iteration 1).

Figure B2. Results of paired t test for tracked vehicles (Iteration 1)

Figure B3. Results of regression analysis for tracked vehicles (Iteration 2)

Figure B4. Results of paired t test for tracked vehicles (Iteration 2).

Figure B5. Results of regression analysis for tracked vehicles (Iteration 3)

Figure B6. Results of paired t test for tracked vehicles (Iteration 3).

Figure C1. Results of regression analysis for wheeled vehicles (Iteration 1)

Figure C2. Results of paired t test for wheeled vehicles (Iteration 1)

Figure C3. Results of regression analysis for wheeled vehicles (Iteration 2)

Figure C4. Results of paired t test for wheeled vehicles (Iteration 2)

Figure C5. Results of regression analysis for wheeled vehicles (Iteration 3)

Figure C6. Results of paired t test for wheeled vehicles (Iteration 3)

Figure C7. Results of regression analysis for wheeled vehicles (Iteration 4)

Figure C8. Results of paired t test for wheeled vehicles (Iteration 4) 


\section{Preface}

Members of the staff of the U.S. Army Engineer Research and Development Center (ERDC), Geotechnical and Structures Laboratory (GSL), Engineer Systems and Materials Division (ESMD), Mobility Systems Branch (MSB), Vicksburg, MS, conducted the study analysis reported herein. The work was conducted under the combined sponsorships from the Office of Assistant Secretary of the Army for Acquisition, Logistics, and Technology, under the 62784/T40/154 project element, and the Army Models and Simulations Office (DAMO-ZS), under the project "Standard Mobility Suite for Variable Resolution Analysis and Cross-Model Consistency."

The study was conducted under the general supervision of Dr. David W. Pittman, Acting Director, GSL; Dr. Albert J. Bush III, Chief, ESMD; and Dr. David A. Horner, Chief, MSB. Mr. Erwin A. "Alex" Baylot, Jr., MSB, accomplished the overall report development; Messrs. Baylot and Burhman Q. Gates, Jr., MSB, prepared the report. Messrs. Peter Melick and Larry Martin, U.S. Army Materiel Systems Analysis Activity, served as independent reviewers. Additionally, Mr. Martin prepared Appendix A.

At the time of publication of this report, Dr. James R. Houston was Director of ERDC and COL John W. Morris III, EN, was Commander and Executive Director.

The contents of this report are not to be used for advertising, publication, or promotional purposes. Citation of trade names does not constitute an official endorsement or approval of the use of such commercial products. 


\section{Introduction}

\section{Background}

The Modeling and Simulation (M\&S) community models a large number of vehicle types. To ensure validity of these models, it's important to accurately represent vehicle mobility and logistical movements. Of course, the most accurate mobility representation would be to explicitly model each vehicle type. However, it is not practical to explicitly model every vehicle type in some applications-especially those simulating battalions and above. In those applications, vehicle classes are modeled instead of vehicles. When considering a particular vehicle, one must first determine which mobility category the vehicle falls into and then use the mobility of that category of vehicles to represent the trafficability of that vehicle. The following is a procedure for determining the appropriate mobility category or bin that represents a particular ground vehicle.

Twelve vehicle bins were devised to categorize vehicle movement. The first nine bins were originated by the U.S. Army Model and Simulation MOVE Standards Category Panel and the U.S. Army Materiel Systems Analysis Activity (AMSAA) for use in the WARSIM 2000 (Appendix A). ${ }^{1}$ The additional three bins were added as a result of the analysis detailed within this report. ${ }^{2}$ Bins were developed for three levels of tracked vehicle mobility (1-3), three levels of wheeled (i.e., truck) mobility (4-6), three levels of wheeled vehicles towing trailers (7-9), two types of Amphibious Combat Vehicles (ACV) (10-11), and a sub $500 \mathrm{~kg}$ All Terrain Vehicle (ATV), unmanned (12). ${ }^{3}$ During the course of this research, the Joint Warfare System (JWARS) adopted the first eleven bins with the twelfth to be added later. ${ }^{4}$ Bin membership was based on vehicle performance as modeled by the NATO Reference Mobility Model (NRMM) and

\footnotetext{
1 "Simulated Performance of WARSIM Bundle Representative Vehicles on Terrain in SWA, Europe, NEA, and Comparison of SRF and NRMM II Derived Speed Predictions," AMSAA, October 1998.

${ }^{2}$ WARSIM 2000 is a computer-based simulation with associated hardware and is the Army's next generation command and control training environment. WARSIM 2000 will replace, in priority order, a number of existing legacy training simulations.

${ }^{3}$ The use of the term amphibious is meant to include armored vehicles that can maintain buoyancy in water or have design characteristics similar to vehicles that swim. ${ }^{4}$ A Standards-Based Movement and Infrastructure Aggregation Methodology for Mobility Representation in Modeling and Simulation, USAERDC, September 2001.
} 
logical groupings. ${ }^{1}$ Also, included are identified vehicles serving as the representative member of each bin. The identified representative vehicles have been adopted by JWARS and closely match what was identified in the AMSAA study.

The procedure described in this document is to be used as a tool to consistently generically categorize vehicles and place them into bins. It is understood that for particular scenarios, some vehicles might be better represented in another bin. However, it is highly unlikely that it would be beyond the adjacent bin (i.e., low mobility versus high mobility).

Cross-validation with NRMM speed predictions was performed and there were no instances where the predicted speeds differed by more than one adjacent bin between NRMM and the procedure described herein. For 20 percent of the instances, there was disagreement by one adjacent bin and in half of those instances, the categorization was near the edges of adjoining bins.

\section{Purpose and Scope}

The purpose of this study was to standardize a set of ground vehicle bins, name the respective representative vehicles, and provide a repeatable procedure to categorized ground vehicles into representative bins suitable for simulation/analysis when the specific modeled representation of a given vehicle is not practical. The scope of this research was constrained to the findings of previous analyses offered by AMSAA and USAERDC, open source data found in Jane's Yearbooks, and prediction results using the NRMM with terrain cells found in Kuwait, Iran, North Korea, and Germany. This report documents the conduct and findings of this research.

\section{Vehicle Bins}

Practical design considerations of current and future brigade level and above wargame simulations limit the bin count to about nine. Additionally, experience has shown that three representations each of wheeled and tracked vehicles are sufficient in most simulations, especially for on-road movement. The exceptions include, but are not limited to, specially designed vehicles such as ACVs. The amphibious requirement on these vehicles drastically limits the design envelope in order to assure floatation and ingress/egress capability. Scenarios that would

\footnotetext{
${ }^{1}$ NRMM II is a computer-based set of algorithms designed to predict the steady state maximum speed for a vehicle operating in a given area. NRMM II is capable of producing on-road, off-road and gap-crossing predictions. In making a mobility analysis, NRMM II considers vehicle characteristics, driver capabilities, road and terrain factors, weather conditions, and natural obstacles. More specifically, NRMM II requires as input over 90 parameters. These include vehicle weights, width, height, ground clearance, wheeled/tracked, horsepower, soil description, surface condition, soil strength, vegetation, and parameters describing mobility influencing factors including; driver visibility (braking distance), driver endurance, current and recent weather conditions, and the placement and descriptions of naturally occurring obstacles.
} 
call for movement across a significant number of streams, rivers, and beaches would need to explicitly model the amphibious capability of ACVs.

Furthermore, future combat vehicles will evolve and push the design envelope of ACVs outward. Therefore, for future design and operational considerations, separate bins were set aside for wheeled and tracked ACVs to better capture their performance envelope. Other future combat vehicles will exploit the benefits of smaller, unmanned ATVs, and as such, a twelfth bin was created.

In the future, expansion of this documented bin system may be needed to cover a broader spectrum of systems. Such systems might include ground effect vehicles (air cushioned) or exotic systems such as crawlers, hoppers, walkers, etc. Analysts will need the capability to study the operational impacts of using such vehicles.

The twelve bins are:

A. High Mobility Tracked Vehicle.

B. Medium Mobility Tracked Vehicle.

C. Low Mobility Tracked Vehicle.

D. High Mobility Wheeled Vehicle.

E. Medium Mobility Wheeled Vehicle.

F. Low Mobility Wheeled Vehicle.

G. High Mobility Wheeled Vehicle with Towed Trailer.

H. Medium Mobility Wheeled Vehicle with Towed Trailer.

I. Low Mobility Wheeled Vehicle with Towed Trailer.

J. Tracked ACV.

$K$. Wheeled ACV.

L. Light ATV.

The approach taken in developing the bins as well as the findings, are given in the following sections. Details of the analysis are found in the Appendices. 


\section{Approach to Vehicle Binning}

\section{Introduction}

This chapter provides a description of the initial approach taken to develop a procedure to categorize vehicles into representative bins. Variations from this initial approach are documented in the following chapter.

The objective of the approach was to develop a mathematical formula for grouping vehicle types into the WARSIM 2000 vehicle bins plus additional bins if needed. Multivariate Linear Regression was chosen as the mathematical paradigm because of its simplicity and robustness.

\section{Approach}

Search current international literature and gather characteristic data for 100 or more vehicles of varying sizes, functions, and manufacturers that are representative of the world's most common military vehicles. ${ }^{1}$ Data collected were:

A. Combat vehicle weight or combined vehicle weight (including trailers) (kg).

B. Empty weight $(\mathrm{kg})$.

C. Power-to-weight ratio (computed when not given) (hp/t) $\{$ equal to CVW/engine power* 1000$\}$.

D. Ground clearance (minimum of vehicle or trailer) (m).

E. Maximum road speed (kph).

F. Fording depth (m).

\footnotetext{
${ }^{1}$ Jane's Yearbooks were used as the primary open source data.
} 
G. Maximum vertical obstacle (tracks only) (m).

H. Ground contact pressure (tracks only) $\left(\mathrm{kg} / \mathrm{cm}^{2}\right)$.

I. Amphibious (or design is characteristic of amphibious) (Yes or No).

J. Maximum gradient (\%).

$K$. Engine power (hp).

L. Manufacturing type code (1: USA, UK, Germany; 2: Former Warsaw Pact, Iraq, China, North Korea).

M. Primary use code (1: Truck; 2: ACV; 3 : Heavy Equipment Transporter).

The steps used to develop the vehicle bins included:

1. From the list of 100 or more wheeled and tracked vehicles considered for this analysis, randomly choose and separate the vehicles into three sets parsed as wheeled, wheeled with trailer, and tracked vehicles. Each set would have a maximum of 30 vehicles (target sample size for Gaussian statistical analysis).

2. For the selected vehicles having NRMM II vehicle data files, perform cross-country and on-road speed predictions using high resolution NRMM II terrain data under average normal conditions (winter conditions excluded) for both vehicle sets, in (a) Kuwait, (b) Iran, (c) North Korea, and (d) Germany. ${ }^{1}$

3. Compute measures of performance (MOP) using NRMM II (Version 2.6.3). For each vehicle on each of the terrains and roads considered, collect the average cross-country omni-directional speed (Xcc), average primary road omni-directional speed (Xpr), average secondary road omni-directional speed (Xsr), and average trail omnidirectional speed $(\mathrm{Xt})$. Preliminary results showed that most vehicles could not negotiate roughly 8 percent of the cross-country terrain under average normal soil conditions. Considering that a driver will not attempt to negotiate such terrain, only consider the best 90 percentile of

\footnotetext{
${ }^{1}$ The data sets have been commonly used over the past several decades for vehicle mobility analysis studies at the U.S. Army Engineer Research and Development Center. More specifically they are designated as geographic quadrants 5449IV (Kuwait), 6449III (Iran), $3222 \mathrm{III}$ (North Korea), and 5520 (Germany).
} 
the terrain. The MOPs are equivalent to the TRADOC mobility levels. They are defined and computed as follows:

$$
\begin{aligned}
& \text { Tactical High : YTH }=0.6 \times X c c+0.1 \times X p r+0.3 \times((X s r+X t) / 2) \\
& \text { Tactical Standard : YTs }=0.3 \times X c c+0.2 \times X p r+0.5 \times((X s r+X t) / 2) \\
& \text { Tactical Support : Yss }=0.15 \times X c c+0.3 \times X p r+0.55 \times((X s r+X t) / 2)
\end{aligned}
$$

4. Tactical high would be indicative of tracked vehicle movement, tactical standard would be indicative of wheeled vehicle movement, and tactical support would be indicative of wheeled vehicles with trailers.

5. Formulate a multi-variant linear regression equation for each of the three data sets from the independent variables. The independent variables include the readily obtainable vehicle characteristics given in step 1 and the dependent variables defined by the MOPs. Use a stepwise regression method to eliminate insignificant independent variables while maintaining an adjusted $\mathrm{r}^{2}$ value greater or equal to 0.85 . $^{1}$

6. Randomly choose five additional NRMM II wheeled vehicle files, five additional NRMM II wheeled (with trailer) vehicle files, and five additional NRMM II tracked vehicle files as cross-validation subsets of the NRMMII vehicle files. Using the data collected in step 4, compute the MOPs of the corresponding sets of five vehicles. Choosing five vehicles will provide about a $1 / 3$ portion for cross validation and $2 / 3$ portion for regression.

7. Use a two-tailed paired $t$ test with $\alpha=0.05$ level of significance ( $p=5$ percent probability) to statistically test whether the regression equations are suitable for predicting performance and thus capable of appropriately binning a vehicle. The metric $p$ is the probability of obtaining a larger $t$ statistic by chance alone if there is no difference between the paired sample means. The MSE of the NRMM II and regression-paired differences of the validation set must not exceed $5 \mathrm{kph}^{2}$.

8. After suitable results are obtained, organize and develop the bin membership criteria by using the samples to perform a cluster analysis based on the standard deviations of the MOPs. The bin membership will be determined by the predicted speed and the bin breakpoint. The breakpoint speed will be the halfway point between the predicted speeds

\footnotetext{
${ }^{1}$ As the mean square error of a regression procedure approaches zero, the adjusted ${ }^{2}$ approaches a value of one. Stepwise regression is an approach for selecting a subset of factors for a regression model. In an iterative fashion, the procedure removes input factors from the regression equation that do not show a greater than 0.10 probability of receiving a greater $F$ statistic. Thus, by design, the procedure will increase the adjusted $r^{2}$ towards one and reduce the mean squared error until no other factors meet the screening criteria. Root Mean Square (RMS) is the square root of Mean Square Error.
} 
of the two vehicles that adjoin one another, but are members of different bins as determined via the cluster analysis.

9. Compute the MOPs for the remaining vehicles that have no NRMM predictions or in those cases where a NRMM vehicle file does not yet exist and test sensitivity and organize all vehicles into their respective bins using the computed MOPs and binning criteria. 


\section{Findings}

\section{Introduction}

This chapter provides the initial findings using the approach and the results of building algorithms for categorizing tracked, wheeled, and wheeled vehicles towing trailers. Additionally, a sensitivity analysis was performed on the given algorithms. A summary of the findings is provided at the end of this chapter.

\section{Initial Findings}

A stepwise linear regression procedure was used to develop a multi-variant linear regression with independent variables characterizing the vehicle. After several iterations of the approach and with less-than-acceptable results, it was discovered that by removing the ACVs from the wheeled vehicle set, the Adjusted $r^{2}$ improved and a more logical organization of vehicles was evident. The ACVs have design tradeoffs that greatly distinguish them from other vehicles (i.e., swimming, small loads, ground clearance, and armored). Therefore, two unique bins for wheeled and tracked ACVs are recommended and all wheeled and tracked ACVs vehicles would belong to their respective bins.

Moreover, upon review by AMSAA, it was found that permitting the use of independent variables that produce nonsensical interactions in nature within the regression equation can cause counterintuitive results when all other independent variables remained unchanged. This was caused by the wrong sign being given to an independent variable during the formulation of the regression equation. While this might not necessarily have had a negative impact if the vehicle data were accurate enough for all attributes considered, it would negatively impact bin membership if one or two of the variables in question were assigned significantly inaccurate data. Therefore, to ensure the sensitivity of the regression equation reacted in a physically reasonable manner, the approach was modified so as not to consider any regression formulation that assigned counterintuitive numeric signs to independent variables. 


\section{Tracked Vehicles}

Unlike trucks and heavy equipment transporters, tracked vehicles travel offroad more often than on-road, and therefore any binning algorithm for tracked vehicles should give primary emphasis to off-road travel. Thus, $\mathrm{Y}_{\mathrm{TH}}$ (tactical high) is the most appropriate MOP.

After randomly selecting and excluding five vehicles out of the 19 available NRMM II tracked vehicle files, an automated backwards stepwise regression procedure was explored as a means to develop a multi-variant linear regression using independent variables to characterize the vehicle and $\mathrm{Y}_{\mathrm{TH}}$ as the dependent variable. However, the automated procedure was abandoned since it didn't allow enough degrees of freedom.

A supervised procedure was then initiated with the backwards stepwise method. Stepping through this procedure and eliminating the variables empty weight, power-to-weight ratio, ground clearance, maximum fording depth, amphibious, maximum gradient, and primary use yielded a fit with an adjusted $r^{2}$ of 0.97 and a rms of $1.27 \mathrm{kph}$ (Figure B1). Upon evaluating the cross-validation vehicle sample with the derived regression equation and paired $t$ test, the computed $p$ was equal to 9.0 percent probability (Figure B2) and a MSE of $5.7 \mathrm{kph}^{2}$. Thus, since $p$ was greater than $\alpha(0.05$ or 5 percent $)$, it cannot be refuted that the samples are of the same population, but since the MSE was greater than $5 \mathrm{kph}^{2}$, the procedure was ineffective.

A forward stepwise linear regression procedure was then initiated. By using the described method, the maximum road speed was entered as the only independent variable yielding an adjusted $r^{2}$ value of 0.886 and a rms of $2.59 \mathrm{kph}$ (Figure B3). Upon evaluating the five vehicle cross-validation sample with the derived regression equation and paired $t$ test, the computed $p$ was equal to 34.2 percent probability (Figure B4) and a MSE of $6.6 \mathrm{kph}^{2}$. Thus, since $p$ was greater than $\alpha$, it cannot be refuted that the samples are of the same population, however, since the MSE was greater than $5 \mathrm{kph}^{2}$, the procedure was ruled ineffective.

A supervised procedure was then initiated with the forward stepwise method. Stepping through this procedure and adding the variable power-to-weight ratio showed a slight improvement with physically reasonable interactions. Adding just combined weight or power or combinations of each caused counterintuitive interactions that would cause the values of the dependent variable to behave in a manner that is reverse of what is physically probable. Other variables offered insignificant improvements to the regression fit. The resulting fit yielded an adjusted $\mathrm{r}^{2}$ of 0.887 and a rms of $2.58 \mathrm{kph}$ (Figure B5). Upon evaluating the cross-validation sample with the derived regression equation and paired $t$ test, the computed $p$ was equal to 50.2 percent probability (Figure B6) and a MSE of $4.99 \mathrm{kph}^{2}$. Thus, since $p$ was greater than $\alpha$, it cannot be refuted that the samples are of the same population, and since the MSE was less than $5 \mathrm{kph}^{2}$, the procedure was effective. 
The predicted speed from the regression model was used to select an appropriate bin based on a statistical cluster analysis balancing mean speed and standard deviations. The analysis yielded the following results:

\begin{tabular}{ccc} 
Cluster & $\frac{\text { Means }}{(\mathrm{kph})}$ & $\frac{\text { Cluster Standard }}{\text { Deviations }(\mathrm{kph})}$ \\
\hline 1 & 32.5 & 2.67 \\
2 & 23.7 & 1.40 \\
3 & 9.1 & 0.0
\end{tabular}

The results of the cluster analysis placed only one vehicle in the lowest mobility bin. Upon review of the cluster results by SMEs, it was judged to be unsatisfactory and it was decided that a more logical grouping of the clusters could be achieved by shifting the breakpoint speed from 15.4 to $26.3 \mathrm{kph}$ for the lower and middle cluster and leaving the breakpoint speed at 31.2 for the middle and upper cluster. No other changes were made to the cluster membership. These changes allowed more agreement with the regression predictions and NRMM II predictions insofar as bin membership. The adjustments yielded the following results:

$\begin{array}{cccc}\frac{\text { Cluster }}{1} & \frac{\text { Means }}{(\mathrm{kph})} & \frac{\text { Cluster Standard }}{\text { Deviations }(\mathrm{kph})} & \frac{\text { Breakpoint }}{(\mathrm{kph})} \\ 2 & \frac{13.7}{33.7} & 1.23 & >31.2 \\ 3 & 29.2 & 4.29 & >26.3<31.2 \\ 22.2 & & <26.3\end{array}$

Results of the binning procedure are provided in the last column of the Appendix B, Table B1. Table B2 provides the tracked ACVs used for this analysis.

\section{Wheeled Vehicles}

Wheeled vehicles travel on-road more often than off-road, and therefore, any binning algorithm for wheeled vehicles should give primary emphasis to on-road travel. Thus, $\mathrm{Y}_{\mathrm{TS}}$ (tactical standard) is the most appropriate MOP.

After randomly selecting and excluding 5 vehicles out of the 15 available NRMM II wheeled vehicle files, an automated backwards stepwise regression procedure was used to develop a multi-variant linear regression using independent variables to characterize the vehicle and $\mathrm{Y}_{\mathrm{TS}}$ as the dependent variable. As with the tracked vehicles, the procedure proved ineffective due to the limiting degrees of freedom.

A forward stepwise linear regression procedure was used to develop a multivariant linear regression using independent variables to characterize the vehicle and the $\mathrm{Y}_{\mathrm{TS}}$ as the dependent variable. Ensuring physically reasonable interactions occurred and using the described method, the power-to-weight ratio, maximum gradient, and mfg type code were entered as the only independent variables 
yielding an adjusted $\mathrm{r}^{2}$ value of 0.777 and a rms of $3.59 \mathrm{kph}$ (Figure $\mathrm{C} 1$ ). Upon evaluating the cross-validation vehicle sample with the derived regression equation and paired $t$ test, the computed $p$ is equal to a 76.2 percent probability level (Figure C2) and a MSE of $76.2 \mathrm{kph}^{2}$. Thus, since $p$ was greater than $\alpha$, it cannot be refuted that the samples were of the same population, but since the MSE was greater than $5 \mathrm{kph}^{2}$, the procedure was ruled ineffective.

Following the results of the forward stepwise linear regression procedure used for tracked vehicles, the maximum road speed was entered as the only independent variable. This resulted in an adjusted $r^{2}$ of -0.03 and a rms of $7.71 \mathrm{kph}$ (Figure C3). Upon evaluating the five cross-validation vehicle sample with the derived regression equation and paired $t$ test, the computed $p$ is equal to a 50.2 percent probability level (Figure C4) and a MSE of $28.7 \mathrm{kph}^{2}$. Thus, since $p$ was greater than $\alpha$ it cannot be refuted that the samples are of the same population, but since the MSE was greater than $5 \mathrm{kph}^{2}$, the procedure was ruled ineffective.

A supervised procedure was then initiated with the forward stepwise method. Stepping through this procedure and adding the variable power-to-weight ratio, caused physically unreasonable interactions with maximum road speed. In other words, increasing maximum road speed caused a decrease in $\mathrm{Y}_{\mathrm{TS}}$. Continuing with the procedure, adding maximum gradient and power improved the regression fit and maintained physically reasonable interactions. The resulting fit yielded an adjusted $\mathrm{r}^{2}$ of 0.813 and a rms of $3.29 \mathrm{kph}$ (Figure C5). Upon evaluating the 5 vehicle cross-validation sample with the derived regression equation and paired $t$ test, the computed $p$ was equal to 10.4 percent probability (Figure C6) and a MSE of $120.8 \mathrm{kph}^{2}$. Thus, since $p$ was greater than $\alpha$, it cannot be refuted that the samples are of the same population, but since the MSE was greater than $5 \mathrm{kph}^{2}$, the procedure was also ineffective.

Through these three iterations, the procedure was not successful with using the MOP $\mathrm{Y}_{\mathrm{TS}}$. Although it was previously postulated that $\mathrm{Y}_{\mathrm{TS}}$ should be the MOP for wheeled vehicles, it didn't work for the described method. Thus, Y was then used as the MOP.

Following the course taken previously, similar results followed. Stepping through the procedure and using the variables power-to-weight ratio and maximum gradient yielded favorable and physically reasonable results. The resulting fit yielded an adjusted $r^{2}$ of 0.687 and a rms of $4.62 \mathrm{kph}$ (Figure C7). Upon evaluating the five vehicle cross-validation sample with the derived regression equation and paired $t$ test, the computed $p$ was equal to 76.4 percent probability (Figure C8) and a MSE of $29.5 \mathrm{kph}^{2}$. Thus, since $p$ was greater than $\alpha$, it could not be refuted that the samples are of the same population, but since the MSE was greater than $5 \mathrm{kph}^{2}$, the procedure was also ineffective.

The significant difference in the results of the previous iteration (Figure C7 \& C 8) and iteration 2 (Figure C3 \& C4) was that the fit was about the same for the data set used for the regression and the data set used for cross-validation. Furthermore, since much greater speeds are obtainable with $\mathrm{Y}_{\mathrm{Ss}}$ as opposed to 
$\mathrm{Y}_{\mathrm{TH}}$, the criterion for obtaining a MSE less than $5 \mathrm{kph}^{2}$ was relaxed and it was decided that a MSE of $29.5 \mathrm{kph}^{2}$ is acceptable.

The predicted speed from the regression model was used to select an appropriate bin based on a statistical cluster analysis balancing mean speed and standard deviations. The analysis yielded the following results:

\begin{tabular}{ccc} 
Cluster & $\frac{\text { Means }}{(\mathrm{kph})}$ & $\frac{\text { Cluster Standard }}{\text { Deviations }(\mathrm{kph})}$ \\
\hline 4 & $\frac{51.2}{4.75}$ \\
5 & 42.3 & 2.29 \\
6 & 33.9 & 3.32
\end{tabular}

Upon review of the cluster results by subject-matter-experts (SMEs), it was decided that a more logical grouping of the clusters could be achieved by expanding the membership of the fastest cluster to include the top three members of the middle cluster. In turn, this shifted the breakpoint speed to $42.9 \mathrm{kph}$. The breakpoint between the two bottom clusters was unchanged. The adjustments yielded the following results:

\begin{tabular}{|c|c|c|c|}
\hline Clucter & $\frac{\text { Means }}{(k n h)}$ & Cluster Standard & $\underline{\text { Breakpoint }}$ \\
\hline 4 & $\frac{\text { (крі11) }}{47.3}$ & $\frac{\text { Deviations (kpin) }}{4.2}$ & $>42.9$ \\
\hline 5 & 40.8 & 0.98 & $>38.2<42.9$ \\
\hline 6 & 33.9 & 3.32 & $<38.2$ \\
\hline
\end{tabular}

The data used for predicting the $Y_{\mathrm{TS}}$ and $Y_{\mathrm{SS}}$ speeds of 29 vehicles are found in Appendix C Table $\mathrm{C}$ 1. The predicted $\mathrm{Y}_{\mathrm{TS}}$ and $\mathrm{Y}_{\mathrm{SS}}$ speeds are given as well and are sorted from highest speed to lowest speed. Because there were not a sufficient number of NRMM wheeled vehicle files where the vehicle was not towing a trailer, 29 rather than 30 vehicles were selected. Table $\mathrm{C} 3$ provides the wheeled ACVs used for this analysis.

\section{Wheeled Vehicles Towing Trailers}

As discussed above, trucks travel on-road more often than off-road. This is especially true for trucks towing trailers. Therefore any binning algorithm for such vehicles should give even more emphasis to on-road travel than provided by tactical standard. The MOP most appropriate would be tactical support, $\mathrm{Y}_{\mathrm{SS}}$.

However, because there were an insufficient number of NRMM vehicle files (7) to build a regression equation and perform cross validation, an expert procedure was employed. The most obvious criteria for selecting members to the lowest mobility wheeled with trailer was determining whether the vehicle was a heavy equipment transporter, or primary use code 3 . Thus, vehicles having a primary use code of 3 belong to Bin 9 . Their combined vehicle weights were all greater than $60,000 \mathrm{~kg}$. 
The next step was to build breakpoint criteria for the remaining vehicles. From subject-matter-expert experience, combined vehicle weight and power are historically good criteria for categorizing vehicles. Thus, the power-to-weight ratio is a logical breakpoint criterion. The power-to-weight ratios given in Table B3 show a range of 6.1 to 21.6. From past AMSAA and ERDC analysis, the MTV with trailer was found to be a high mobility vehicle and M985 with trailer was found to be a medium mobility vehicle (see Appendix A). Thus, the breakpoint should be between their respective values. This is set at a 10.0 powerto-weight ratio. Results of the binning procedure are provided in the last column of Appendix C, Table C2.

\section{Sensitivity of Bin Membership}

Although it is certainly desired that data values of vehicle attributes and stated performance from various sources be equal, the reality is they are not. Variation occurs for different reasons and can be attributed to whether the source is classified, unclassified, undocumented modifications, data collection errors, etc. Thus, it was important to examine the sensitivity of the binning algorithms to variations. Sensitivity was given as the percent of vehicles that changed bins as a result of a percent change in attribute/performance values. The results are given in Table 1.

\begin{tabular}{|c|c|c|c|c|}
\hline \multicolumn{5}{|c|}{$\begin{array}{l}\text { Table } 1 \\
\text { Sensitivity of Bin Membership to Attribute/Performance Values }\end{array}$} \\
\hline $\begin{array}{l}\text { Attribute/Performance } \\
\text { Descriptions }\end{array}$ & $\%$ Variation & $\begin{array}{l}\% \text { Change } \\
\text { Tracked } \\
\text { Vehicles } \\
\end{array}$ & $\begin{array}{l}\% \text { Change } \\
\text { Wheeled } \\
\text { Vehicles }\end{array}$ & $\begin{array}{l}\% \text { Change } \\
\text { Wheeled } \\
\text { Vehicles } \\
\text { w } / \text { Trailers }\end{array}$ \\
\hline \multirow{3}{*}{ Combat Weight (Gross Combined) } & 5 & 3 & 10 & ב11 \\
\hline & 10 & 3 & 21 & 11 \\
\hline & 20 & 3 & 35 & 22 \\
\hline \multirow{3}{*}{ Power } & 5 & 0 & 10 & 11 \\
\hline & 10 & 3 & 21 & 22 \\
\hline & 20 & 23 & 35 & 33 \\
\hline \multirow{3}{*}{ Power-To-Weight Ratio } & 5 & 0 & 7 & 11 \\
\hline & 10 & 3 & 28 & 22 \\
\hline & 20 & 3 & 41 & 33 \\
\hline \multirow{3}{*}{ Maximum Road Speed } & 5 & 3 & N/A & N/A \\
\hline & 10 & 37 & N/A & N/A \\
\hline & 20 & 60 & N/A & N/A \\
\hline \multirow{3}{*}{ Maximum Gradient } & 5 & N/A & 0 & N/A \\
\hline & 10 & N/A & 0 & N/A \\
\hline & 20 & N/A & 10 & N/A \\
\hline
\end{tabular}

\section{Summary}

Both automated and supervised backward stepwise linear regression methods failed to lead to a set of vehicle parameters that could be used to categorize tracked vehicles of comparable performance into bins. A supervised forward 
stepwise linear regression procedure was the best method for determining the best set of vehicle characteristic variables to use for categorizing tracked vehicles.

For wheeled vehicles, it was not possible to determine bin membership with any of the regression methods using tactical standard as a MOP. The tactical support MOP was used instead of tactical standard as a means of assessing bin selection. Using $\mathrm{Y}_{\mathrm{SS}}$ and a relaxed MSE, a supervised forward stepwise linear regression was used to identify power-to-weight ratio and maximum gradient as vehicle parameters that best discriminated wheeled vehicles into bins.

The two-tailed $t$ test was ineffective in distinguishing between good and bad regression fits due to the limiting degrees of freedom and 95 percent criterion. The 95 percent criterion is a common but a high standard for refuting statistical claims. Thus, by default MSE was the only criterion used for goodness of fit.

For wheeled vehicles towing trailers, SME opinion was consulted since there were not enough NRMM vehicle files with towed trailers to form an adequate control group for comparison. Primary use is first used to screen low mobility wheeled towing vehicles from the others. Then, power-to-weight ratio was used to distinguish between medium and high mobility wheeled vehicles towing a trailer.

In regard to the sensitivity analysis, the tracked algorithm is the least sensitive of the three and is more in agreement with NRMM insofar as MOP. The results of the sensitivity analysis showed that the results of the tracked algorithm was less sensitive when varying weight and power-to-weight ratio, about the same for power, and most sensitive when varying maximum road speed.

For wheeled vehicles, agreement is not as good as with tracked and there is a wider variance with the predictions of the algorithm and NRMM. The wheeled vehicles were the most sensitive to changes in attribute/performance values. The wheeled vehicles with trailers in tow are fairly sensitive but not as much as just wheeled vehicles.

It could not be verified whether the sensitivity of the results as given was correct without conducting substantial research beyond the scope of this analysis, but the results do yield guidance as to how accurate the input data should be. As stated in the introduction, analysis requiring highly accurate mobility predictions should not use these surrogate vehicles to represent particular vehicles. 


\section{Bin Membership Procedure}

\section{Introduction}

This chapter provides the algorithms for categorizing vehicles into the bins that best approximate a vehicle's mobility. A summary of the findings is provided at the end of this chapter.

\section{Procedure}

If the vehicle is tracked and its combat vehicle weight $>500 \mathrm{~kg}$, then go to step A. If the vehicle is wheeled and its combat vehicle weight $>500 \mathrm{~kg}$, then go to step B. Else, vehicle is a light ATV and thus go to step C.

A. Tracked Vehicles (Bins 1-3,10):

(1) Collect, at a minimum, the following information on a tracked vehicle. ${ }^{1}$ If the vehicle is an ACV then go to step 2.

Combat vehicle weight $(\mathrm{kg})$

Power (hp)

Maximum road speed $(\mathrm{kph})$

or

Power-to-weight ratio (hp/ton)

Maximum road speed ( $\mathrm{kph})$

Note: Published power-to-weight ratios do not always equal the ratio of the published power and combat vehicle weight (all multiplied by 1,000$)$, but are close in value.

(2) If the primary use code is equal to 2, then place the vehicle in Bin 10.

\footnotetext{
${ }^{1}$ Jane's Yearbooks were used as the primary open source data.
} 
(3) Otherwise, use the following equation to tactical high speed, $Y_{\mathrm{TH}}$ (kph).

$\mathrm{Y}_{\mathrm{TH}}=2.4+0.229 *$ (power-to-weight ratio $)+0.382 *$ maximum road speed

or

$\mathrm{Y}_{\mathrm{TH}}=2.4+0.229 *($ power $) /($ combat vehicle weight $* 0.00111)+$ $0.382 *$ maximum road speed

Note: Published power-to-weight ratios do not always equal the ratio of the published power and combat vehicle weight (all multiplied by $1 \mathrm{~kg} / 0.00111 \mathrm{ton})$, but are close in value.

(4) Use the value of YTH to select the vehicle bin using:

Bin 1 $\mathrm{Y}_{\mathrm{TH}} \geq 31.2$

Bin 2 $\mathrm{Y}_{\mathrm{TH}} \geq 26.3$ and $\mathrm{Y}_{\mathrm{TH}}<31.2$

Bin 3 $\mathrm{Y}_{\mathrm{TH}}<26.3$

B. Wheeled Vehicles (Bins 4-9,11):

(1) Collect the following information on a wheeled vehicle. ${ }^{1}$ If the vehicle is an ACV then go to step 2.

Maximum gradient (\%)

Primary use code (1: Truck; 2: ACV; 3: Heavy Equipment Transporter)

Trailer attached (True/False)

Combat vehicle weight $(\mathrm{kg})$

Power (hp)

or

Maximum gradient (\%)

Primary use code (1: Truck; 2: ACV; 3: Heavy Equipment Transporter)

Trailer attached (True/False)

Power-to-weight ratio (hp/ton)

(2) If the primary use code is equal to 2 , then place the vehicle in Bin 11.

(3) If a trailer is not attached to the wheeled vehicle, then use the following equation to bin:

\footnotetext{
${ }^{1}$ Jane's Yearbooks were used as the primary open source data.
} 
$\mathrm{Y}_{\mathrm{SS}}=1.20+1.258 *$ (power-to-weight ratio) $+0.338 *$ maximum gradient

or

$\mathrm{Y}_{\mathrm{SS}}=1.20+1.258 *($ power $) /($ combat vehicle weight $* 0.00111)+$ $0.338 *$ maximum gradient

(4) Use the value of $Y_{S S}$ to select the vehicle bin using:

Bin 4 $\mathrm{Y}_{\mathrm{SS}} \geq 42.9 \mathrm{kph}$

Bin 5 $\mathrm{Y}_{\mathrm{SS}} \geq 38.2 \mathrm{kph}$ and $\mathrm{Y}_{\mathrm{SS}}<42.9 \mathrm{kph}$

Bin 6 $\mathrm{Y}_{\mathrm{SS}}<38.2 \mathrm{kph}$

(5) If a trailer is attached and the primary use code is equal to 3 or the combined vehicle weight exceeds $60,000 \mathrm{~kg}$, then place the vehicle in Bin 9.

(6) Otherwise bin as follows:

Bin 7 Power-to-Weight Ratio $\geq 10.0$

Bin 8 Power-to-Weight Ratio $<10.0$

\section{Light ATV (Bin 12):}

If vehicle is light ATV or less than $500 \mathrm{~kg}$, then place in Bin 12 .

\section{Summary}

This chapter provided a step-by-step procedure for deciding the appropriate mobility category and bin in which to place a vehicle. The algorithms were written in such a manner as to facilitate computer programming. 


\section{Representative Vehicles for Bins}

\section{Introduction}

This chapter enumerates the vehicles chosen to represent each of the bins and gives the rationale for choosing them as representatives. Every effort was made to use vehicles already serving as representative vehicles in the modeling and simulation community, wherever possible.

\section{Representative Vehicles}

Following vehicle performance, the next principle requirement for selecting a representative vehicle was the availability of accurate data and SME/user familiarity with the vehicle. Thus, selecting U.S. made vehicles was imperative regardless of whether the vehicle's performance falls near the middle of a given bin, although that was preferred when available.

The vehicles representing Bins 1, 3, 4, 7, 9 are those listed or recommended in the AMSAA study for cross-country vehicle binning. The focus of the AMSAA study was to verify choices of vehicles to represent mobility bundles of WARSIM vehicles operating in cross-country environments. Changes in representation to the original nine were brought about by the need for additional bins and results of a JWARS study. In this latter study, emphasis was given to logistical movement rather than cross-country movement, thus on-road consideration became important.

In keeping with the described changes, the M270 replaced the M113A3 in Bin 2 when it moved to the representative vehicle for Bin 10; the M985 replaced the M935 for Bin 5, likewise for Bin 8; and the M917 replaced the M911 for Bin 6. The LAV25 serving as the Interim Brigade Vehicle was the logical choice for Bin 11, and finally for Bin 12 the ATV representative was chosen for its current widespread use in the U.S. and its size being similar to anticipated future vehicle systems.

Although, Kawasaki is a foreign-owned company, it has been producing ATVs in the U.S. since the mid 1970's. These choices for bin representatives are 
further corroborated by the adoption of the same vehicles as mobility category representatives in JWARS.

Per the results of the aforementioned stated references and results described in this document, the twelve bins and their corresponding representative vehicles are given in Table 2. References are given as to whether they are representative in WARSIM (or later refined by AMSAA for WARSIM), CCTT-SAF, and JWARS.

\begin{tabular}{|c|c|c|c|c|c|}
\hline No. & Bin Name & Vehicle & $\begin{array}{l}\text { WARSIM/ } \\
\text { AMSAA } \\
\text { Recomd. }\end{array}$ & CCTT & JWARS \\
\hline 1 & High Mobility Tracked Vehicle & M1A1 & $\mathrm{X}^{\prime}$ & $\mathrm{x}$ & $\mathrm{x}$ \\
\hline 2 & $\begin{array}{l}\text { Medium Mobility Tracked } \\
\text { Vehicle }\end{array}$ & M270 MLRS & diff $^{2}$ & $N / A^{3}$ & $x$ \\
\hline 3 & Low Mobility Tracked Vehicle & M60 AVLB & $\mathrm{x}$ & $\mathrm{x}$ & $\bar{x}$ \\
\hline 4 & High Mobility Wheeled Vehicle & M1084 MTV & $\bar{x}$ & diff & $\bar{x}$ \\
\hline 5 & $\begin{array}{l}\text { Medium Mobility Wheeled } \\
\text { Vehicle }\end{array}$ & M985 HEMTT & diff & $\mathrm{x}$ & $\mathrm{x}$ \\
\hline 6 & Low Mobility Wheeled Vehicle & M917 Dumo Truck & diff & diff & $x$ \\
\hline 7 & $\begin{array}{l}\text { High Mobility Wheeled Vehicle } \\
\text { w/Towed Trailer }\end{array}$ & M1084/M1094 & $\mathrm{x}$ & N/A & $\mathrm{x}$ \\
\hline 8 & $\begin{array}{l}\text { Medium Mobility Wheeled } \\
\text { Vehicle w/Towed Trailer }\end{array}$ & M985/M989 & diff & N/A & $\mathrm{x}$ \\
\hline 9 & $\begin{array}{l}\text { Low Mobility Wheeled Vehicle } \\
\text { w/Towed Trailer }\end{array}$ & M911/M747 HET & $\mathrm{x}$ & N/A & $\mathrm{x}$ \\
\hline 10 & Tracked ACV & M113A2 & N/A & N/A & $\mathrm{x}$ \\
\hline 11 & Wheeled ACV & LAV25 & N/A & N/A & $\bar{x}$ \\
\hline 12 & Light ATV & $\begin{array}{l}\text { Kawasaki ATV } \\
\text { (high shock) }\end{array}$ & N/A & N/A & $\mathrm{x}$ \\
\hline
\end{tabular}

\section{Summary}

This chapter identified other studies and simulations used as references in choosing representative vehicles for each of the mobility categories as well as the findings of this research. Every effort was made to build on past research, select U.S. made vehicles, and bring the modeling and simulation community together on choices for representative vehicles. 


\section{Conclusions and Recommendations}

In summary, this report presents the procedures and findings of categorizing ground vehicles while providing the $M \& S$ community with a set of common categories and common representative vehicles. The procedure successfully used currently available parameterizations for ground vehicles and then used a subset of those vehicles to represent other vehicles with similar mobility performances. This was accomplished by defining the categorization, devising a practical binning system, and sorting the vehicles into those bins. A sensitivity analysis was performed to evaluate the robustness of the categorization mechanism against changes in the values of vehicle input parameters. Existing bins and representative vehicles were taken from WARSIM 2000 and JWARS. New bins were named for vehicles that defied classification into those bins. The resulting set of bins is provided in this report and includes the new groups of amphibious tracked, amphibious wheeled vehicles, and small vehicles $(<500 \mathrm{~kg})$.

\section{Procedural Findings}

A supervised forward stepwise linear regression procedure was the best method for determining the best set of vehicle characteristic variables to use for categorizing tracked vehicles when tied to the MOP tactical high. The same regression procedure was not suitable for wheeled vehicles when tied to the MOP tactical standard; however the MOP tactical support was suitable.

A regression procedure could not be used for wheeled vehicles towing trailers since there were not enough NRMM vehicle files with towed trailers to form an adequate control group for comparison. Thus, SME opinion was consulted. Primary use is first used to screen low mobility wheeled towing vehicles from the others. Then, power-to-weight ratio was used to distinguish between medium and high mobility wheeled towing vehicles. The remaining bins, amphibious tracked, amphibious wheeled only required differentiation as to whether the amphibious armored combat vehicle is tracked or wheeled, and finally for the ATV as to whether the vehicle's weight was less than $500 \mathrm{~kg}$.

A clustering technique was used to divide the tracked and wheeled groups into three bins each. The technique called for balancing the standard deviations 
of the pertinent MOPs from one cluster to another. For practical considerations, the criteria for bin membership were slightly adjusted to allow for the inclusion of existing or well-known representative vehicles.

Each of the categorization formulae was tested for sensitivity to changes in the values of input parameters. These results showed that the binning of tracked vehicles was less sensitive to changes in the values of vehicle parameters than for wheeled vehicles. Wheeled vehicles with towed trailers were also sensitive to vehicle parameter changes but not as sensitive as wheeled vehicles alone. These sensitivities for wheeled vehicles are not unexpected since the design of wheeled vehicles is less specialized than tracked vehicles and wheeled vehicles are expected to operate on a broader set of off-road and on-road conditions.

\section{Recommendations}

Based on the findings of this research, it is recommended that the 12 bins for categorizing ground vehicles be accepted as a standard for the M\&S community and be accepted for the U.S. Army Standards Repository System and the M\&S Resource Repository. It is further recommended that current and future wargame simulations and decision support systems adopt the categorization procedure and bins documented within this report.

Further refinement of this procedure may be accomplished by obtaining a larger set of vehicle data and predicting mobility on a larger set of terrain databases. Additionally, prior to analysis or during the course of simulation, allowing the flexibility of changing the representative vehicles of bins as a function of terrain conditions and features (i.e., plains, hills, obstacles) would better model the performance of a particular vehicle without being vehicle specific. This is recommended as the next level of resolution increase. 
Appendix A

\author{
"Simulated Performance of \\ WARSIM Bundle Representa- \\ tive Vehicles on Terrain in \\ SWA, Europe, NEA, and Com- \\ parison of SRF and NRMM \\ Derived Speed Predictions"
}




\title{
"Simulated Performance of WARSIM Bundle Representative Vehicles on
}

\author{
Terrain in SWA, Europe, NEA, and Comparison of SRF \\ and NRMM Derived Speed Predictions"
}

Introduction. This paper documents an analysis of the comparative performance of the vehicles chosen to represent the WARSIM mobility bundles, and the simulation of vehicle speed using the WARSIM Speed Reduction Factor (SRF) method. The purpose of the analysis was to verify that the vehicles chosen to represent the WARSIM mobility bundles are reasonable choices, and representative of these bundles, and to demonstrate the WARSIM SRF method of speed computation and it's accuracy. The scope of the analysis was limited due to time constraints.

First, the performance of the representative vehicles in each category (wheeled, tracked, and tractor-trailers) as predicted by the NATO Reference Mobility Model (NRMM) was evaluated by running the high, medium, and low mobility representative vehicles on terrain in four different regions of the world (Lauterbach Germany, Al Mafraq Jordan, Dasht E Arzhan Iran, and Cheorweon South Korea). Vehicle speed profiles for the high, low, and medium representative vehicles were then plotted and compared.

The second part of the analysis evaluates the WARSIM SRF method of speed computation. Because of time constraints the analysis was limited to one terrain, the Lauterbach region of Germany. Speeds for a vehicle chosen from the WARSIM mobility bundling document over the Lauterbach terrain were computed using the SRF method. This same vehicle was then run on NRMM and results were compared.

Vehicle Bundle Representatives (Tracked). Speed reduction matrices have been developed for the WARSIM SWA playbox using the M1A1 as a representative of the "high mobility tracked" bundle, the M113A2 as a representative of the "medium mobility tracked" bundle, and the D7 Dozer as a representative of the "low mobility tracked" bundle. Waterways Experiment Station (WES) concurs that these vehicles are reasonable representatives of the mobility bundles.

NRMM Results (Tracked). The representative vehicles were run on the NATO Reference Mobility Model (NRMM), simulating terrain in the Lauterbach region of Germany, the Al Mafraq region of Jordan, the Cheorweon region of South Korea, and the Dasht E Arzhan region of Iran. Standard NRMM results of these simulations are presented in Figures A1 through A4. The figures are plots of cumulative average speed as a function of percent of total area and are often referred to as speed distributions or speed profiles. They offer a convenient 


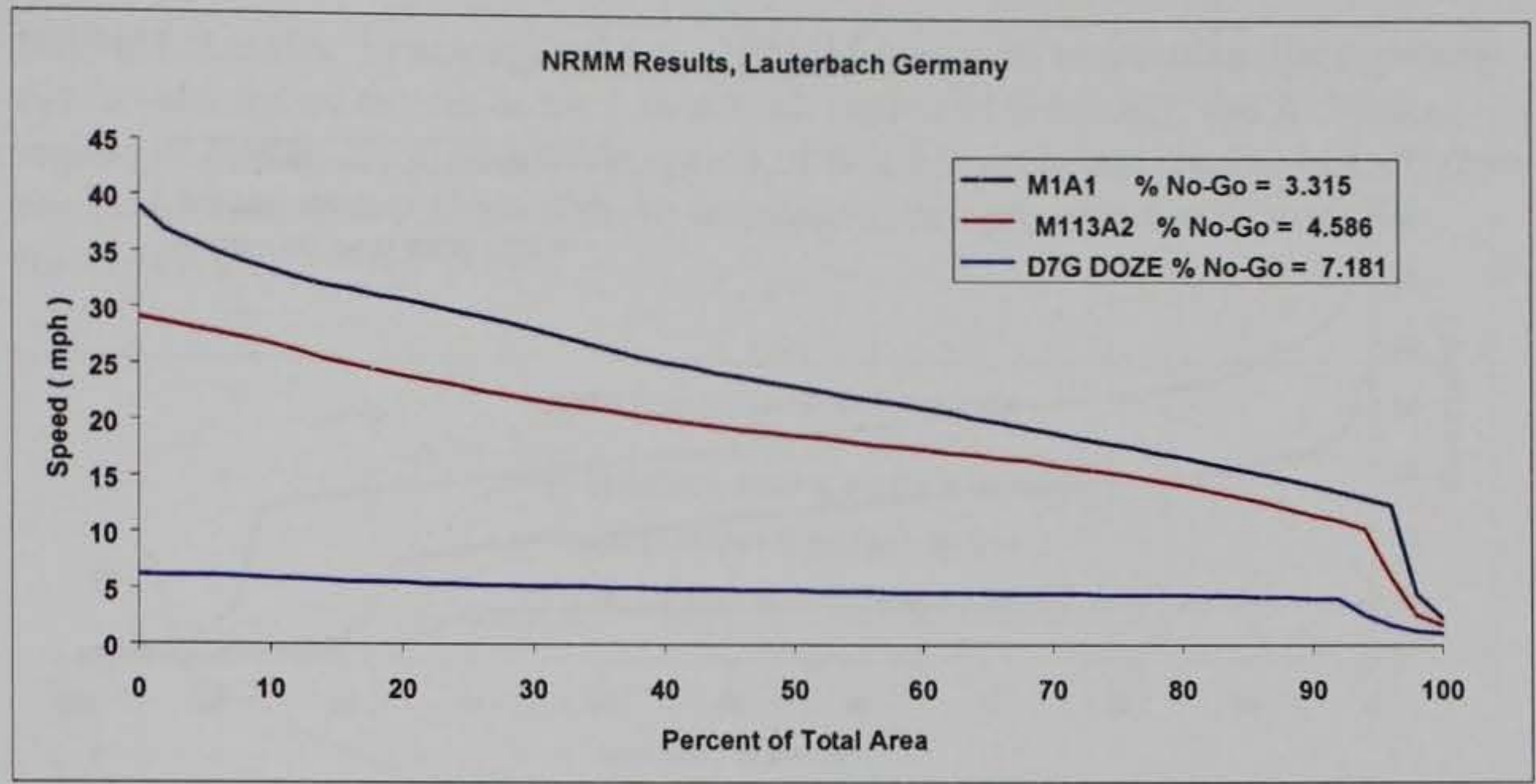

Figure A1. Lauterbach Germany, tracked representative vehicles

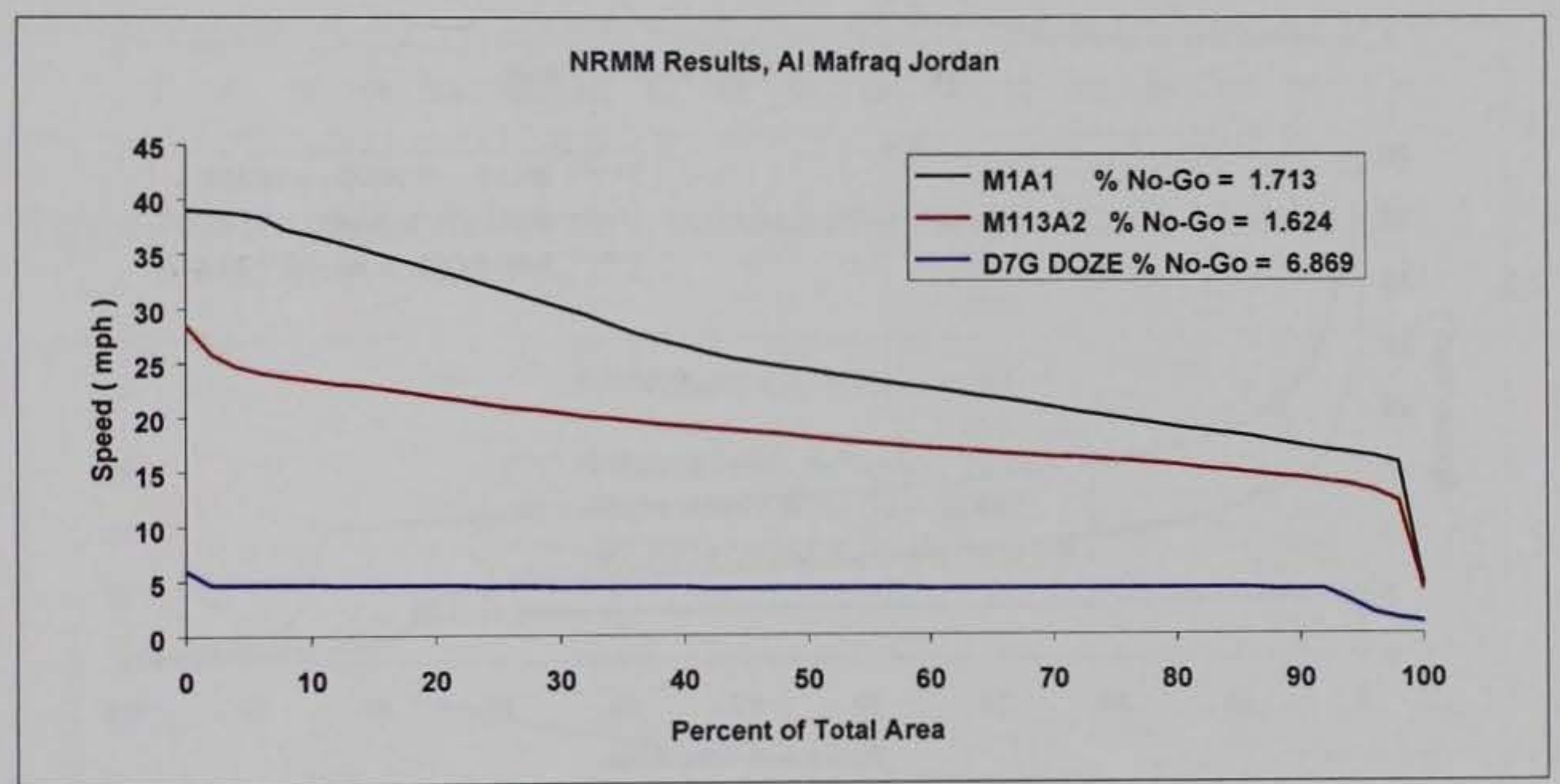

Figure A2. Al Mafraq Jordan, tracked representative vehicles

means of comparing the performance of several vehicles over the thousands of units that make up a single terrain data set.

The D7 shows little speed variation on a given terrain. This is because it is geared for low speed, high tractive force operation. It's maximum speed is less than $10 \mathrm{mph}$. Percent of no-go terrain varies noticeably on the different areas however. Overall average speed differences between the M1A1 and the M113A3 are about 20 percent on the Lauterbach terrain, 28 percent on the Jordanian terrain, and about 24 percent on the South Korean and Iranian terrain. There is a large speed difference between the D7 and the other vehicles on the Lauterbach terrain. This is caused by a low top speed, typical of construction and engineer equipment. On the more severe areas (Korea and Iran), the difference between the D7 and the other vehicles is not as large. The high mobility representative is significantly faster and has fewer no-gos on all terrain simulated than the medium 


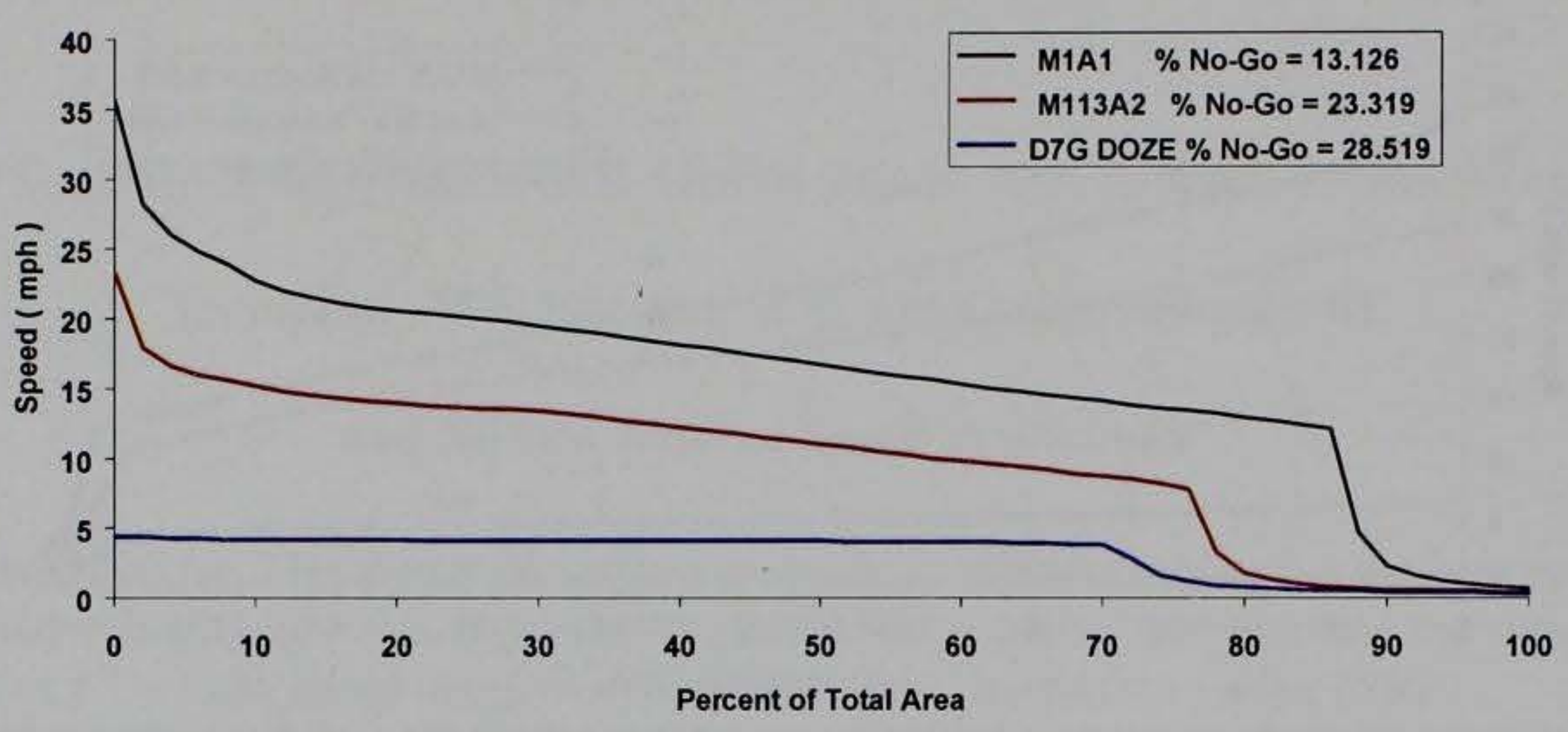

Figure A3. Dasht E Arzhan Iran, tracked representative vehicles

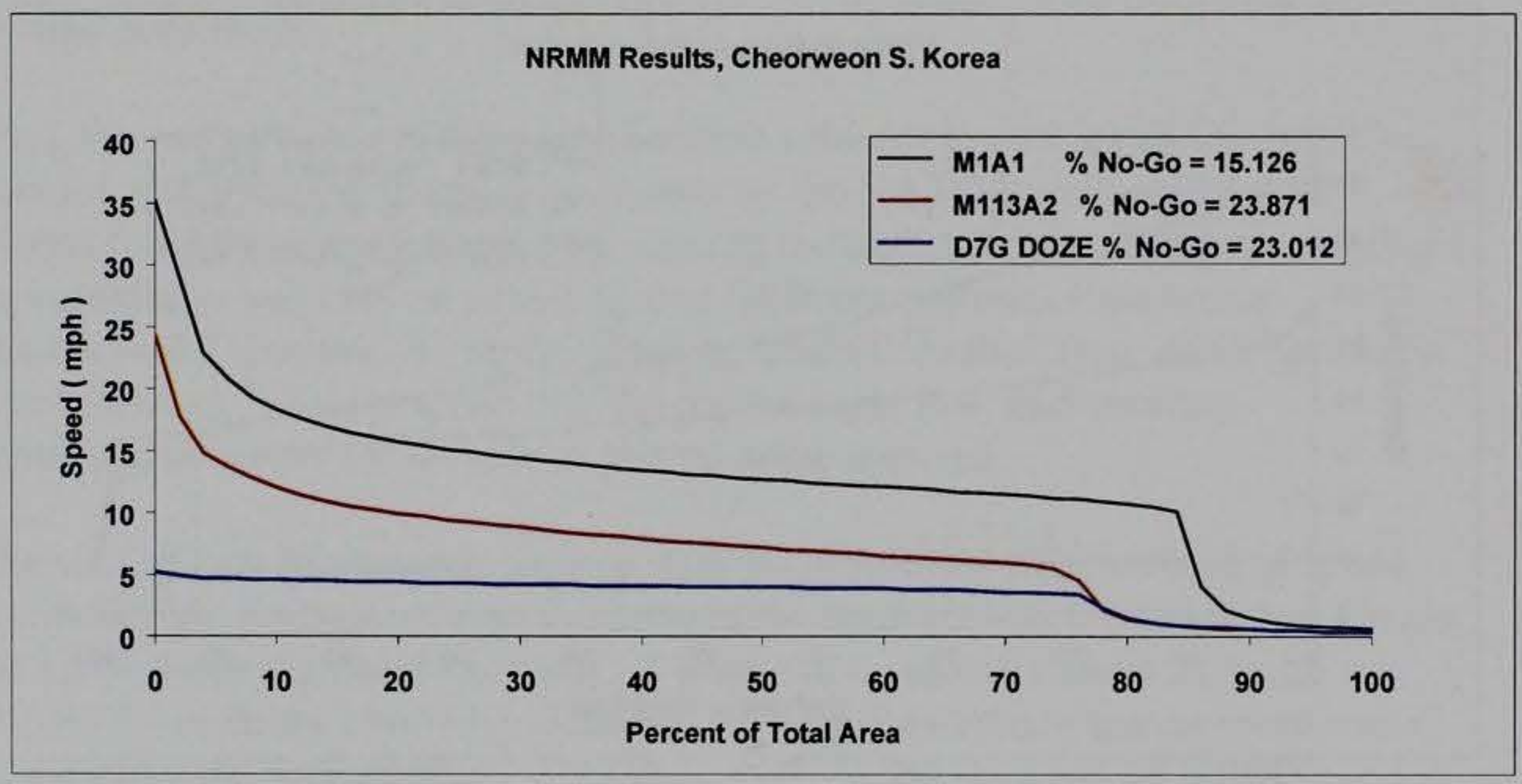

Figure A4. Cheorweon, South Korea, tracked representative vehicles

mobility representative. The medium mobility representative is significantly faster and also has fewer no-gos than the low mobility representative on all terrain simulated.

Vehicle Bundle Representatives, Tractor/Trailers. Speed reduction matrices have been developed for the WARSIM SWA playbox using the M1025A2 towing the High Mobility Trailer (HMT) as a representative of the "high mobility tractor/ trailer" bundle, the M923 towing the M1061 trailer as a representative of the "medium mobility tractor/trailer" bundle, and the M911 towing the M747 trailer as a representative of the "low mobility tractor/trailer" bundle. WES concurs that these vehicles are reasonable representatives of the mobility bundles. 
NRMM Results, Tractor/Trailers. NRMM was used to simulate the representative vehicles on terrain in the Lauterbach region of Germany, the Al Mafraq region of Jordan, the Cheorweon region of South Korea, and the Dasht E Arzhan region of Iran. The results of these simulations are presented in Figures A5 through A8.

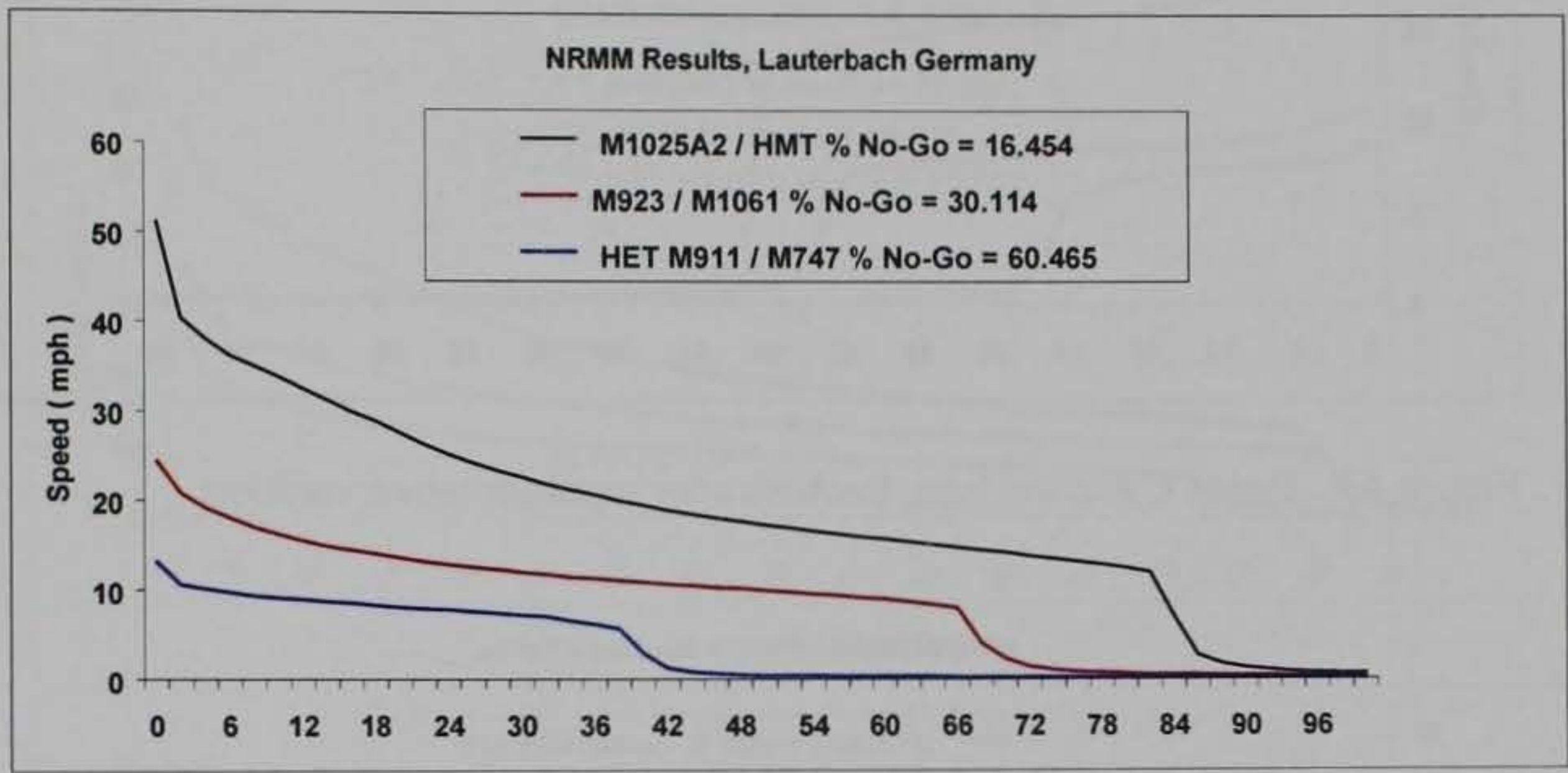

Figure A5. Lauterbach Germany, tractor/trailer representative vehicles

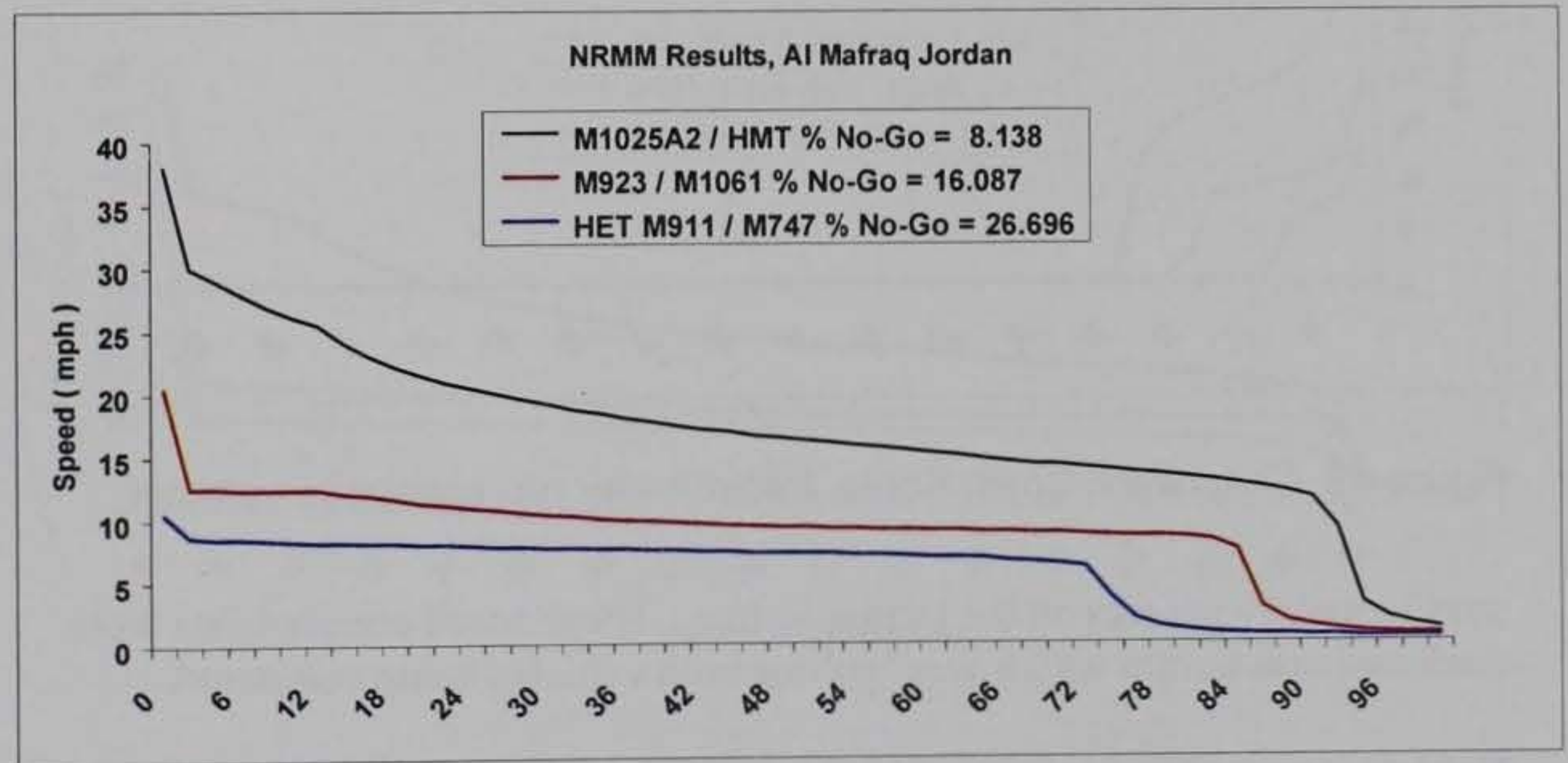

Figure A6. Al Mafraq Jordan, tractor/trailer representative vehicles

The high mobility representative is significantly faster and has significantly fewer no-gos on all terrain simulated than the medium mobility representative. The medium mobility representative is significantly faster and also has significantly fewer no-gos than the low mobility representative on all terrain simulated. Overall average speed differences between the M1025/HMT and the M923/M061 are about 47 percent on the Lauterbach terrain, 38 percent on the Jordan and Korean terrain, and 29 percent on the terrain in Iran. Overall average speed differences between the M923/M1061 and the M911/M747 are about 42 percent on the Lauterbach terrain, 30 percent on the Jordan and Korean 


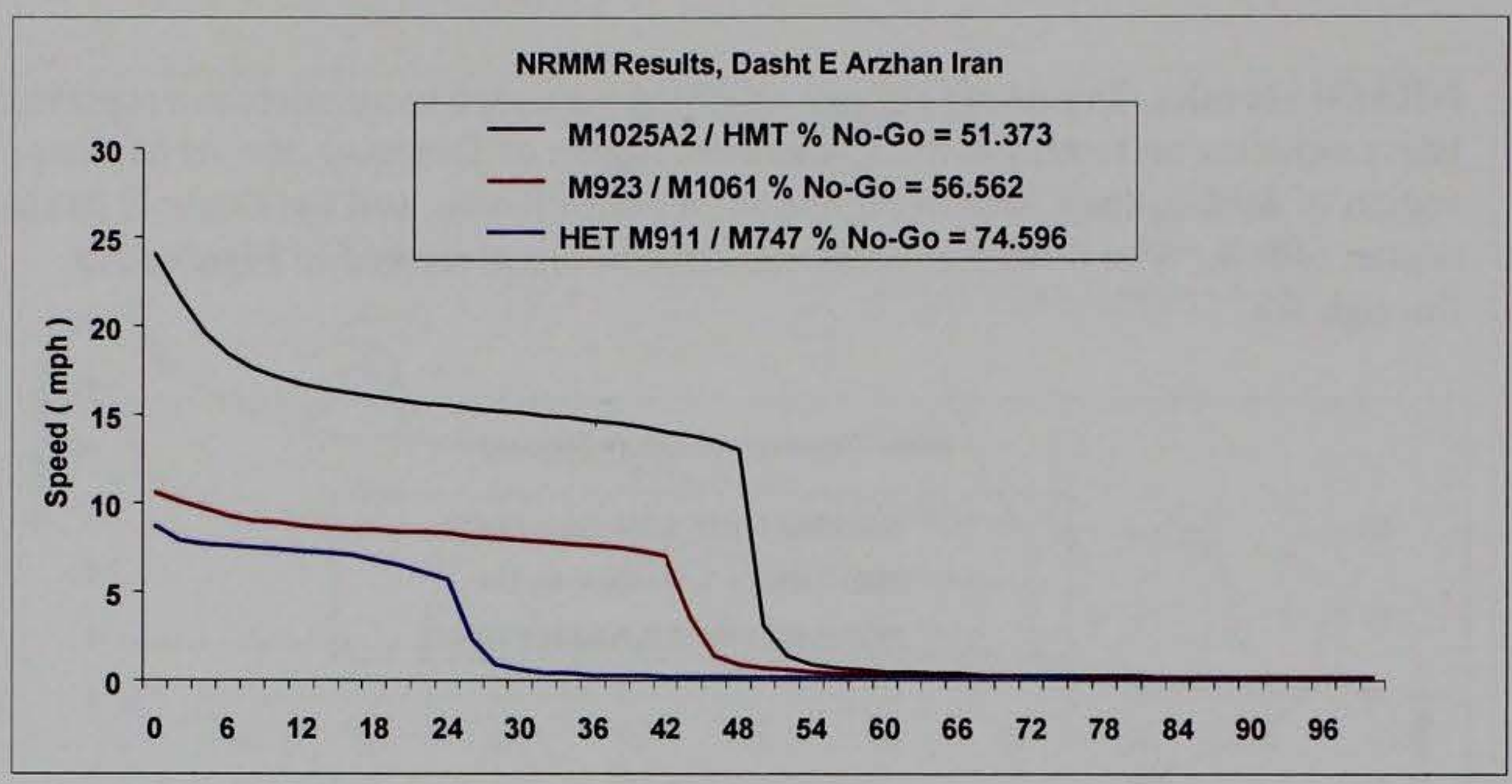

Figure A7. Dasht E Arzhan Iran, tractor/trailer representative vehicles

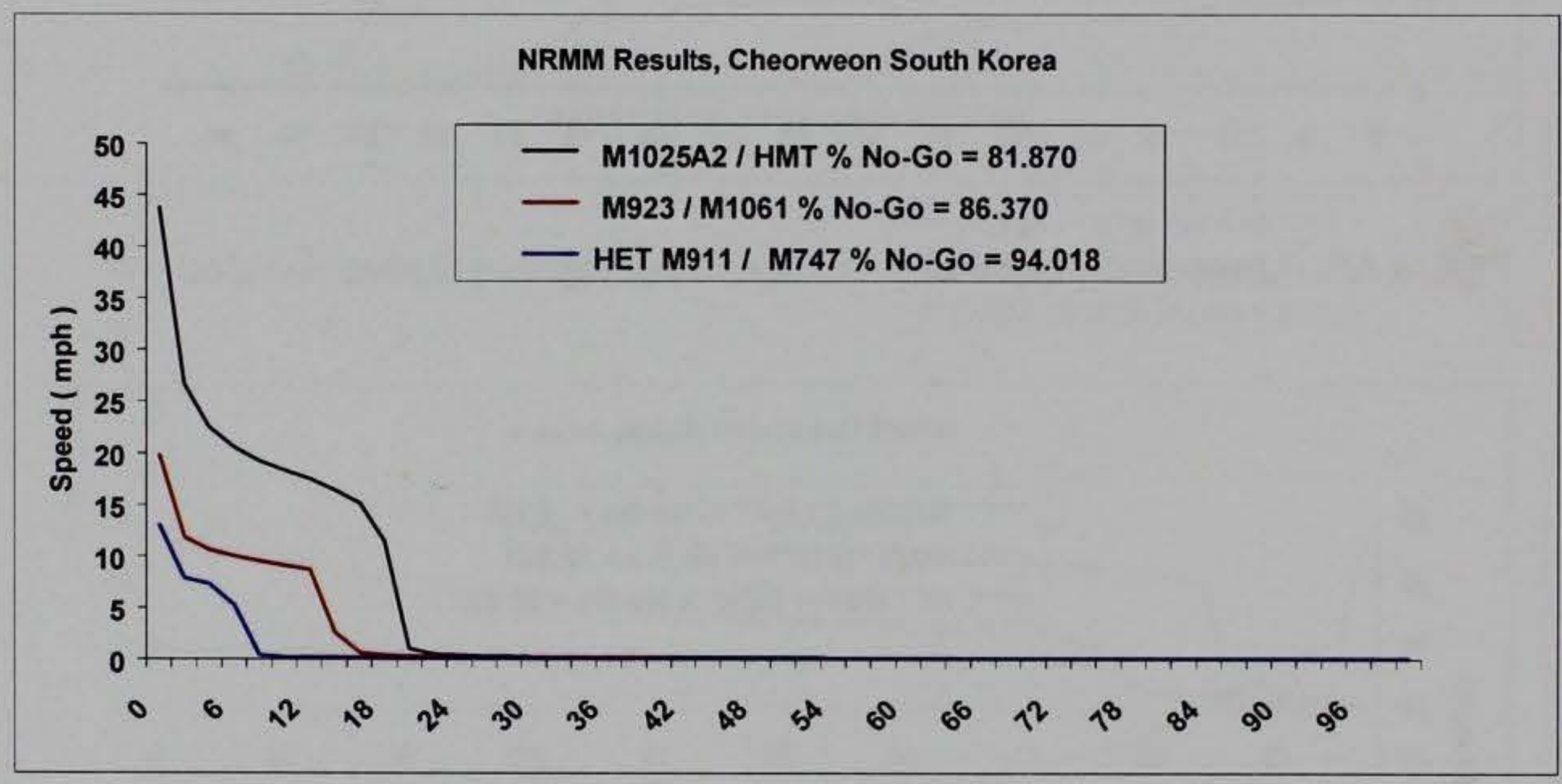

Figure A8. Cheorweon South Korea, tractor/trailer representative vehicles

terrain, and 19 percent on the terrain in Iran. These speed comparisons were made only on terrain which was 'go' for both vehicles being compared.

Vehicle Bundle Representatives, Wheeled. Speed reduction matrices have been developed for the WARSIM SWA playbox using the M1025A2 as a representative of the "high mobility wheeled" bundle, the M923 as a representative of the "medium mobility wheeled" bundle, and the M911 tractor as a representative of the "low mobility wheeled" bundle. WES concurs that these vehicles are reasonable representatives of the mobility bundles. The M911 is not classified as a low mobility wheeled vehicle in the WARSIM bundling document, it is being used as a surrogate vehicle to represent this category. The reason for this is that neither WES nor AMSAA have sufficient data to create an NRMM data file for any of the vehicles classified as "low mobility wheeled" in the bundling document. 
NRMM Results, Wheeled. NRMM was used to simulate the representative vehicles on terrain in the Lauterbach region of Germany, the Al Mafraq region of Jordan, the Cheorweon region of South Korea, and the Dasht E Arzhan region of Iran. The results of these simulations are presented in Figures A9 through A12.

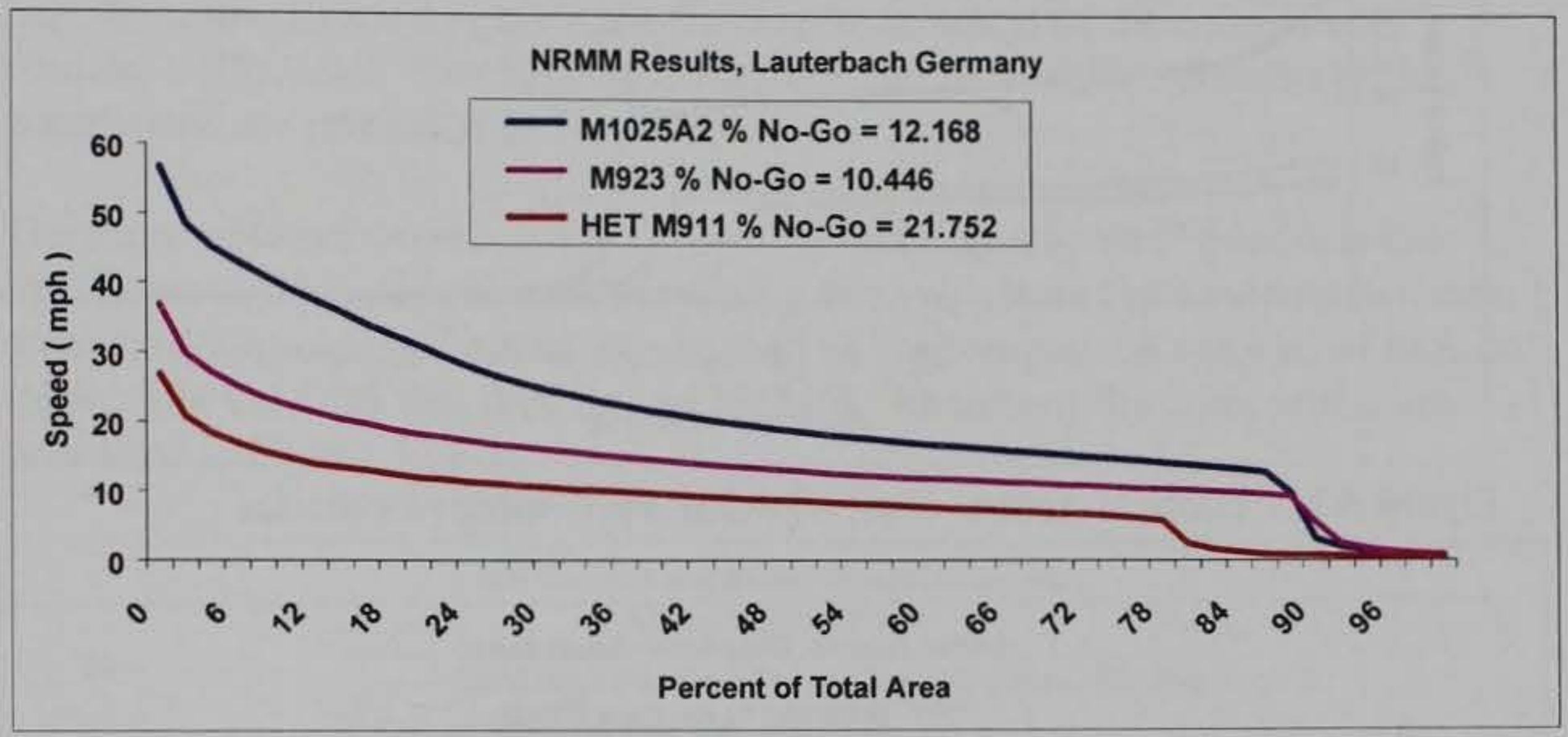

Figure A9. Lauterbach Germany, wheeled representative vehicles

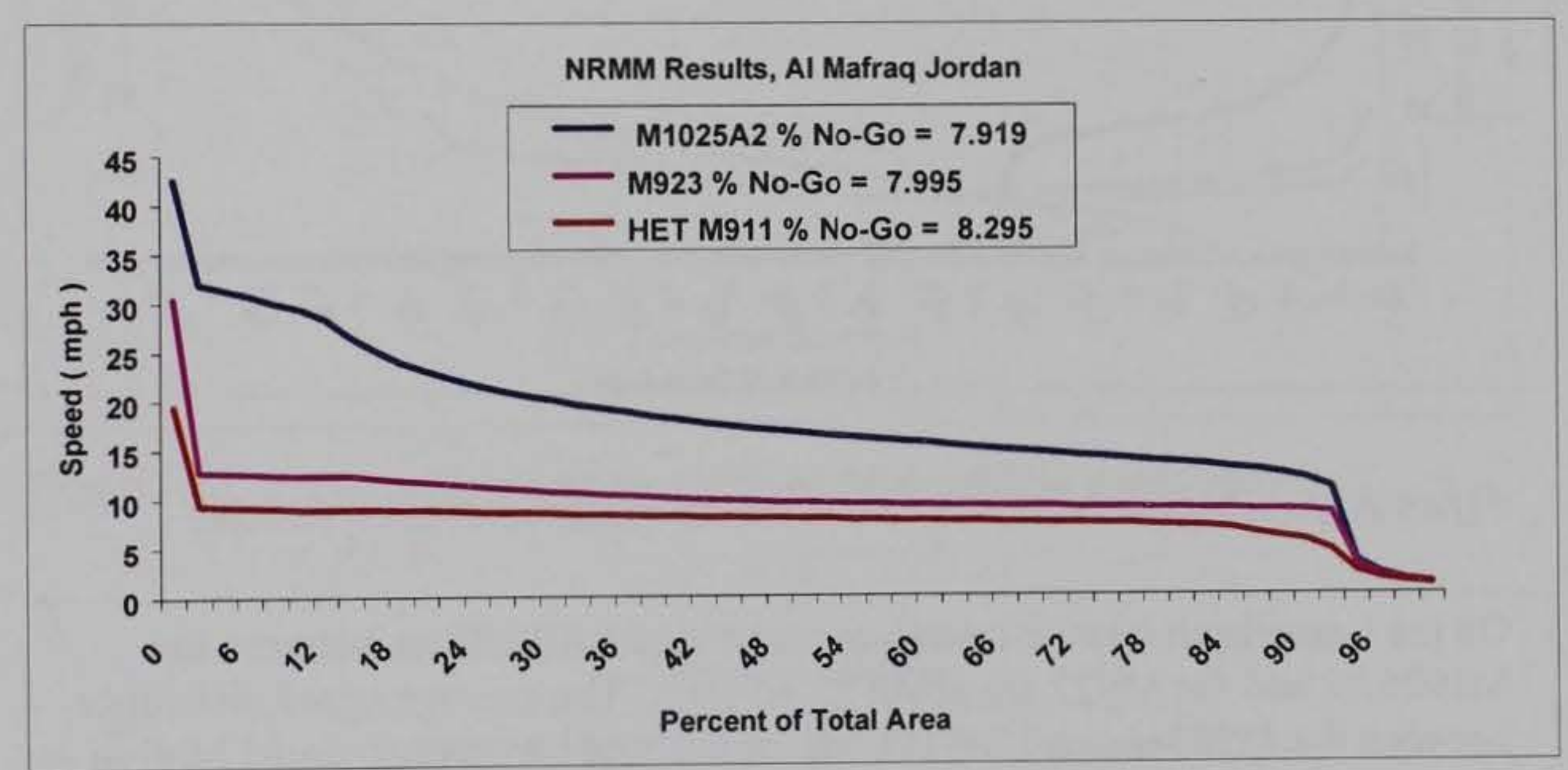

Figure A10. Al Mafraq Jordan, wheeled representative vehicles 


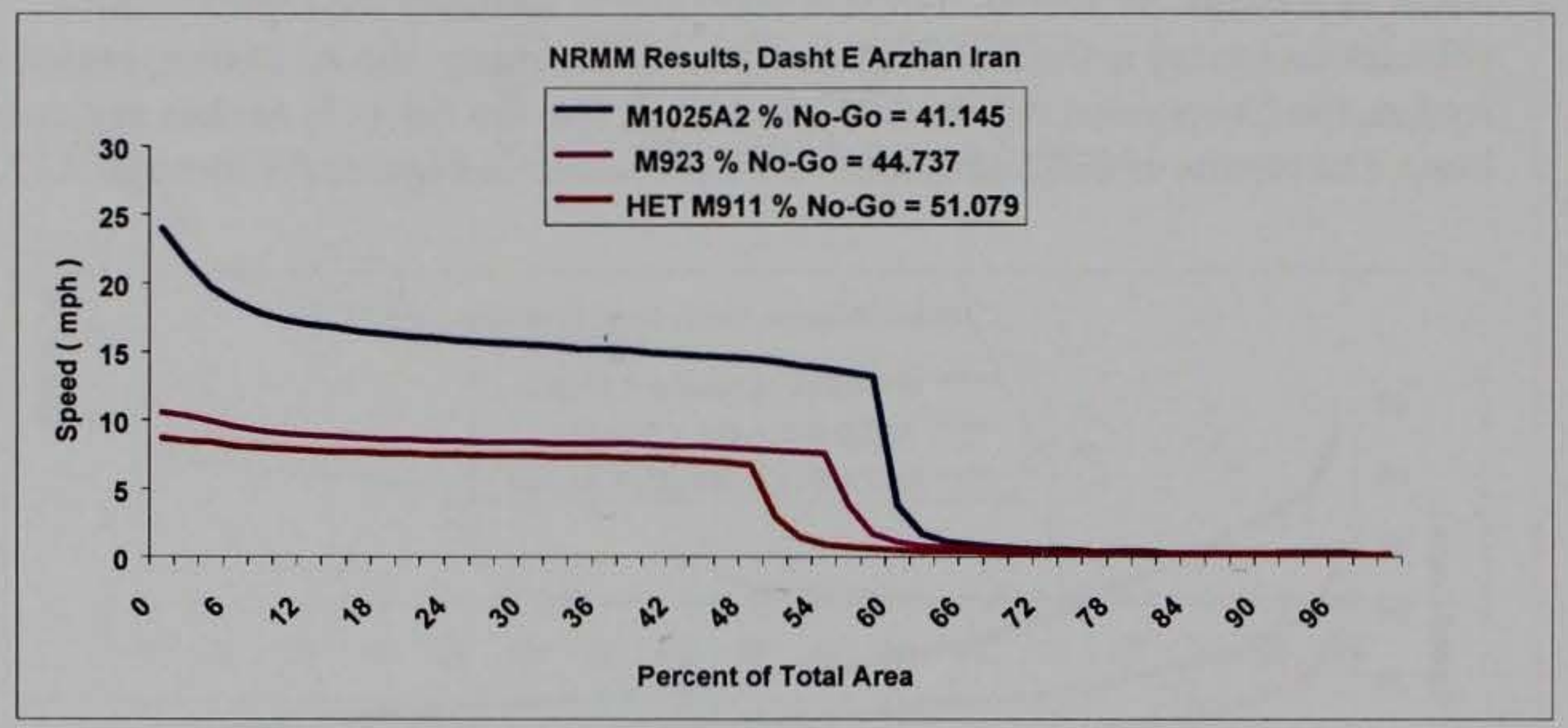

Figure A11. Dasht E Arzhan Iran, wheeled representative vehicles

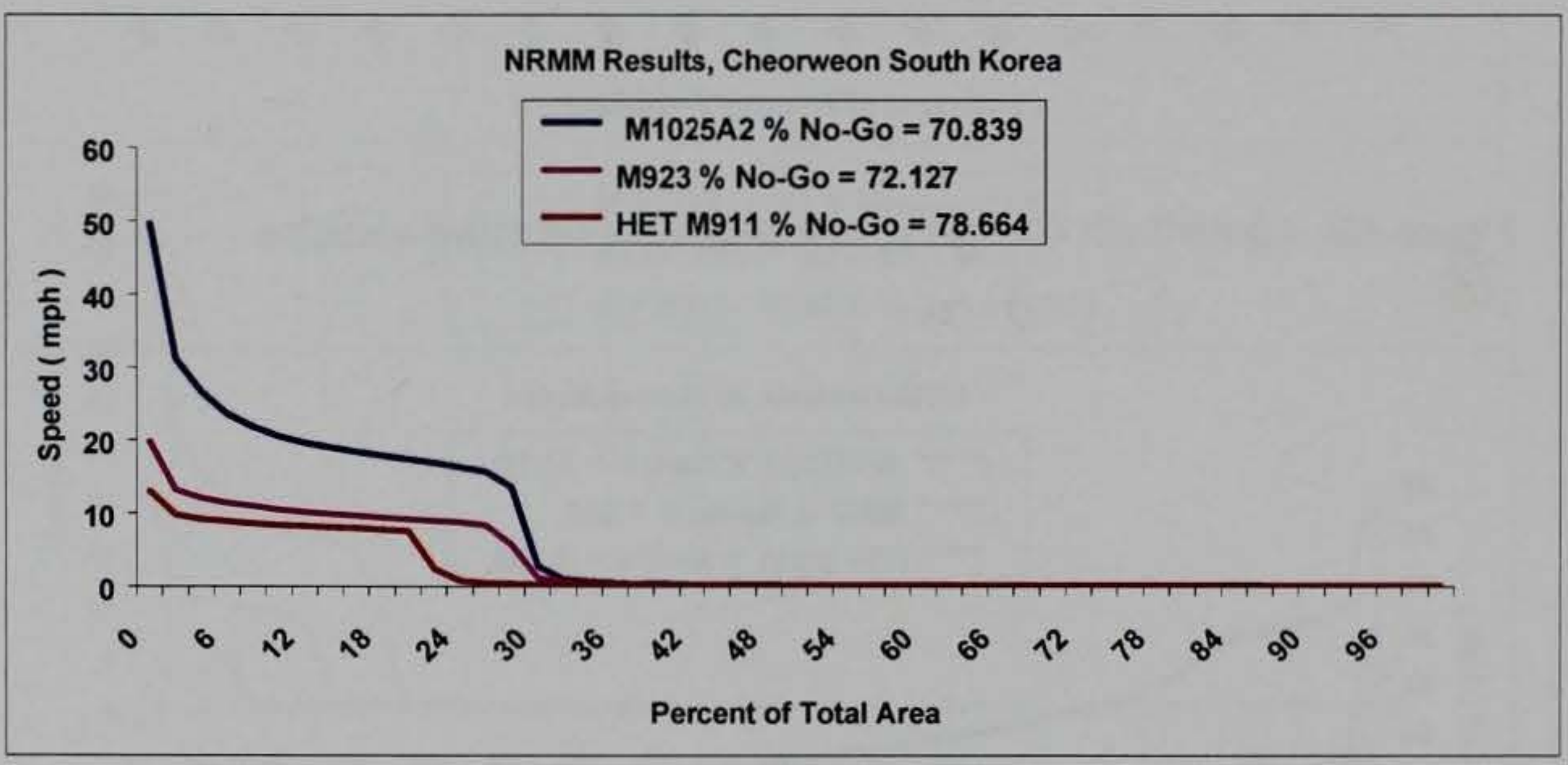

Figure A12. Cheorweon South Korea, wheeled representative vehicles

On the Lauterbach terrain, overall average speed differences between the M1025A2 and the M923 are about 35 percent. The average speed difference between the M923 and the M9111 tractor is about 30 percent. In Al Mafraq Jordan, the differences are about 45 percent between the M1025A2 and the M923, and about 24 percent between the M923 and the M911 tractor. On the Dasht E Arzhan terrain speed differences are 50 percent between the M1025A2 and the M923, and 12 percent between the M923 and the M911 tractor, the M911 also has significantly more no-gos than does the M923. In the South Korean terrain speed differences are about 50 percent between the M1025A2 and 17 percent between the M923 and M911 tractor, the M911 has significantly more no-gos than the other two vehicles on this terrain.

Comparison of SRF Generated and NRMM Computed Speeds. SRF derived speeds and NRMM computed speeds for vehicles chosen from the mobility bundles were compared. The comparison was done using speed reduction matrices developed for the bundle representative vehicles in the Lauterbach region of Germany. The values plotted are SRF and NRMM computed speeds for each unit of 
the Lauterbach terrain data file. The speeds were sorted from highest to lowest. All terrain units were weighted equally in the percent of total area computation.

The representative vehicle for the "high mobility wheeled" bundle is the M1025A2 HMMWV. SRFs resulting from running this vehicle on the Lauterbach terrain were used to multiply the maximum speed of the comparison vehicle, a BRDM-2. The BRDM-2 was then run on NRMM. Results of the comparison are presented in Figure A13.

The representative vehicle for the "medium mobility wheeled" bundle is the M923 truck. SRFs resulting from running this vehicle on the Lauterbach terrain were used to multiply the maximum speed of the comparison vehicle, a GAZ-66 truck. The GAZ-66 was then run on NRMM. Results of the comparison are presented in Figure A14.

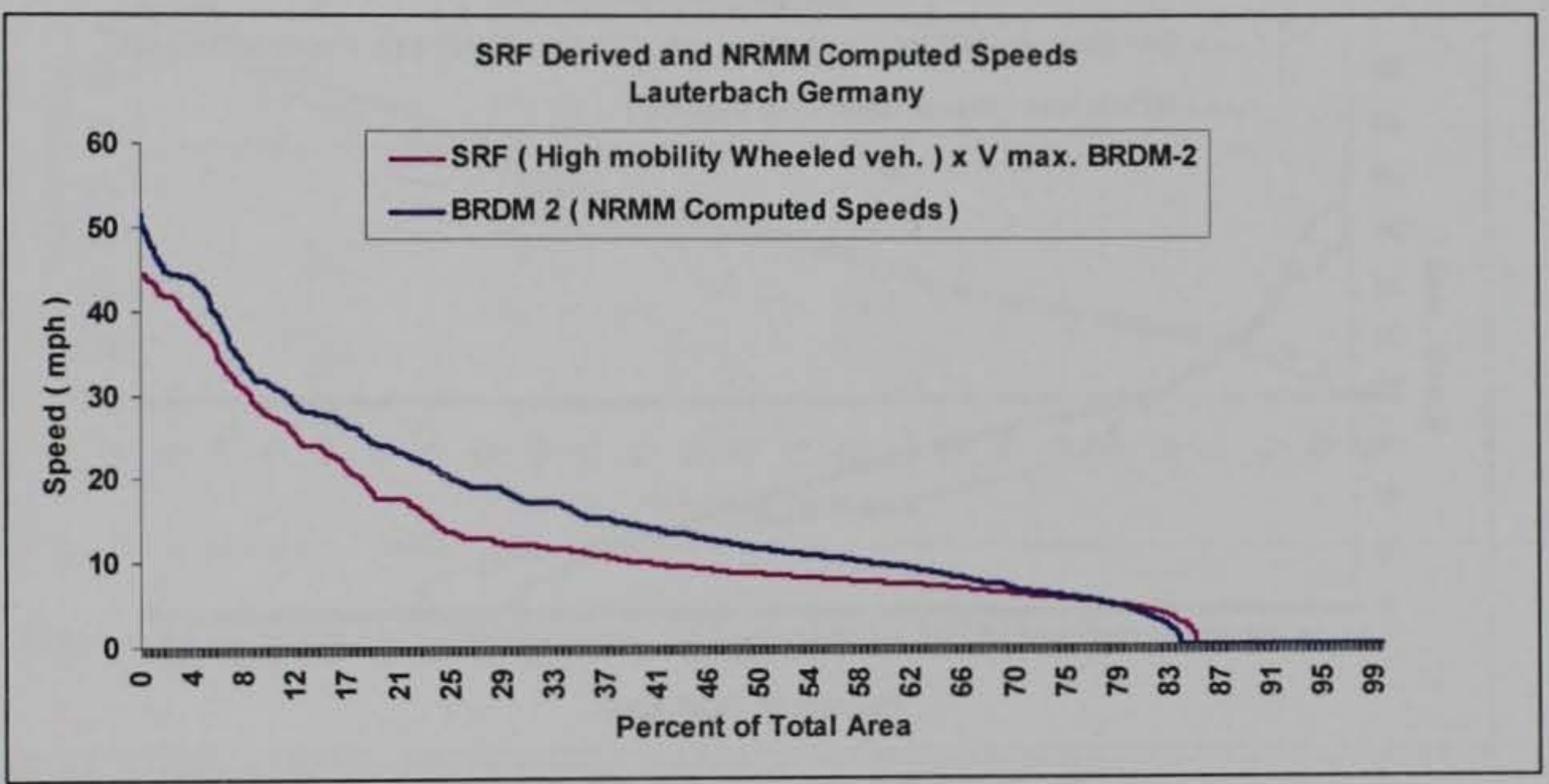

Figure A13. High mobility wheeled vehicle SRF, NRMM comparison

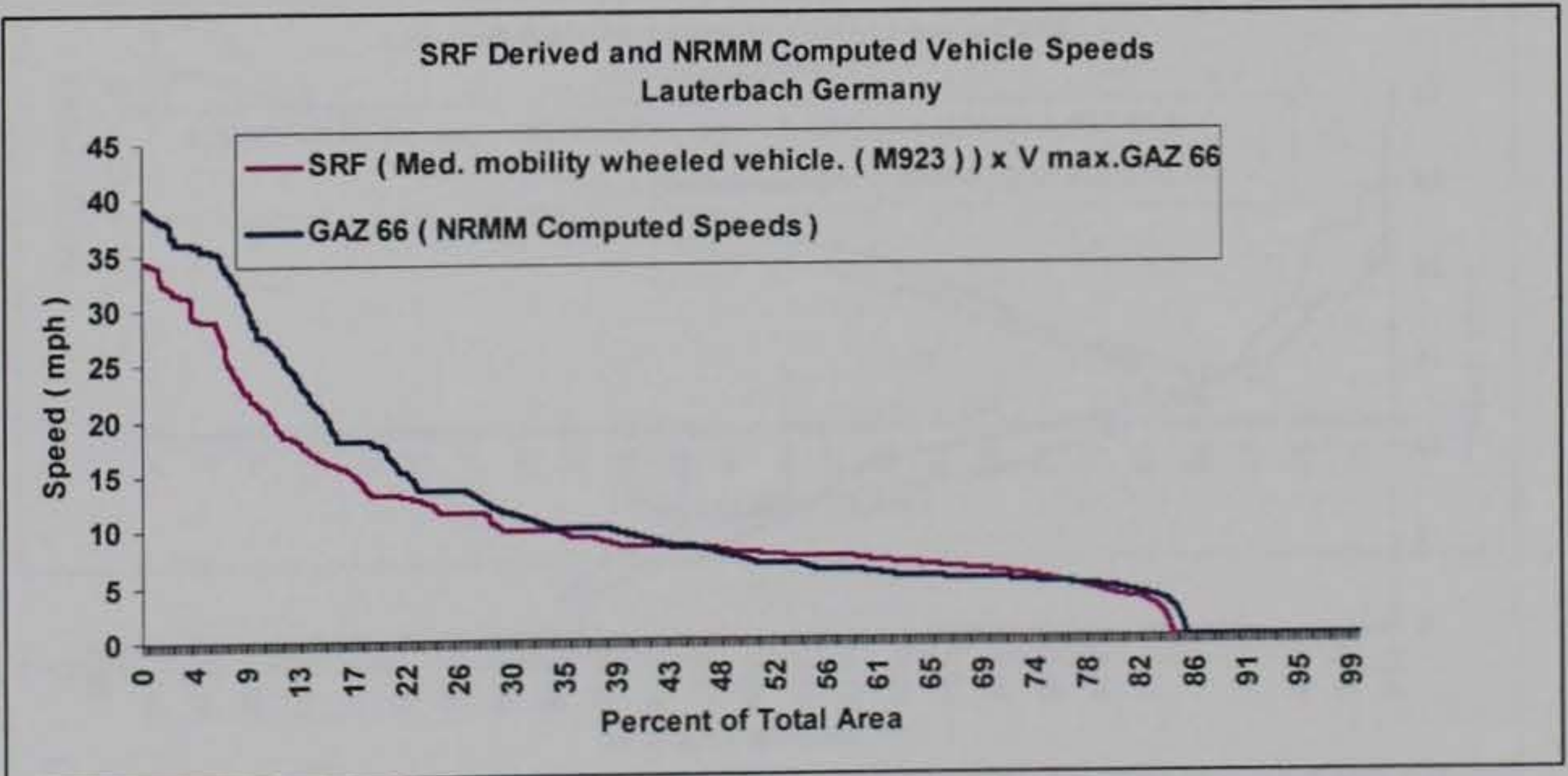

Figure A14. Medium mobility wheeled vehicle SRF, NRMM comparison 
The "high mobility tractor trailer" bundle representative vehicle is the M1025A2 towing a High Mobility Trailer (HMT). SRFs resulting from running this vehicle on the Lauterbach terrain were used to multiply the maximum speed of the comparison vehicle, an MTV towing an M1061 trailer (see conclusions for information on classification of MTV). The MTV/M1061 was then run on NRMM.

Results of the comparison are presented in Figure A15.

The "medium mobility tractor trailer" bundle representative vehicle is the M923 towing an M1061 trailer. SRFs resulting from running this vehicle on the Lauterbach terrain were used to multiply the maximum speed of the comparison vehicle, an M1075 towing an M1076 trailer (PLS). The M1075/M1076 was then run on NRMM. Results of the comparison are presented in Figure A16.

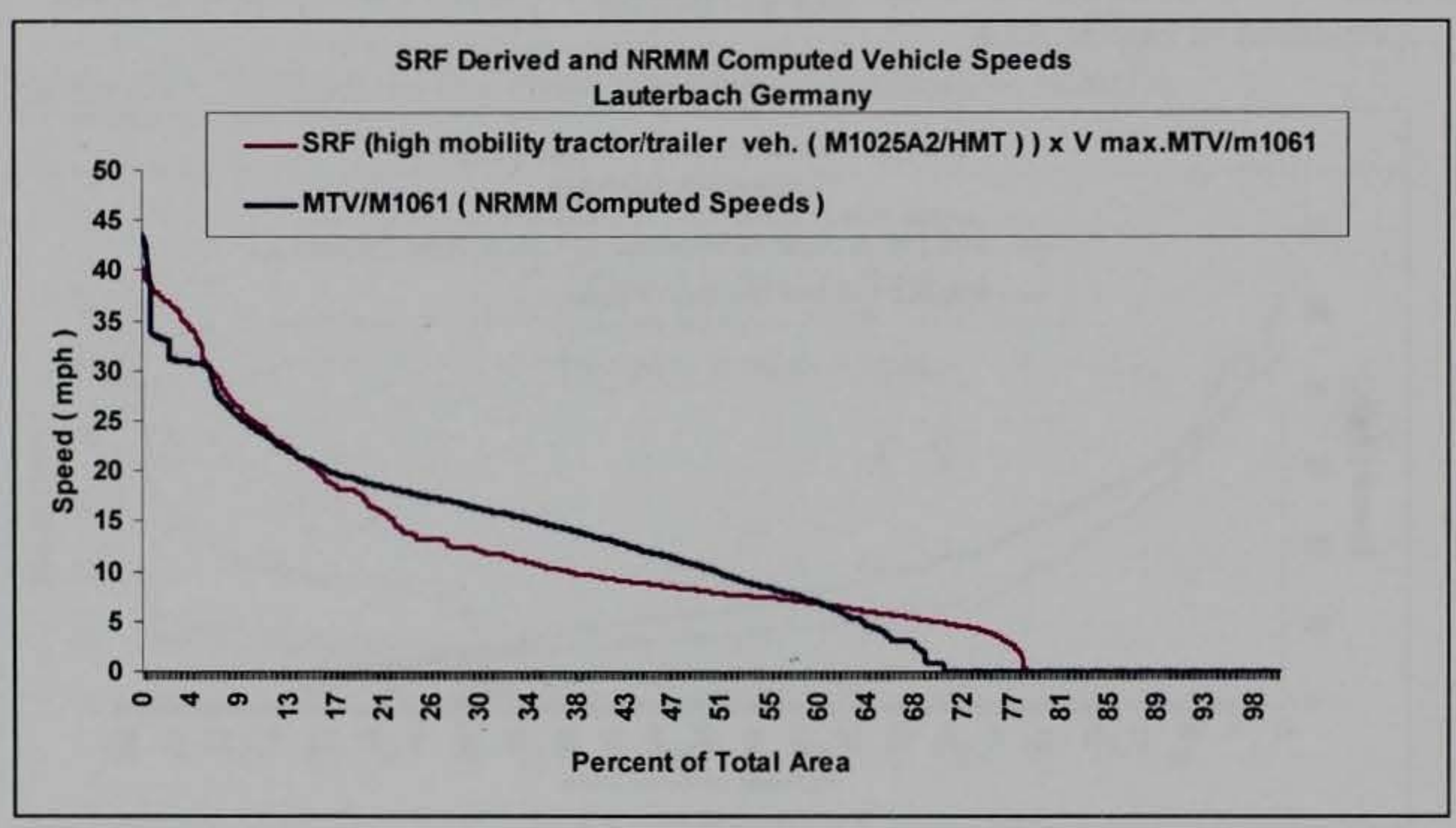

Figure A15. High mobility tractor/trailer SRF, NRMM comparison

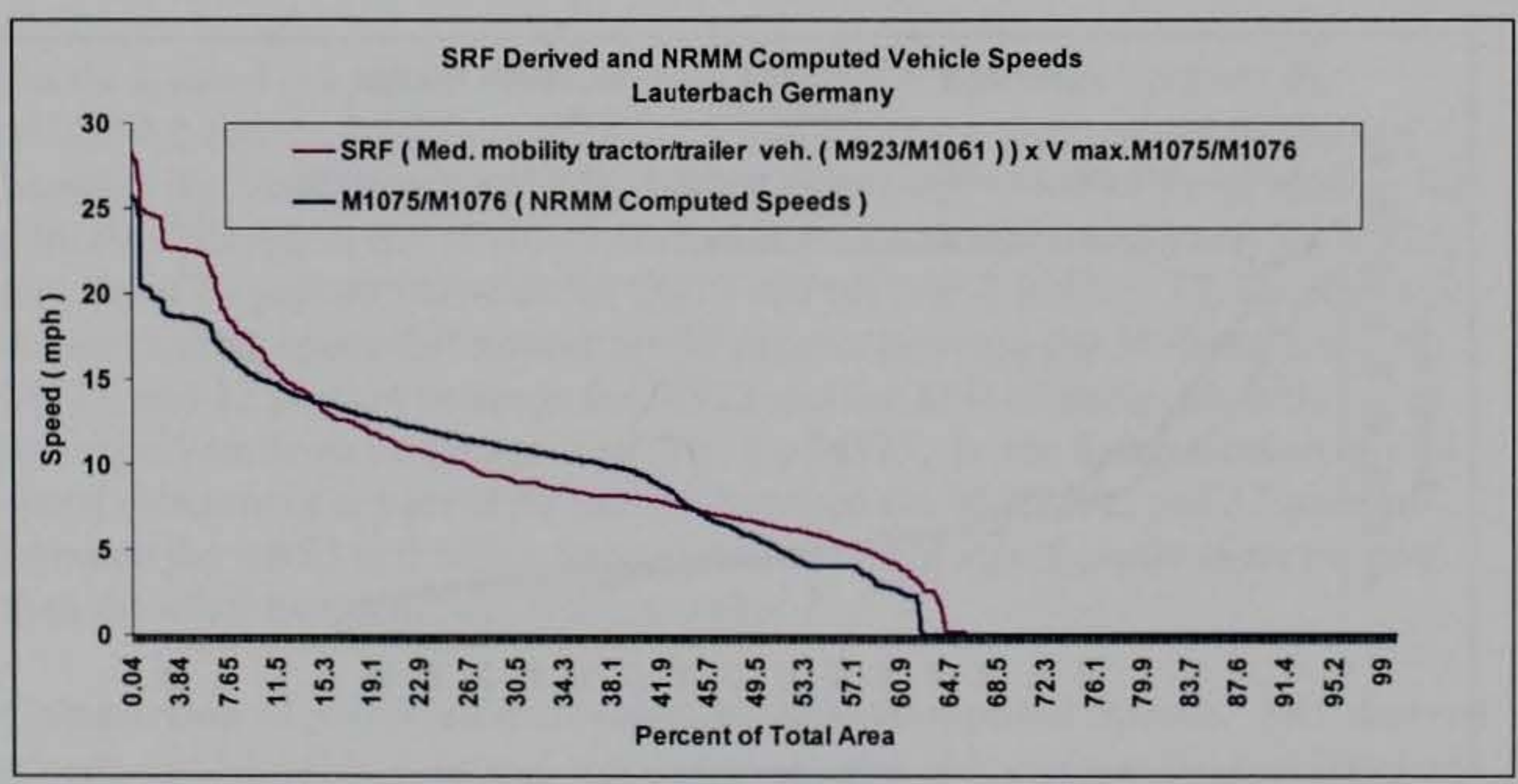

Figure A16. Medium mobility tractor/trailer SRF, NRMM comparison 
The representative vehicle for the "high mobility track" bundle is the M1A1. SRFs resulting from running this vehicle on the Lauterbach terrain were used to multiply the maximum speed of the comparison vehicle, a T-80 tank. The T-80 was then run on NRMM. Results of the comparison are presented in Figure A17.

The representative vehicle for the "medium mobility track" bundle is the M113A2. SRFs resulting from running this vehicle on the Lauterbach terrain were used to multiply the maximum speed of the comparison vehicle, an M60A3 tank. The M60A3 was then run on NRMM. Results of the comparison are presented in Figure A18.

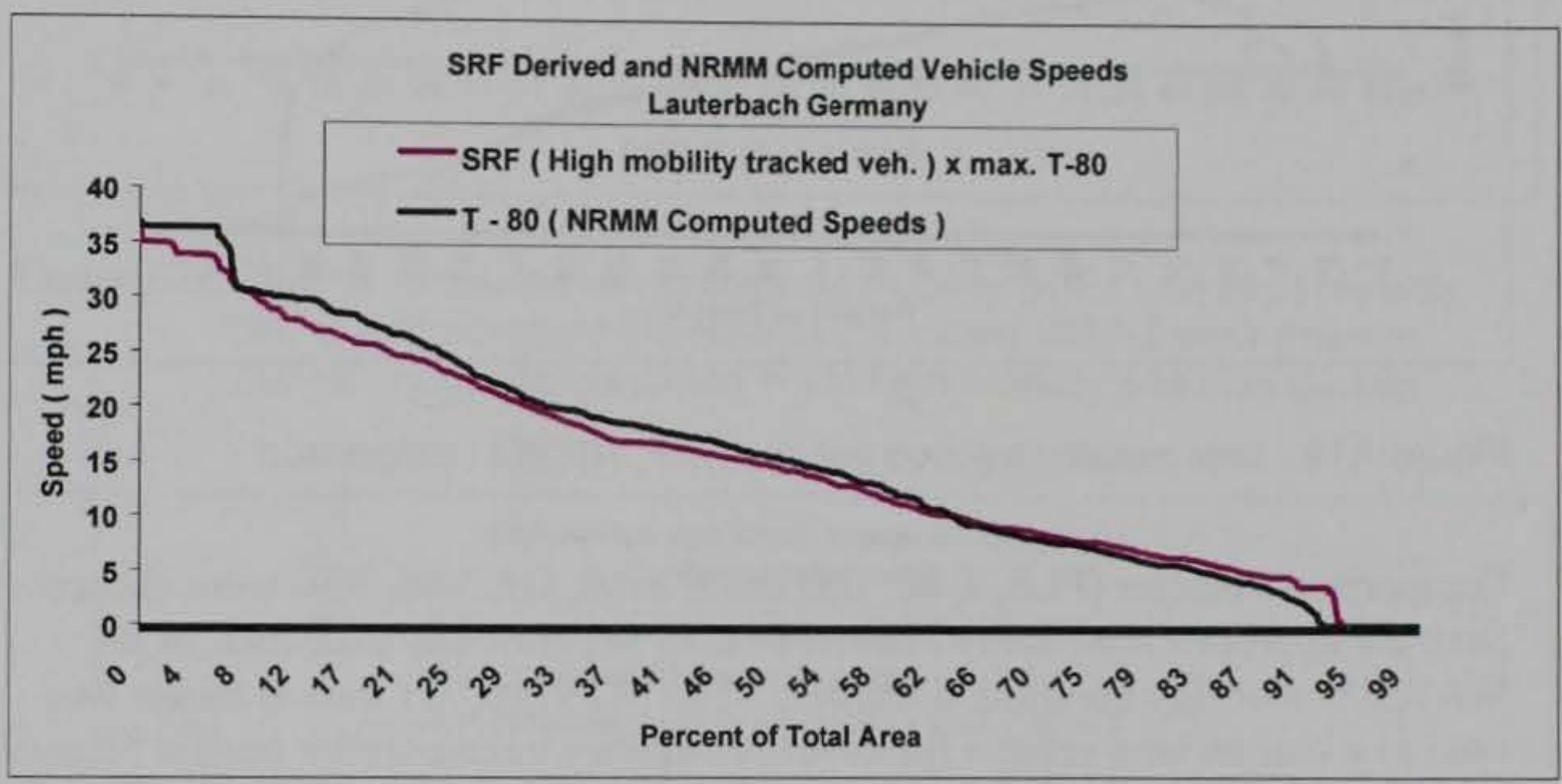

Figure A17. High mobility tracked vehicle SRF, NRMM comparison

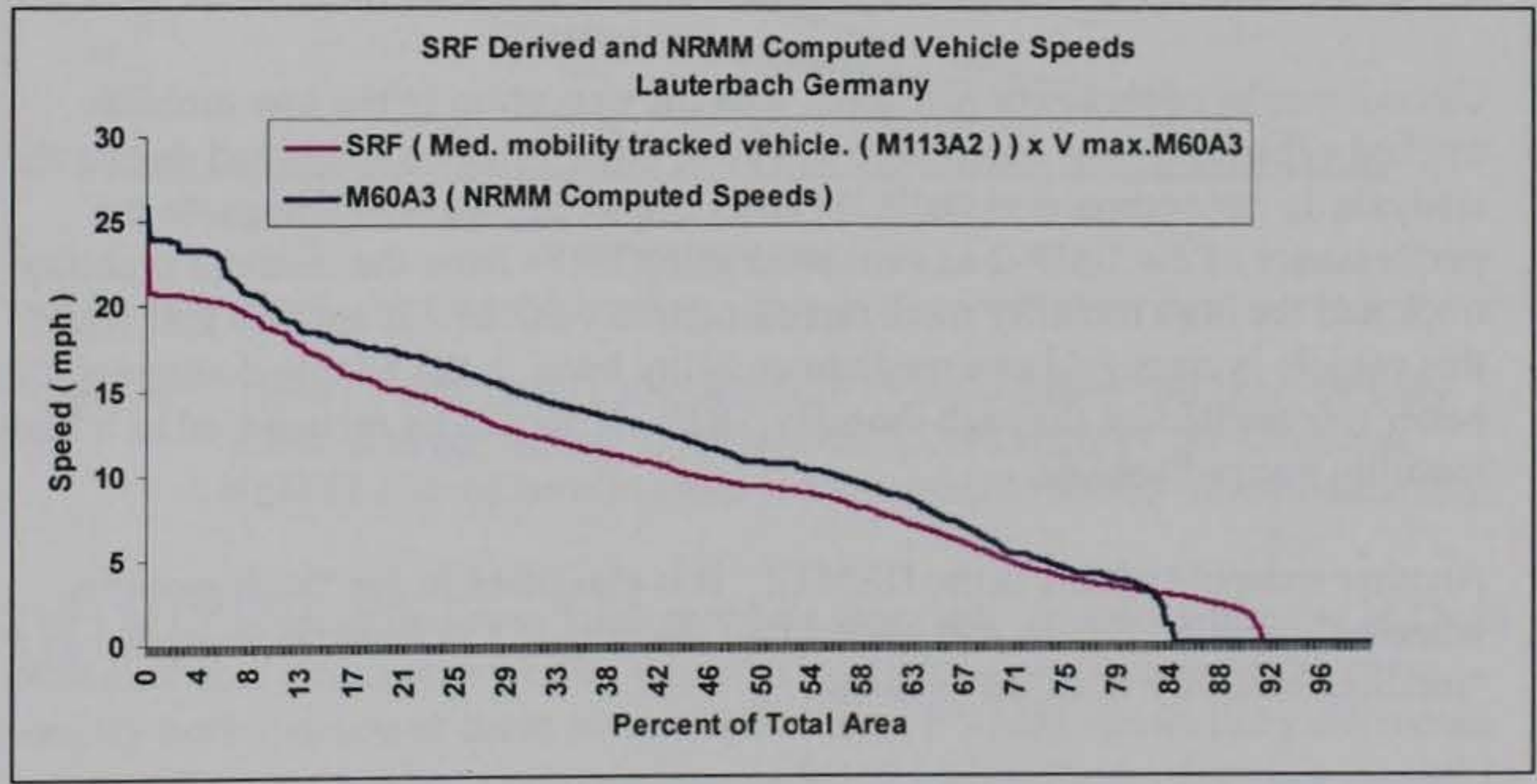

Figure A18. Medium mobility tracked vehicle SRF, NRMM comparison

The representative vehicle for the "low mobility track" bundle is the D7 Dozer. SRFs resulting from running this vehicle on the Lauterbach terrain were used to 
multiply the maximum speed of the comparison vehicle, an M9 ACE. The M9 was then run on NRMM. Results of the comparison are presented in Figure A19.

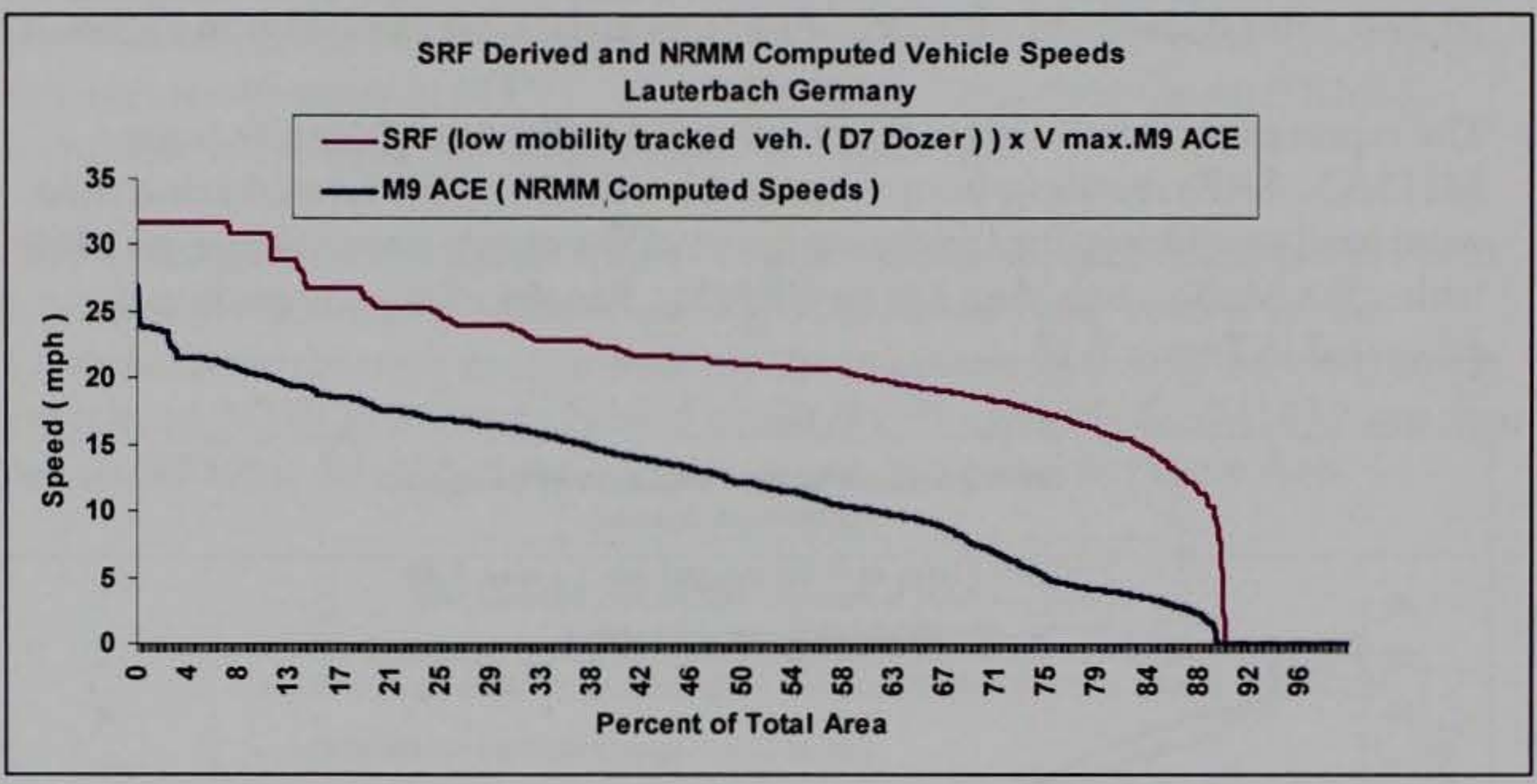

Figure A19. Low mobility tracked vehicle SRF, NRMM comparison

Comparison vehicles (PLS, T-80, BRDM-2, M60, GAZ-66, M9) were chosen from the appropriate mobility bundles as they are currently classified in the WARSIM mobility bundling document. The MTV/M1061 tractor-trailer was used as a comparison vehicle for the high mobility tractor-trailer bundle because further analysis (see conclusions) indicates that the MTV should be reclassified as a high mobility wheeled vehicle. A low mobility wheeled vehicle comparison was not done. This is because neither WES nor AMSAA have sufficient data on any of the vehicles in this bundle to create an NRMM data file.

Overall results of these comparisons, with the exception of the low mobility tracked vehicle, appear acceptable however, other vehicles examined during the analysis do not compare as well. As an example, Figure A20 compares the performance of the BMP-2 as simulated using SRFs from the medium mobility track and the high mobility track representative vehicles. It appears that while this vehicle is classified as a medium mobility track in the bundle document it is better represented by the high mobility SRFs. It should be reclassified as a high mobility tracked vehicle.

Another example of this is the HEMTT. It is classified in the "high mobility wheeled" bundle. Figure A21 shows that the HEMTT is better represented as a "medium mobility wheeled" vehicle. 


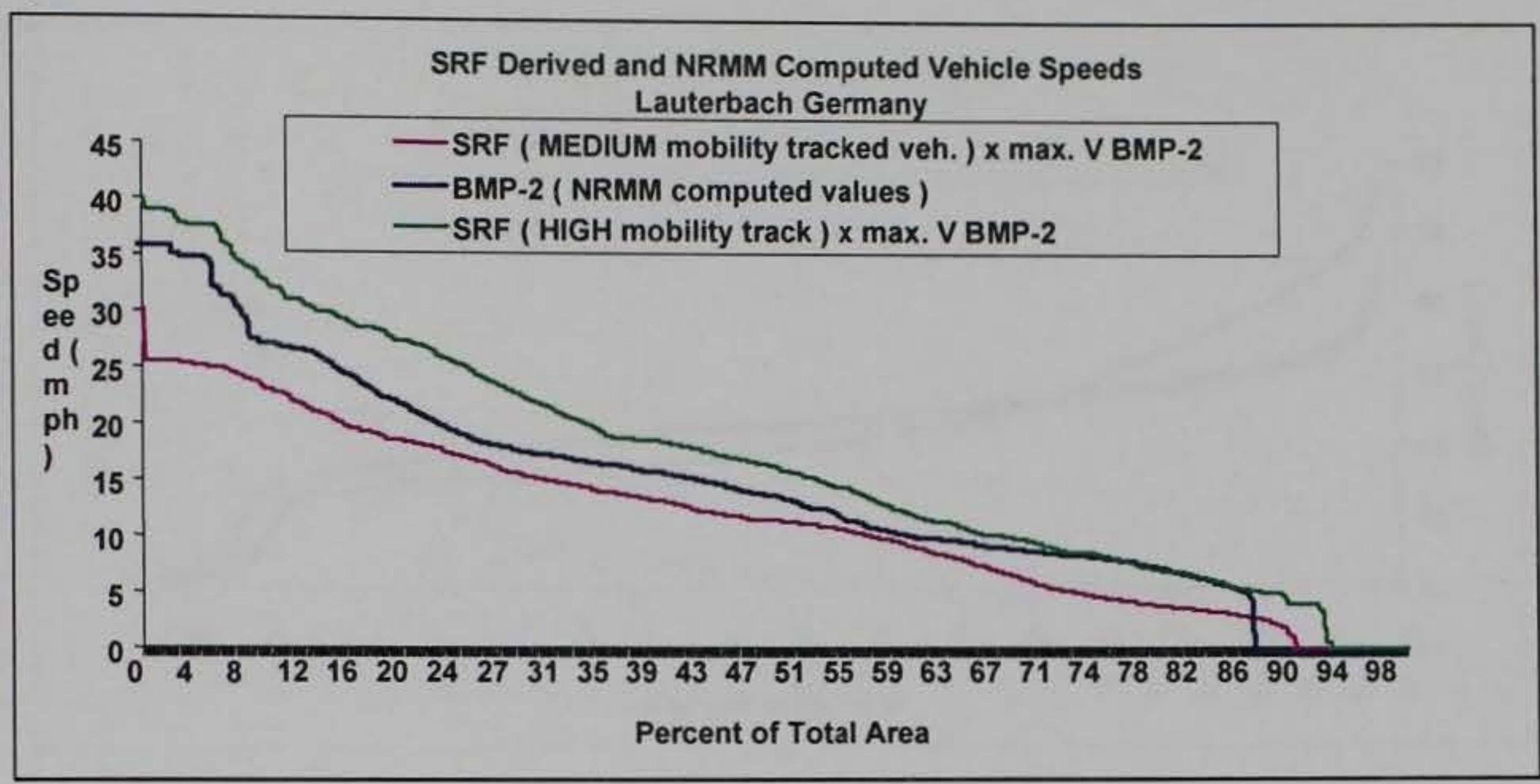

Figure A20. BMP-2 simulated using Medium mobility SRFs and High Mobility SRFs, and compared to NRMM computed BMP-2 performance. BMP-2 should be classified in the high mobility wheeled bundle

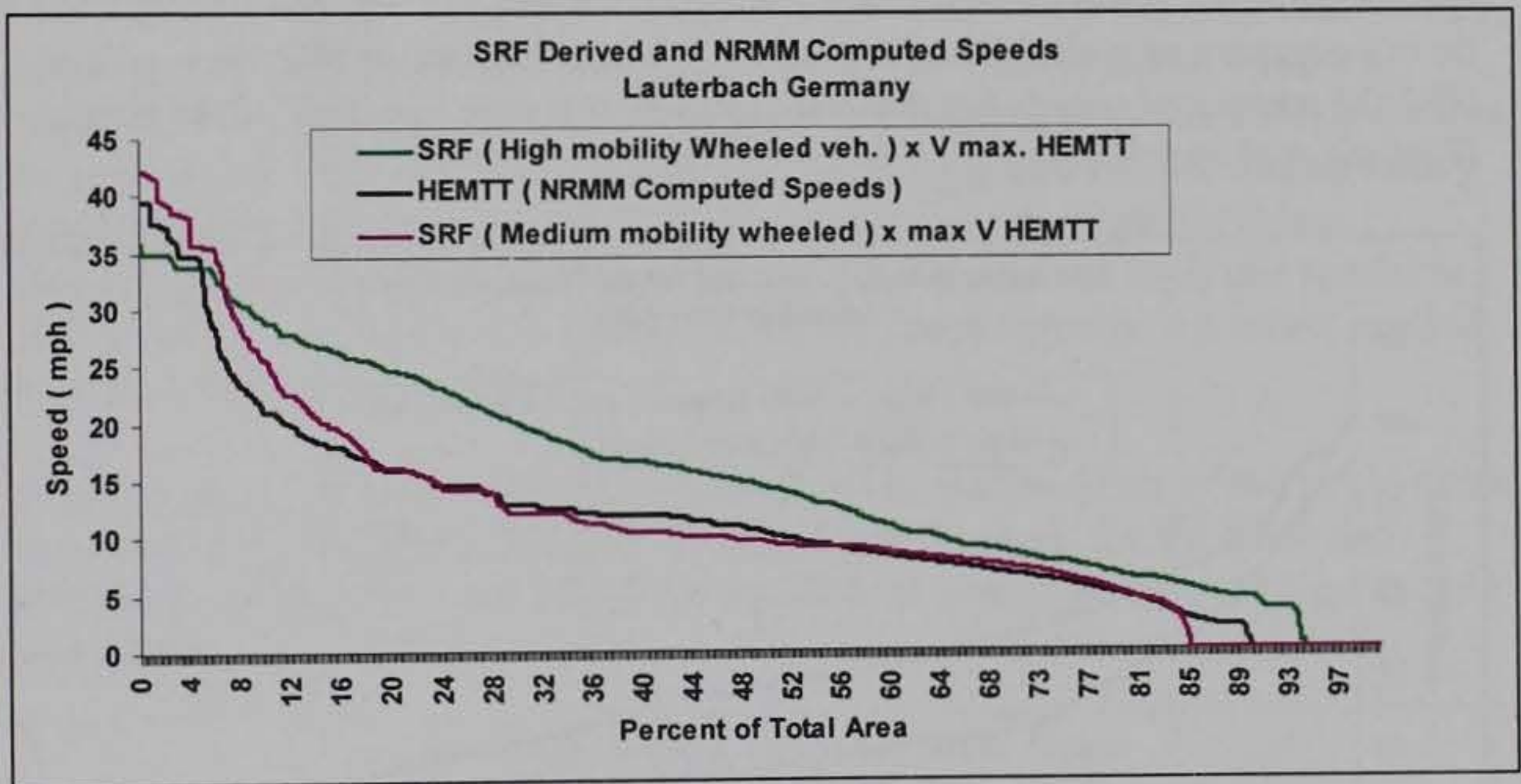

Figure A21. HEMTT simulated using Medium mobility SRFs and High Mobility SRFs, and compared to NRMM computed HEMTT performance. HEMTT should be classified in the medium mobility wheeled bundle

The LMTV is classified as a "high mobility wheeled" vehicle, while the MTV is classified as a "medium mobility wheeled" vehicle. Comparison of the cross country performance of these two vehicles using NRMM shows little difference between them and, when compared to the M1025A2 indicates that they should be classified as "high mobility wheeled vehicles" (Figure A22). 


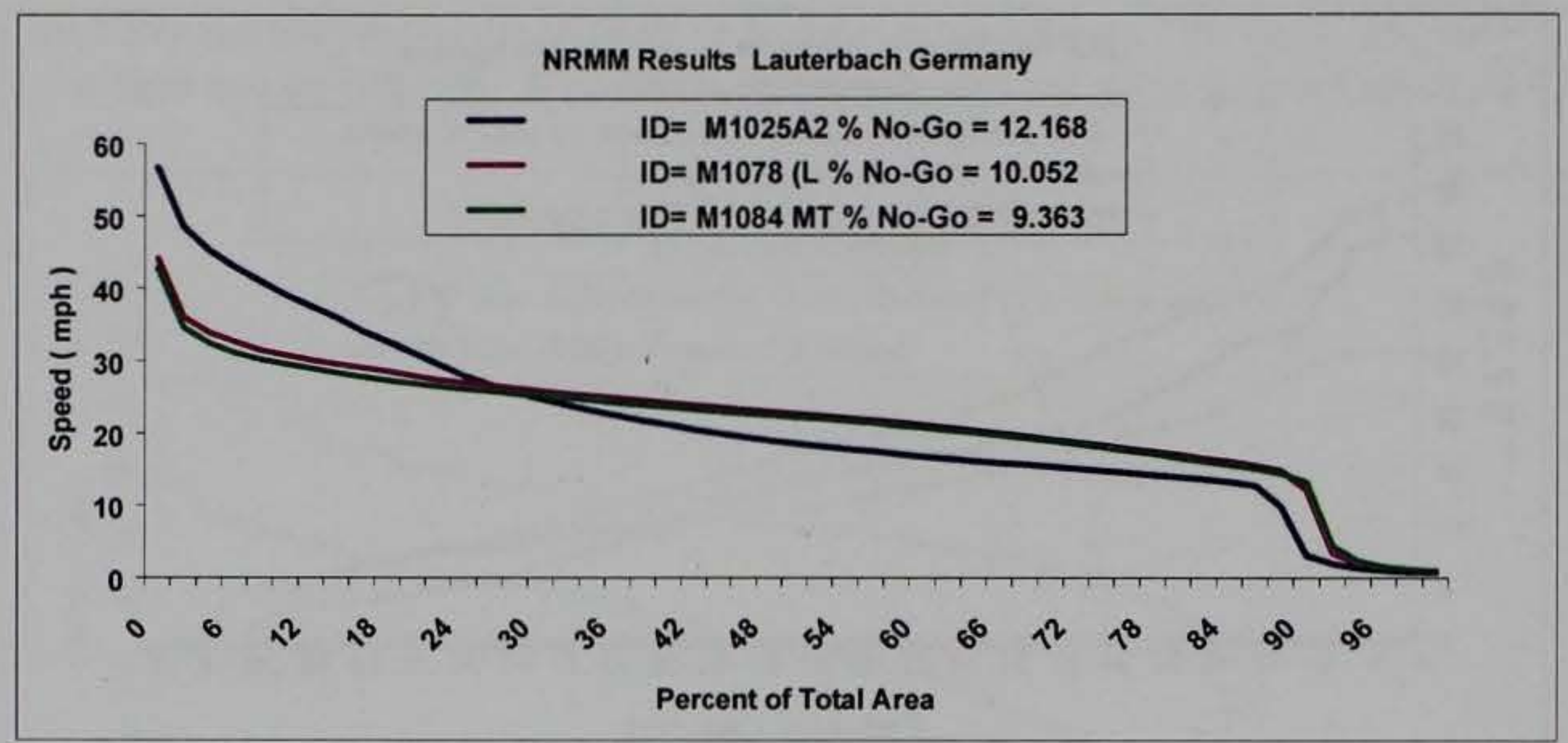

Figure A22. Performance of MTV, LMTV, and M1025A2. The MTV and LMTV should be reclassified as "high mobility wheeled" vehicles

While the "high mobility wheeled" SRF and NRMM simulations of the BRDM-2 are comparable, simulation of other vehicles such as LAV-25, MTV, and LMTV do not compare as well. There are large differences between SRF derived and NRMM computed speeds for these vehicles over a major portion of the terrain (Figures A23 and A24).

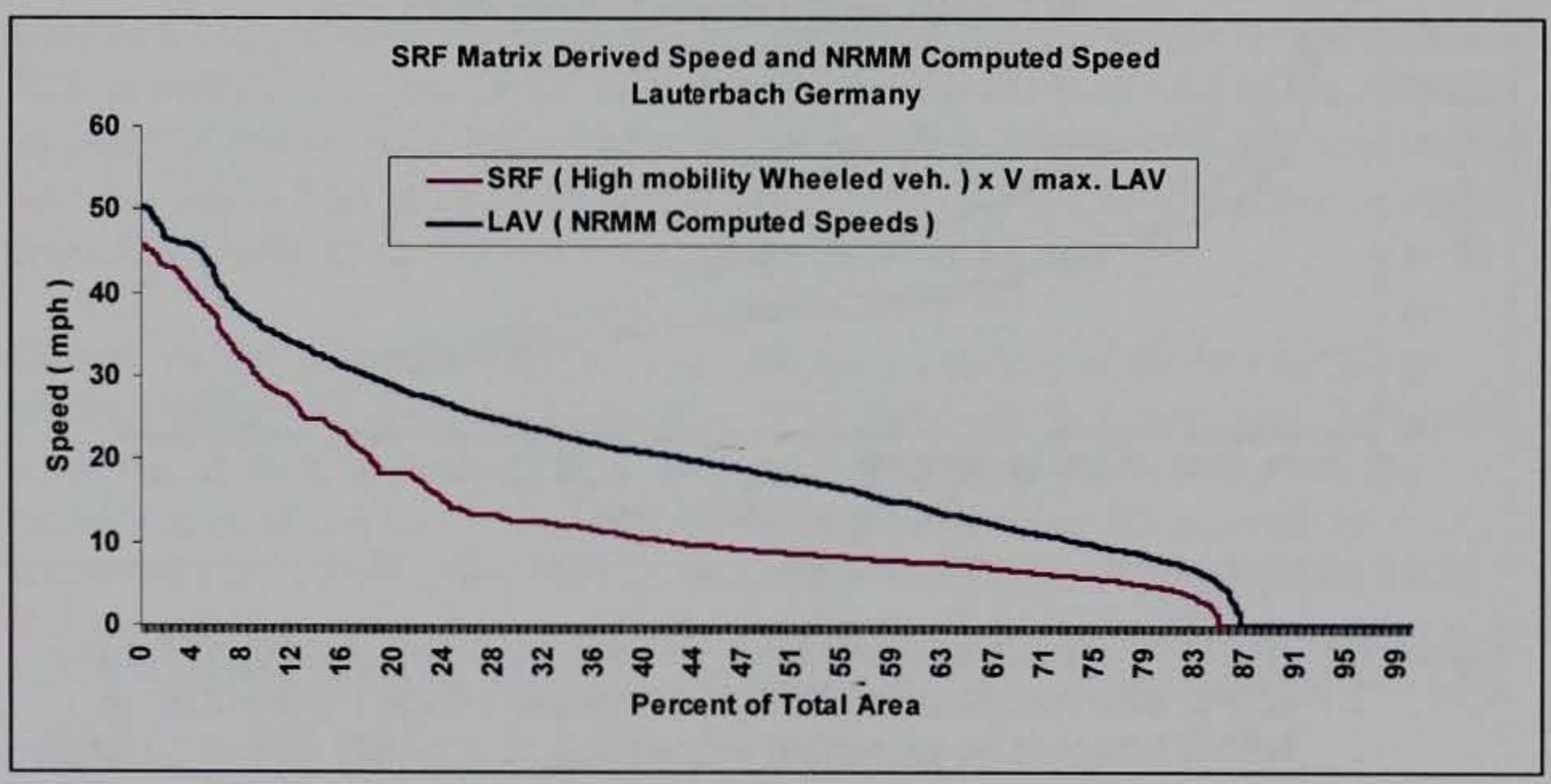

Figure A23. High mobility wheeled SRF, NRMM comparison 


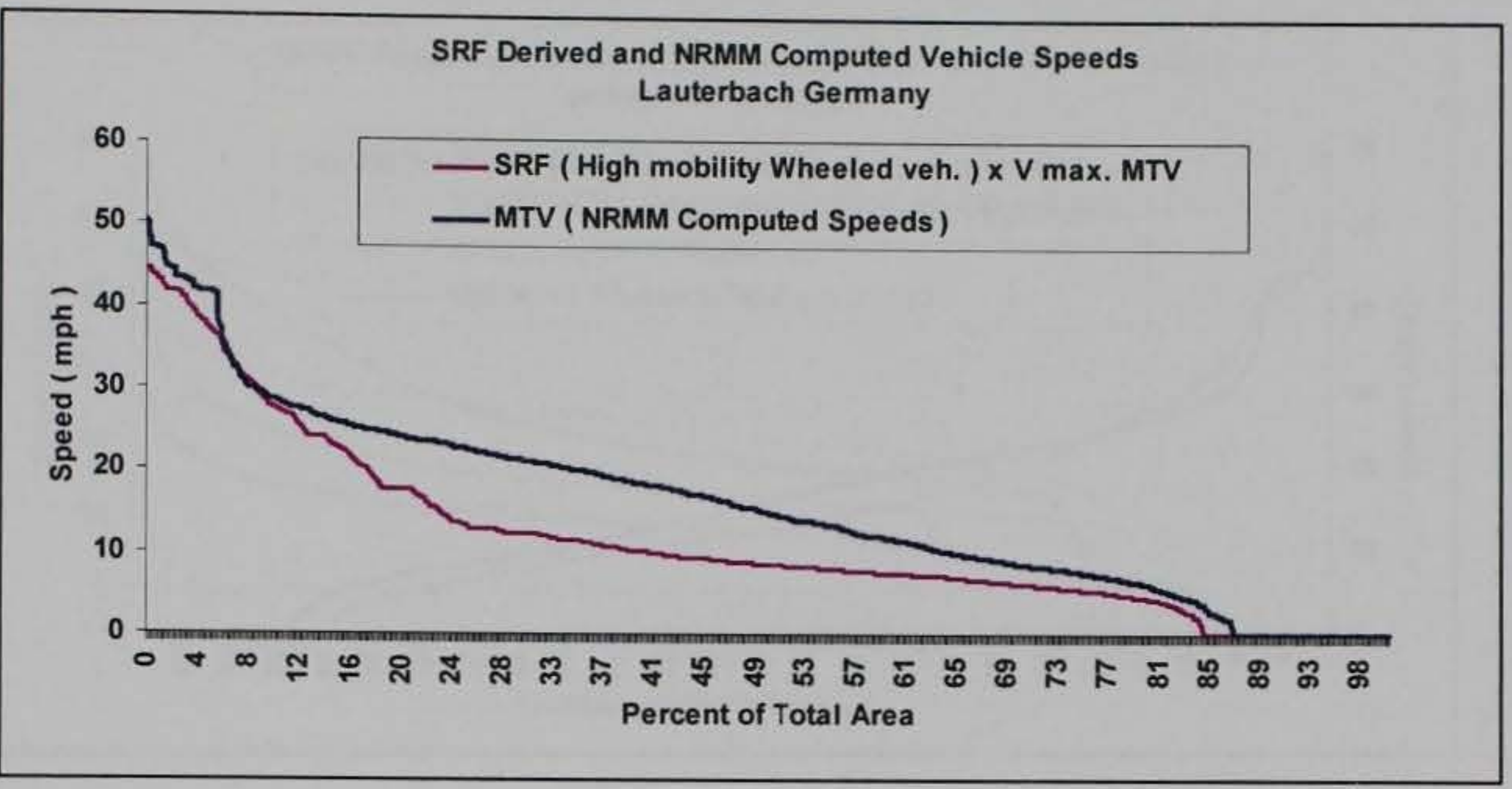

Figure A24. High mobility wheeled SRF, NRMM comparison

The reason for this is that while the M1025A2 is indeed a high mobility wheeled vehicle, it is not the best representative of this bundle. The very high maximum on-road speed ( $82 \mathrm{mph}$ ) of this vehile is the cause of the problem. The SRF matrix is constructed by running NRMM using the representative vehicle (in this case the M1025A2) and then dividing the speed predicted by NRMM on each terrain unit by the maximum on-road speed of the representative vehicle.

Because the maximum on-road speed of the M1025A2 is so high this results in SRFs that are applicable to HMMWVs but are unrealistically low when applied to other vehicles in the bundle.

A speed reduction matrix developed based on the performance of the MTV rather than that of the M1025A2 resulted in SRF derived speeds for the LAV and BRDM-2 which were much closer to the NRMM computed results (Figures A25 and A26).

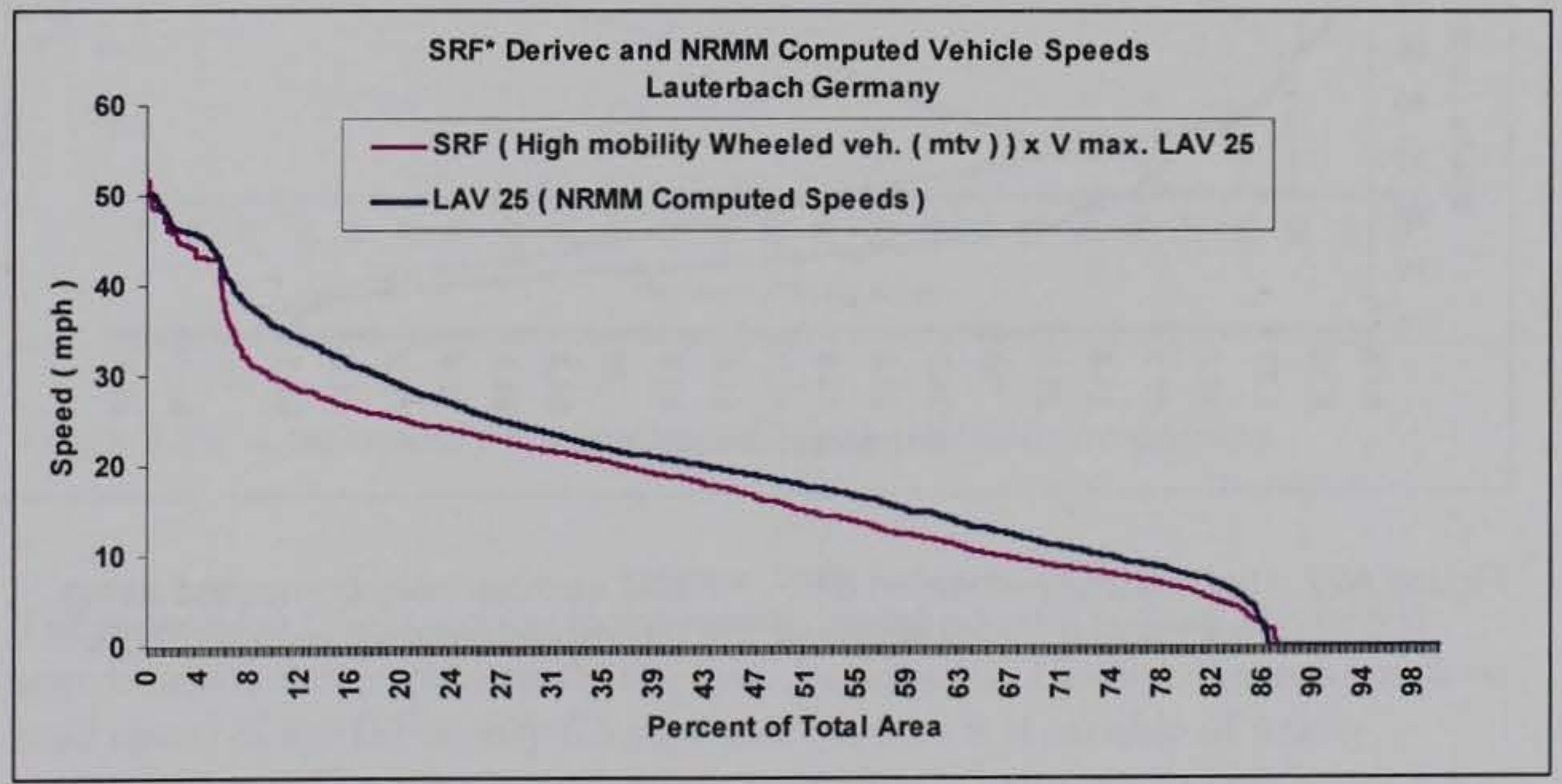

Figure A25. High mobility wheeled SRF, NRMM comparison, computed using new "high mobility wheeled" (MTV) representative vehicle SRFs 


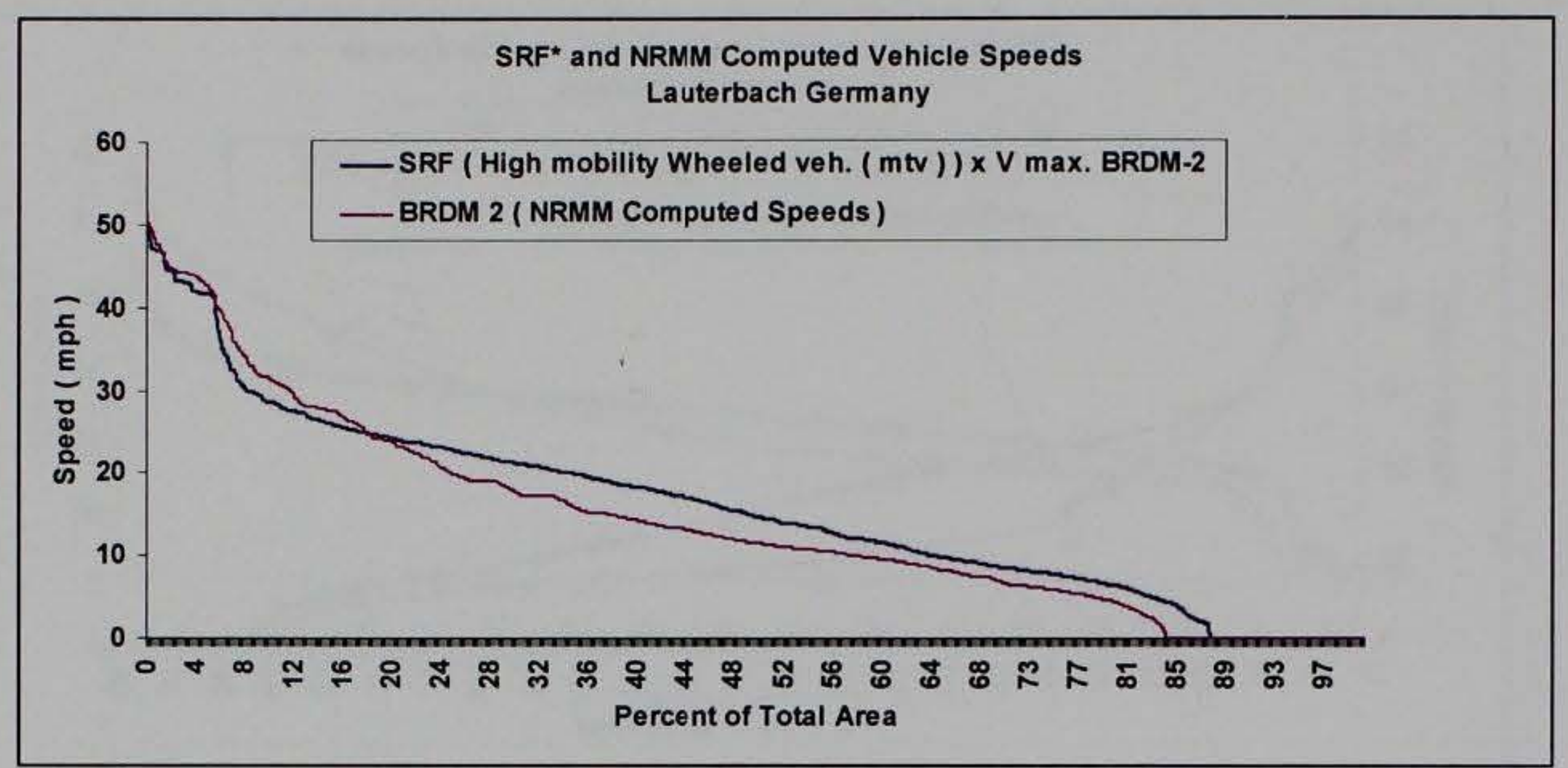

Figure A26. High mobility wheeled SRF, NRMM comparison, computed using new "high mobility wheeled" (MTV) representative vehicle SRFs

However, when the M1025A2 is simulated using the new (MTV) SRFs there are significant differences in SRF and NRMM predicted performance (Figure A27).

NRMM results for the wheeled representative vehicles (Figure A28), and the MTV show that the MTV is a reasonable representative for the "high mobility wheeled" bundle. It is faster than the M1025A2 over about 60 percent of the terrain and encounters fewer no-gos. It is also consistently faster and encounters fewer no-gos than the medium mobility representative vehicle.

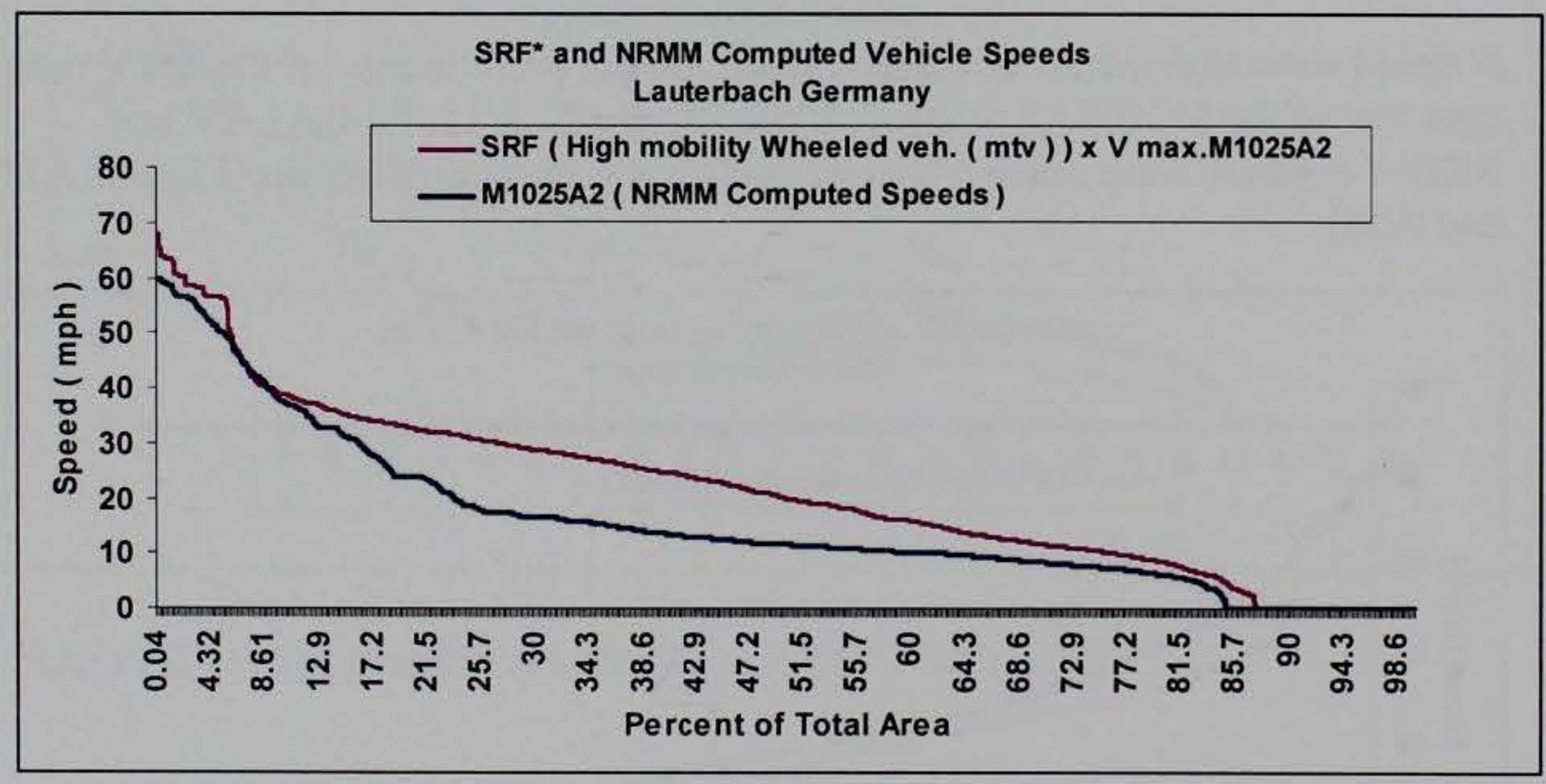

Figure A27. High mobility wheeled SRF, NRMM comparison, computed using new "high mobility wheeled" (MTV) representative vehicle SRFs 


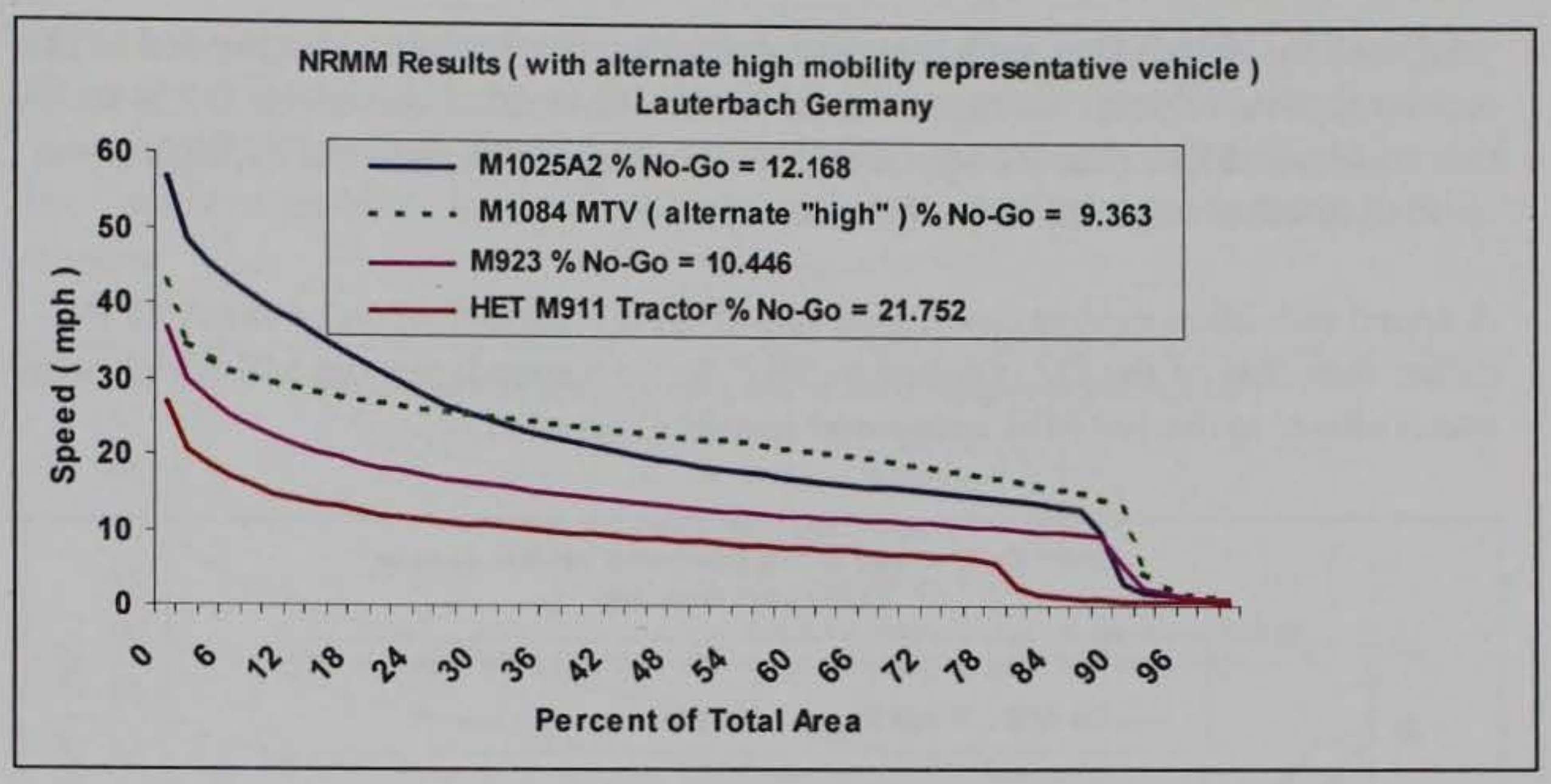

Figure A28. High mobility wheeled vehicles simulated by NRMM with terrain in Lauterbach Germany

The D7 dozer SRF and NRMM computed speeds for the M9 ACE do not compare very well (Figure 19). A second "low mobility tracked" vehicle, the AVLB, was simulated using the D7 SRFs and compared with it's NRMM computed performance (Figure A29). This comparison also shows speeds computed using the SRF method of speed computation which are too high.

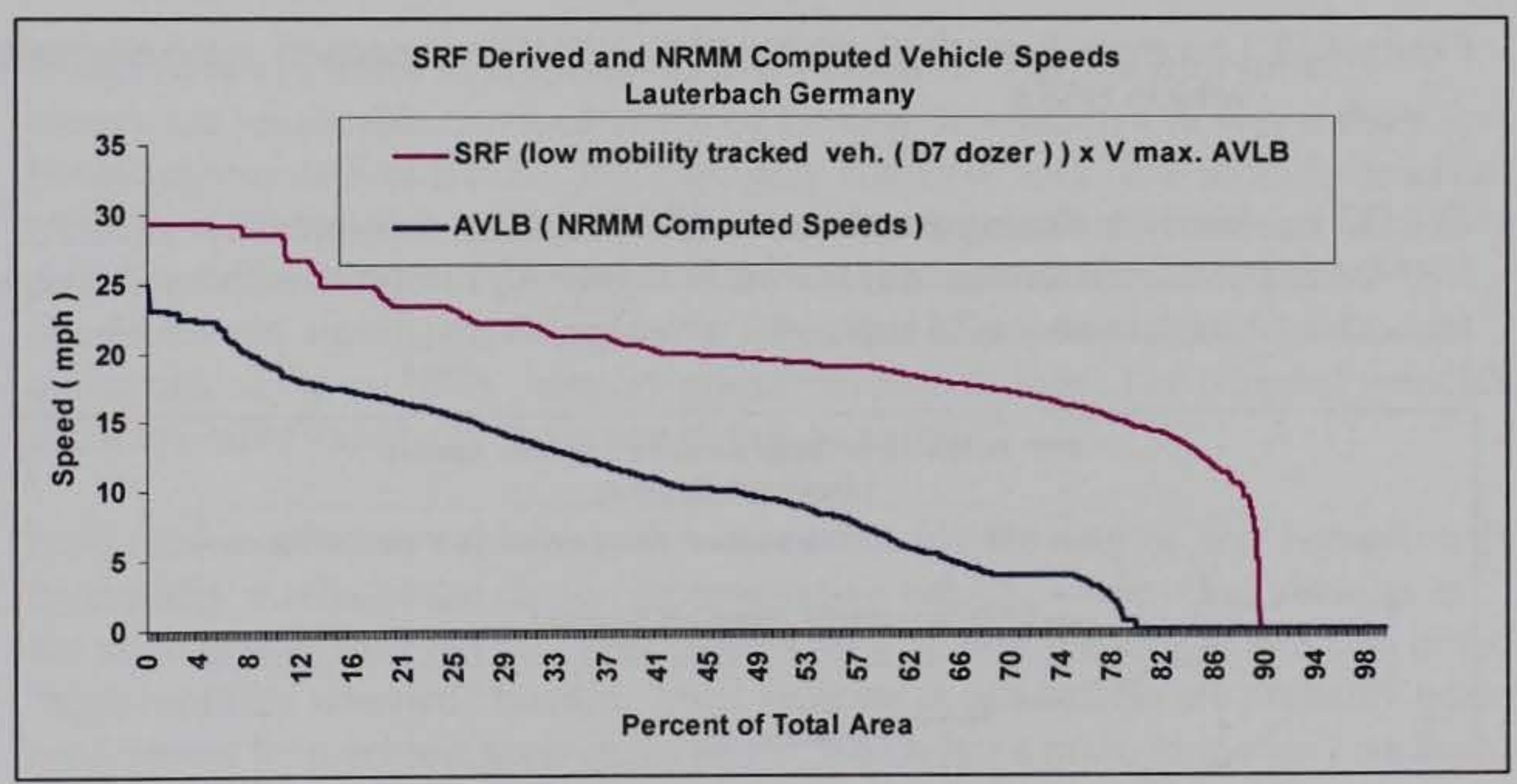

Figure A29. Low mobility tracked vehicle SRF, NRMM comparison

The reason for this is similar to the reason that the M1025A2 SRFs yielded speeds which were consistently too low. In this case, because the maximum on road speed of the D7 is only $6.3 \mathrm{mph}$ and because it is capable of nearly maximum speed on all off road terrain untill it reaches a no-go condition, it's SRFs are all very high. The SRF matrix is constructed by running NRMM using the representative vehicle (in this case the D7) and then dividing the speed 
predicted by NRMM on each terrain unit by the maximum on-road speed of the representative vehicle. Because the maximum on-road speed of the D7 is so low this results in SRFs that are applicable to D7s but are unrealistically high when applied to other vehicles in the bundle.

A speed reduction matrix developed based on the performance of the AVLB rather than that of the D7 resulted in SRF derived speeds for the M9 which were much closer to the NRMM computed results (Figure A30).

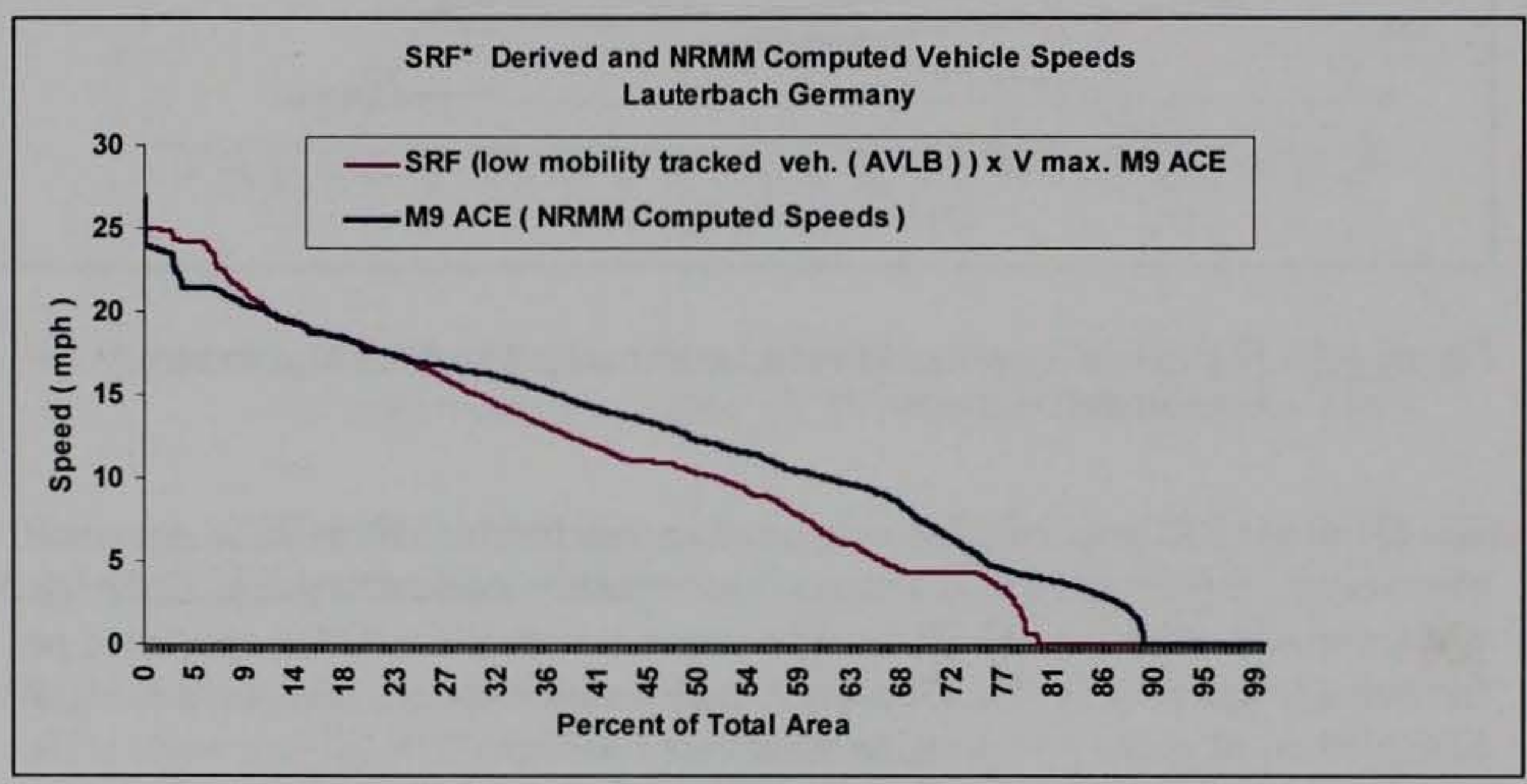

Figure A30. Low mobility tracked vehicle SRF, NRMM comparison using alternate vehicle SRFs

The D7 was simulated using the alternate AVLB SRFs. A comparison of SRF, NRMM computed performance is shown in Figure A31. Speed differences range from about $1 \mathrm{mph}$ to about $2.5 \mathrm{mph}$.

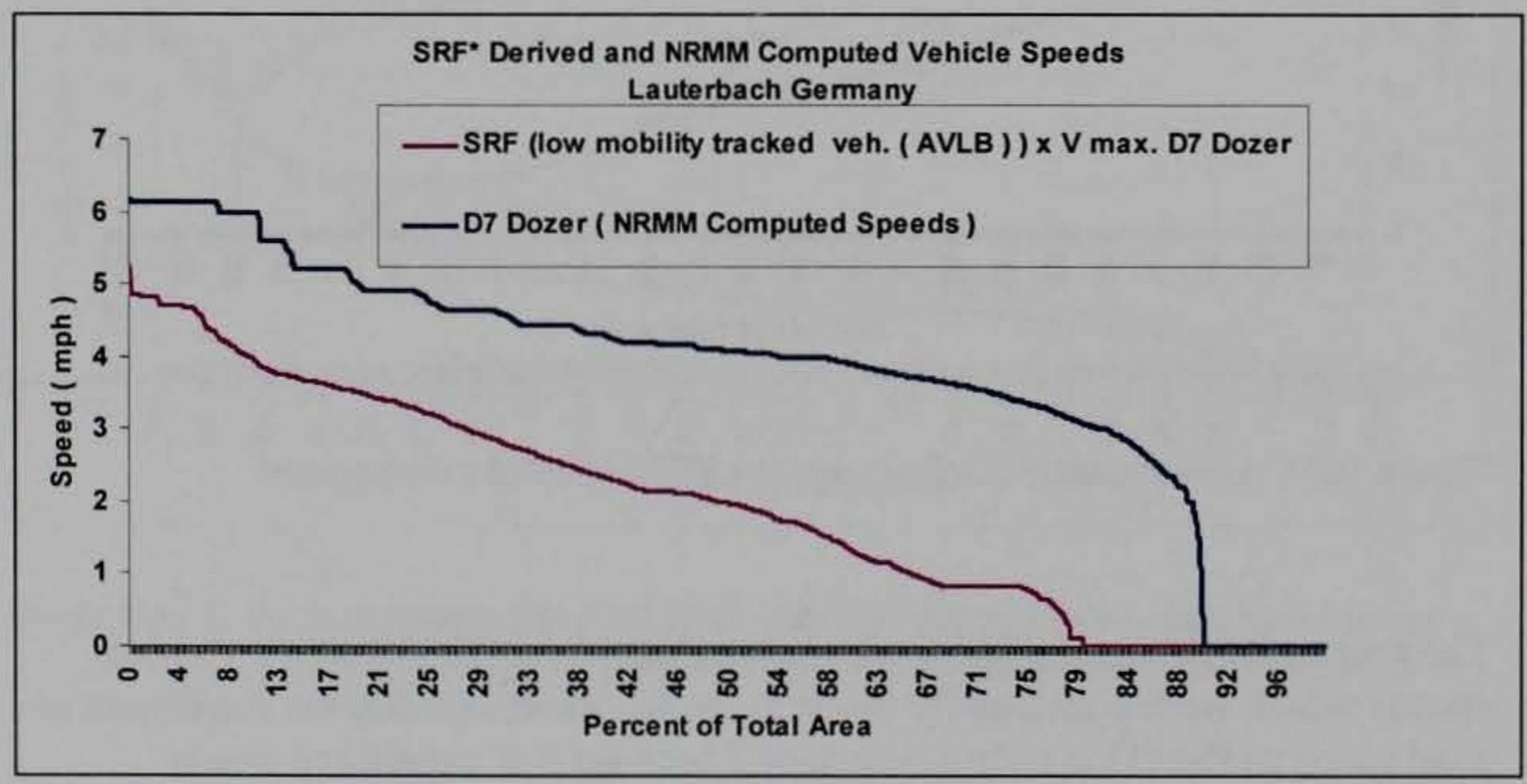

Figure A31. Low mobility tracked vehicle SRF, NRMM comparison using alternate vehicle SRFs 
A plot of NRMM results for the tracked representative vehicles in Lauterbach Germany (Figure A32) shows that the AVLB is a good representative for the "low mobility tracked" bundle. It's speeds are significantly lower than those of the "medium mobility tracked" vehicle, and it encounters significantly more no-gos.

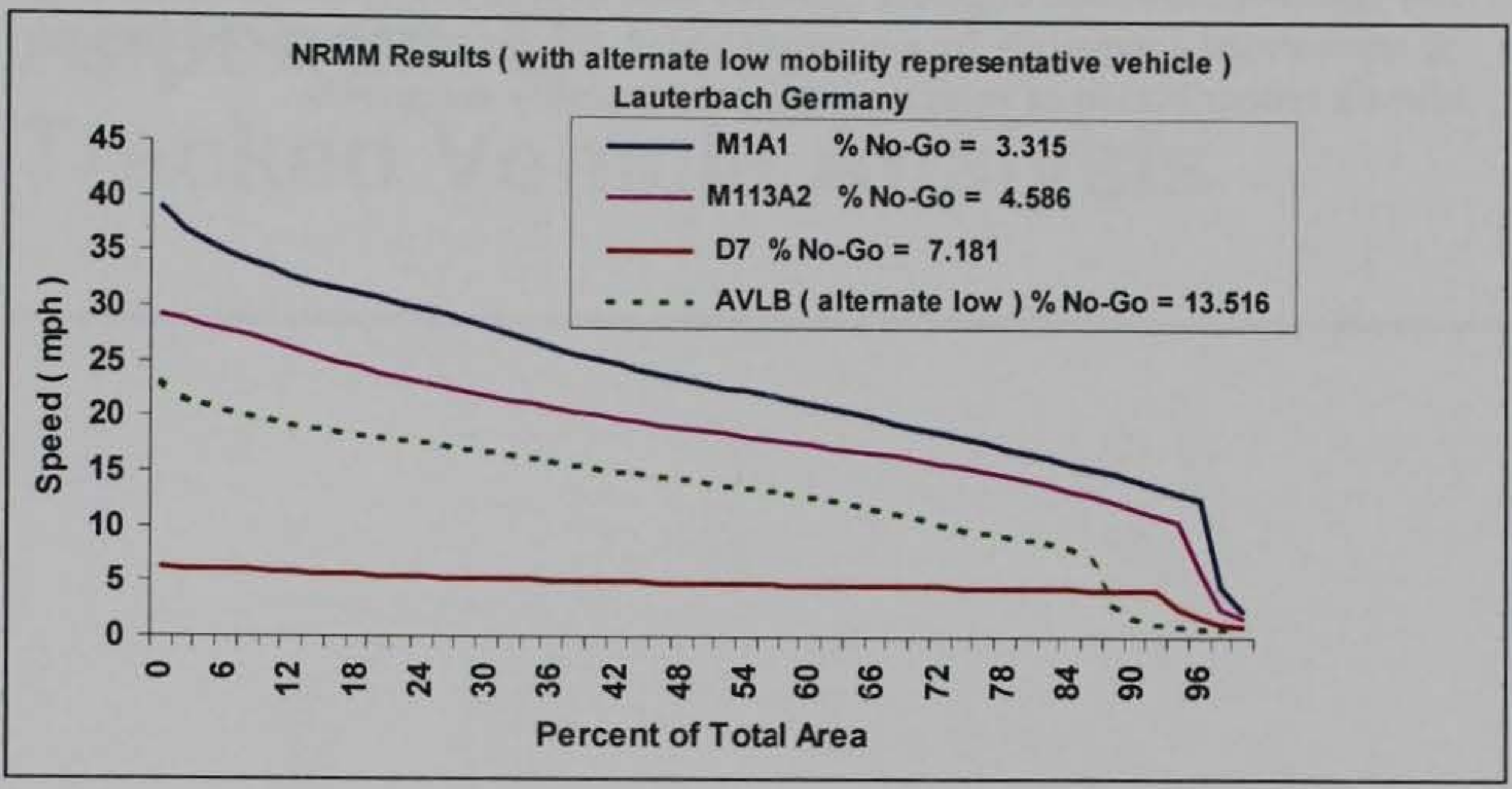

Figure A32. NRMM results for tracked representative vehicles in Lauterbach Germany

Conclusions (Choice of Representative Vehicles). The vehicles initially chosen are reasonable representatives of the mobility bundles, however there are better representatives for the "high mobility wheeled" and "low mobility tracked" bundles. NRMM simulated performance of the representative vehicles on widely differing types of terrain (temperate European, desert, mountainous) show consistent and significant decreases in speed and in some cases increases in nogo terrain, as the mobility category goes from high to low. The wheeled vehicles show the most variability from one terrain to another.

Performance of some vehicles within a mobility bundle may be well represented by the SRF matrix of the chosen representative vehicle, while other vehicles in the same bundle may not be. This is illustrated by performance of vehicles in the "high mobility wheeled" bundle. Most vehicles in this bundle are probably better represented by a vehicle such as the MTV, which has a more moderate maximum on-road speed than the current representative (M1025A2).

The D7 is not the best representative of the low mobility tracked bundle. It's cross country speed profile is unusual in that it is capable of nearly maximum speed until it begins to encounter no-go conditions. It's maximum speed is also unusually low at $6.3 \mathrm{mph}$. A more suitable representative vehicle for this bundle is the AVLB (see Figure A32).

Conclusions (SRF Method of Speed Prediction). The SRF speed computation method can result in speeds very close to those predicted for a vehicle by NRMM. However, if a vehicle is not classified in the correct mobility bundle 
then speed prediction will not be as good. Fidelity of the SRF simulation method is highly dependent on vehicles being classified in the appropriate mobility bundles. The mobility bundle document should be reviewed and vehicles reclassified as needed. There are very large numbers of vehicles classified as high and medium mobility and extremely few classified as low mobility. Very low speed construction/engineer vehicles such as fork lifts and bulldozers could be represented adequately by a constant speed, this would free this bundle and allow a redistribution of vehicles within the mobility categories. 


\section{Appendix B Tracked Vehicle Analysis}


Response: tact high (kph)

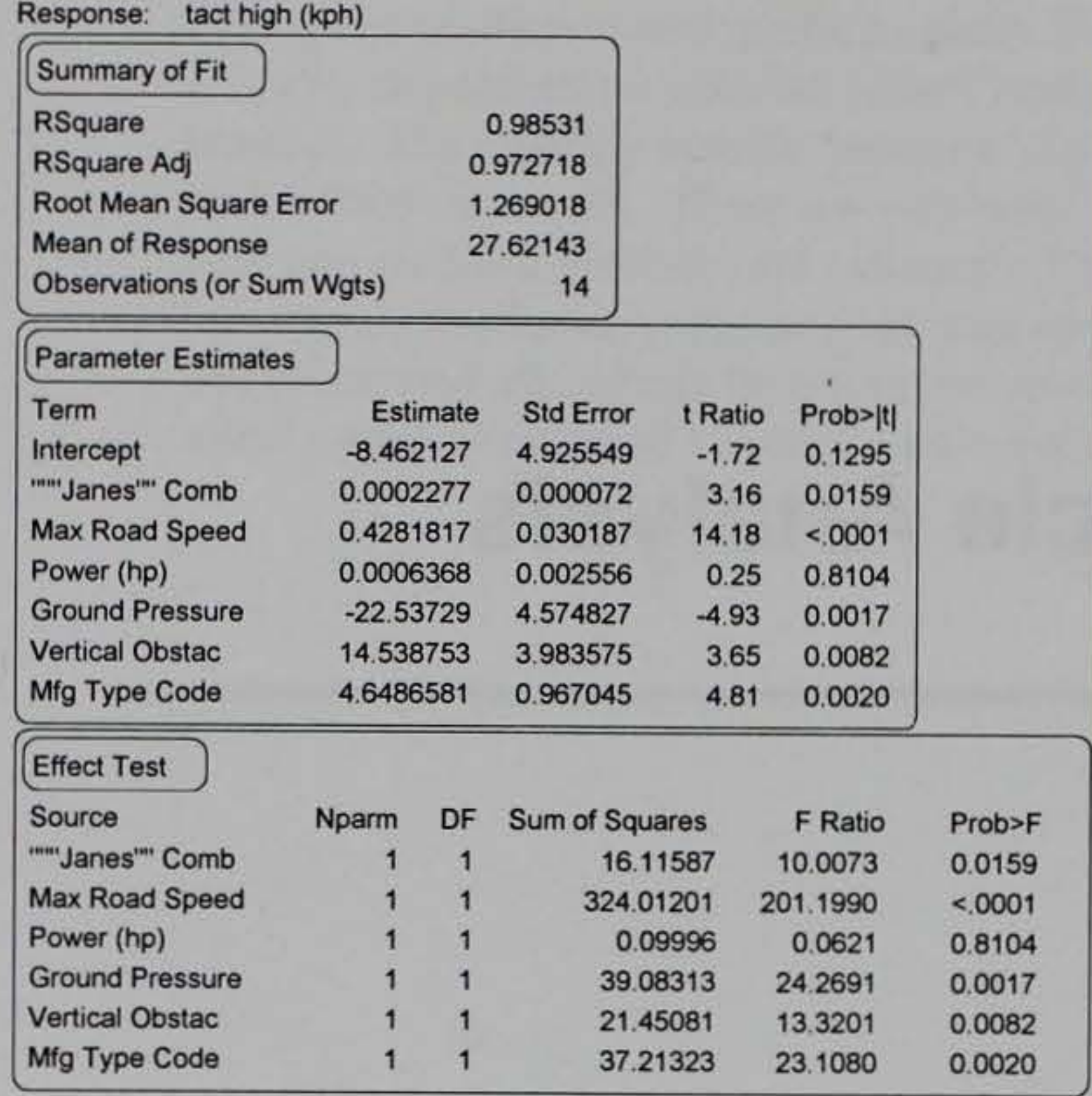

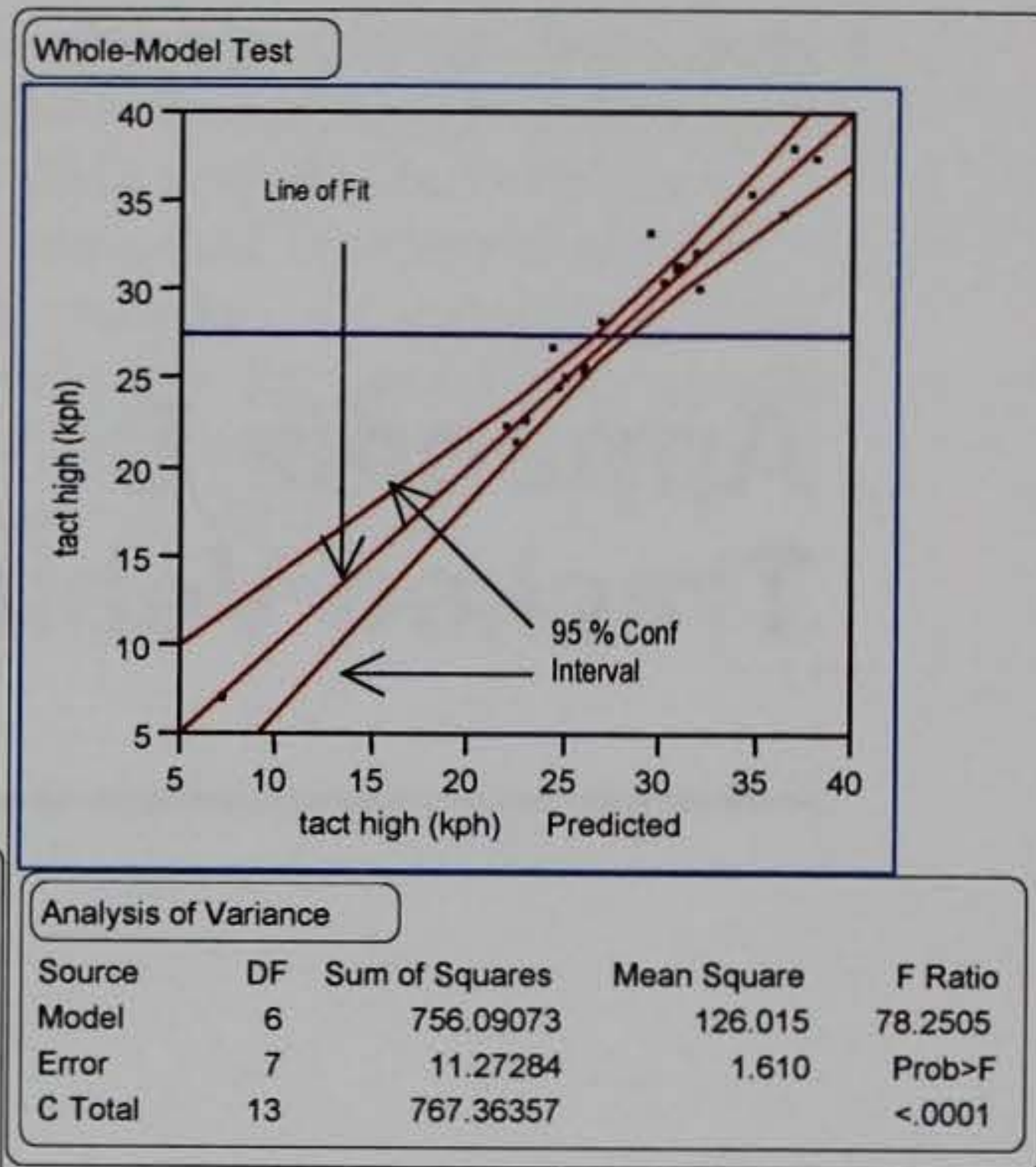

Figure B1. Results of regression analysis for tracked vehicles (Iteration 1)

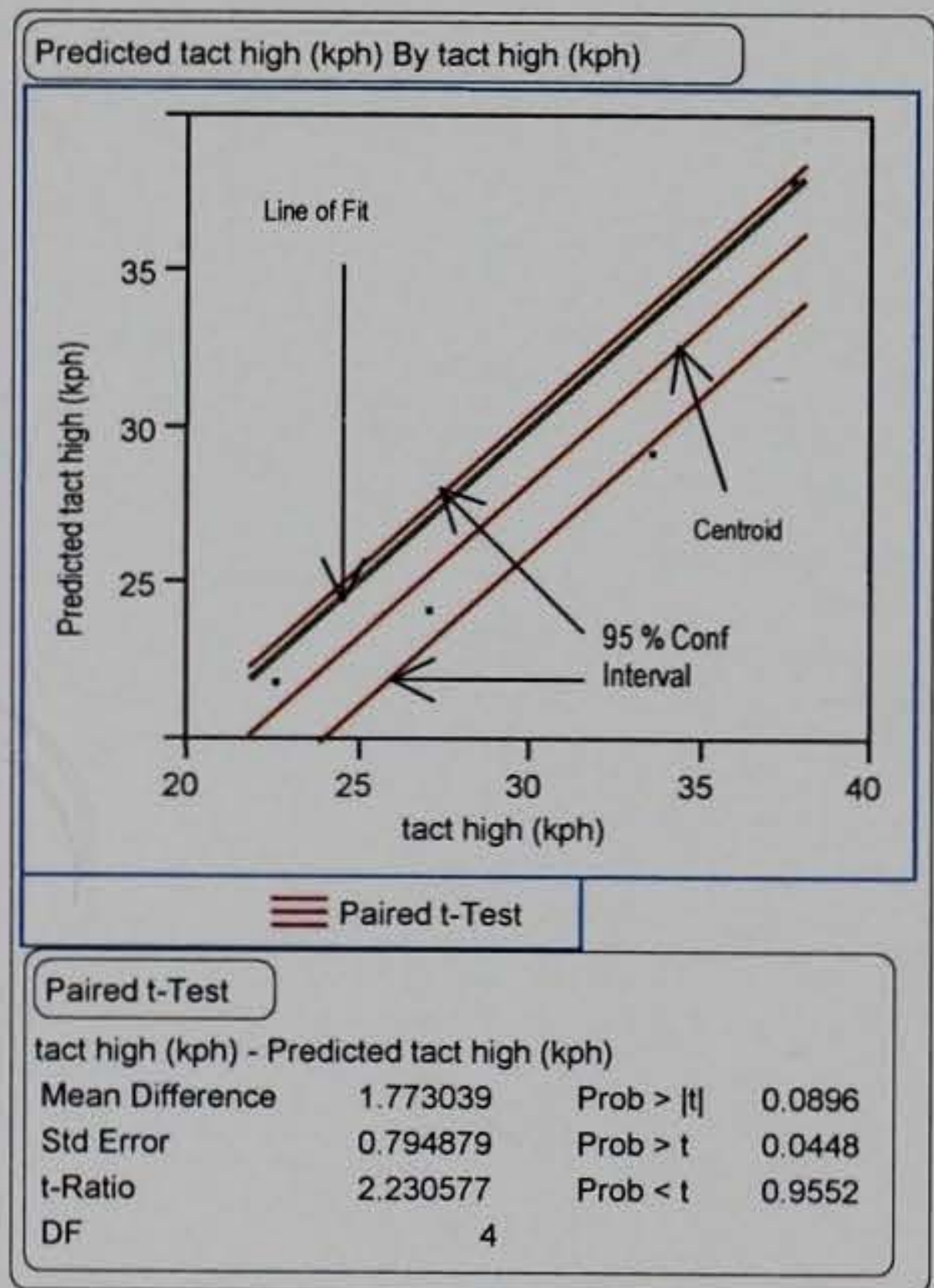

Figure B2. Results of paired t test for tracked vehicles (Iteration 1) 


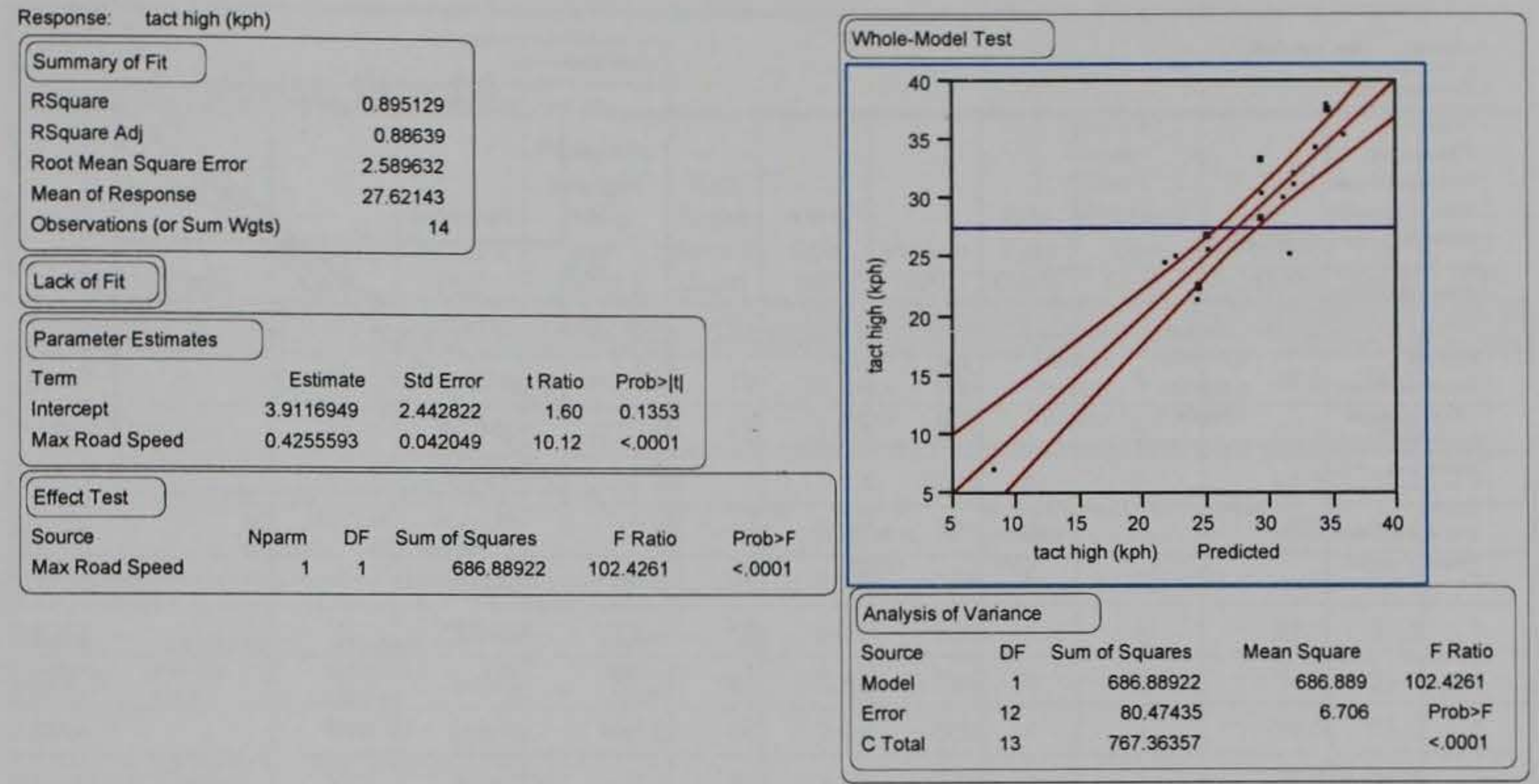

Figure B3. Results of regression analysis for tracked vehicles (Iteration 2)

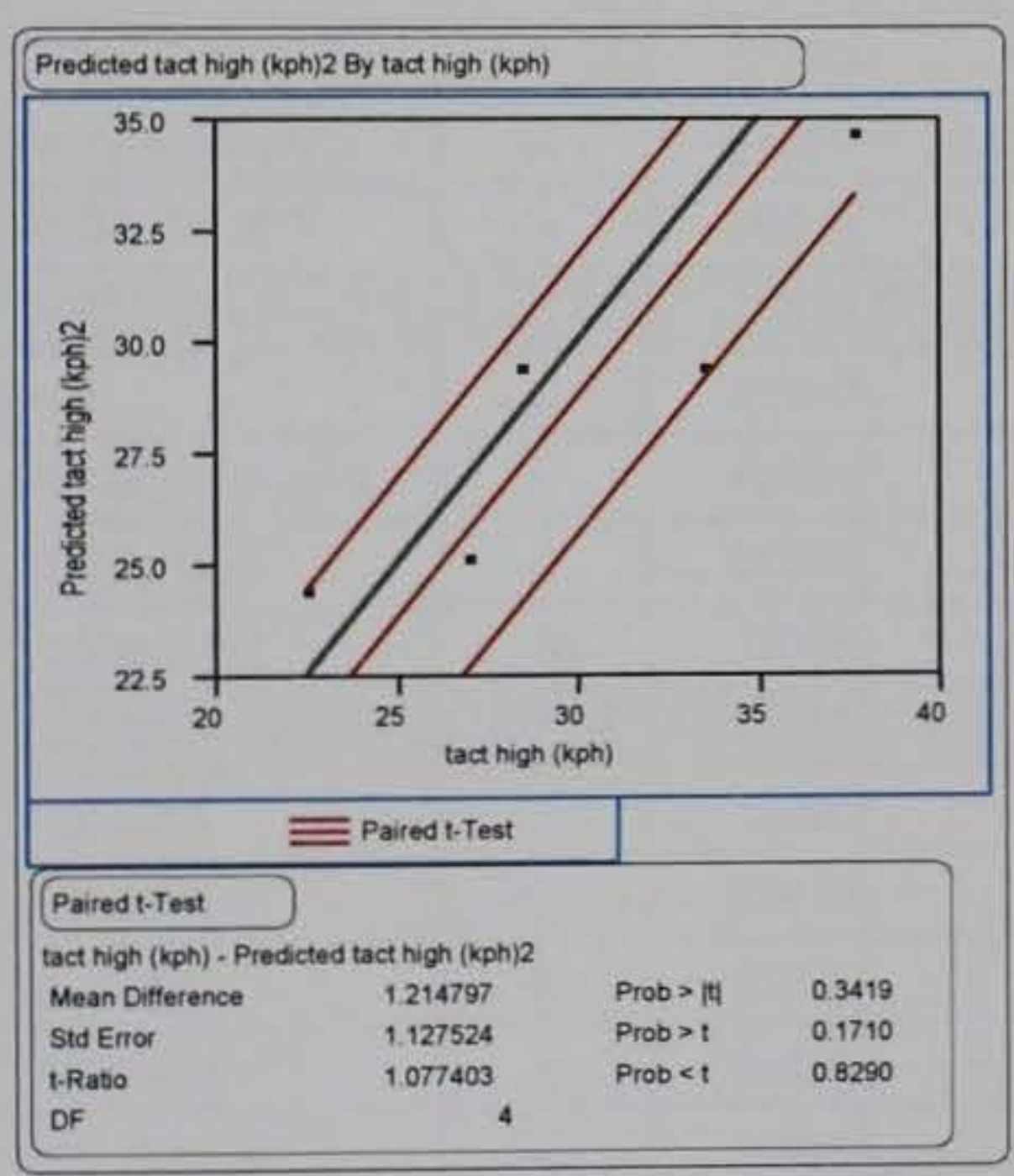

Figure B4. Results of paired $t$ test for tracked vehicles (Iteration 2) 
Response: tact high (kph)

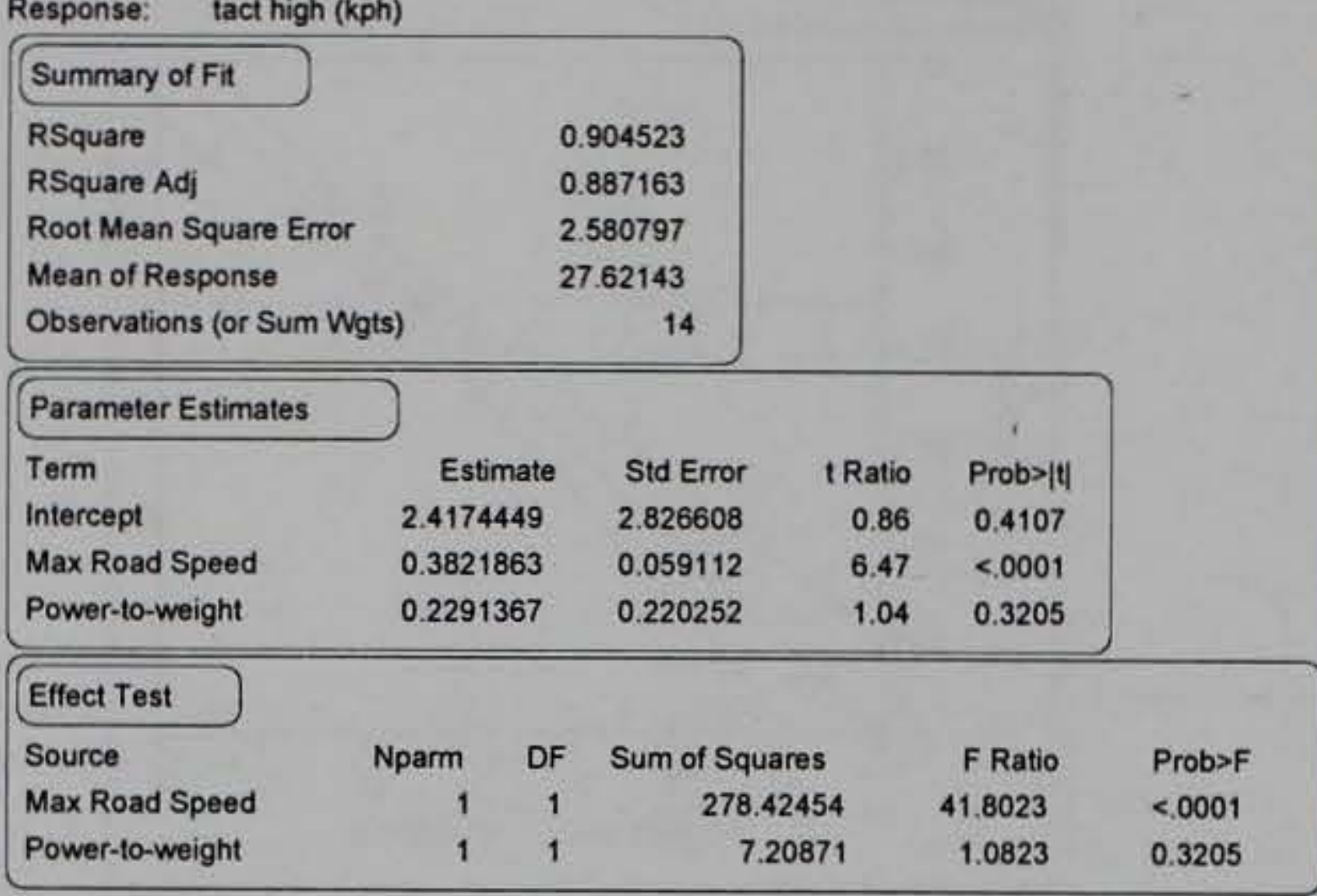

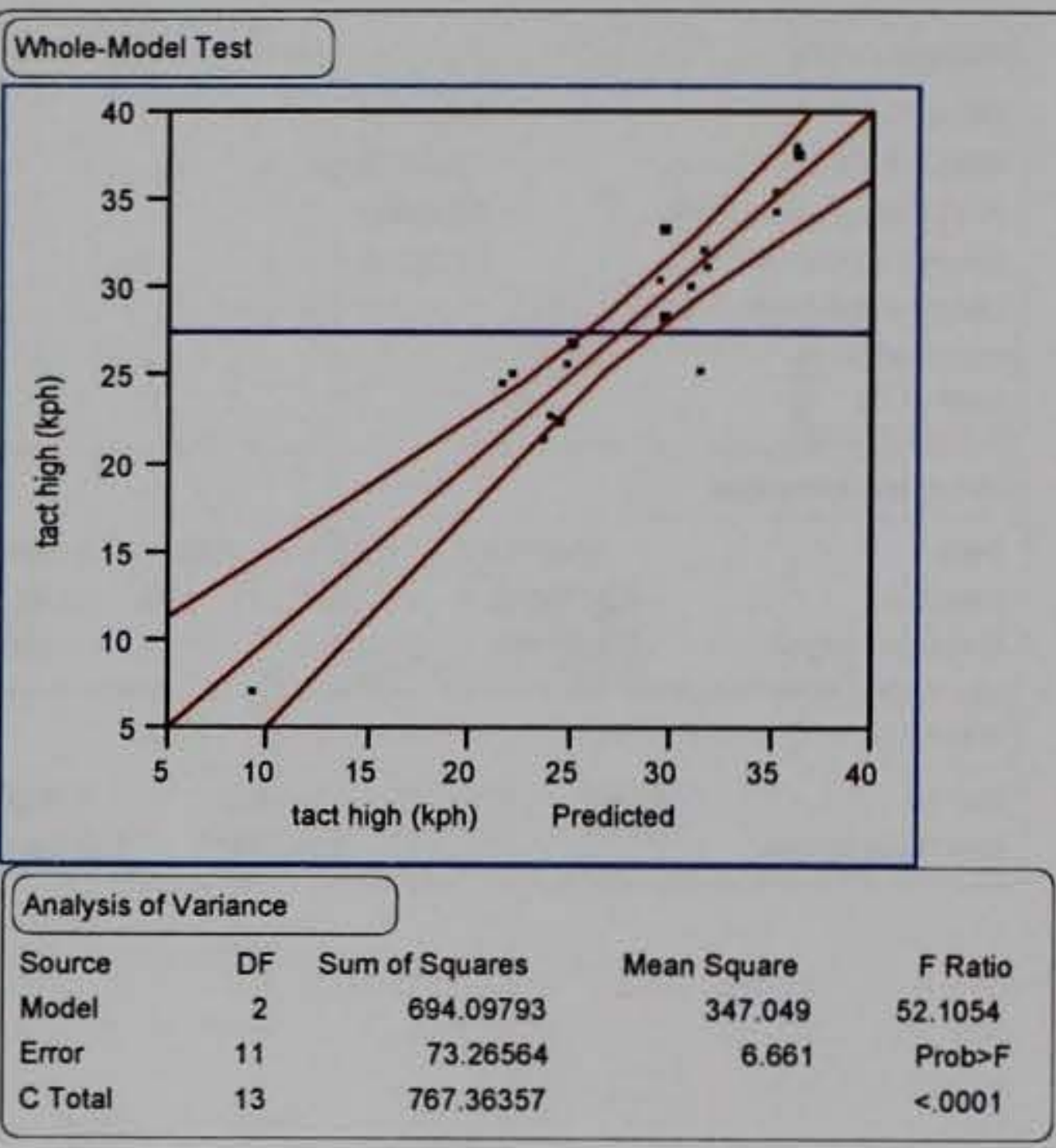

Figure B5. Results of regression analysis for tracked vehicles (Iteration 3)

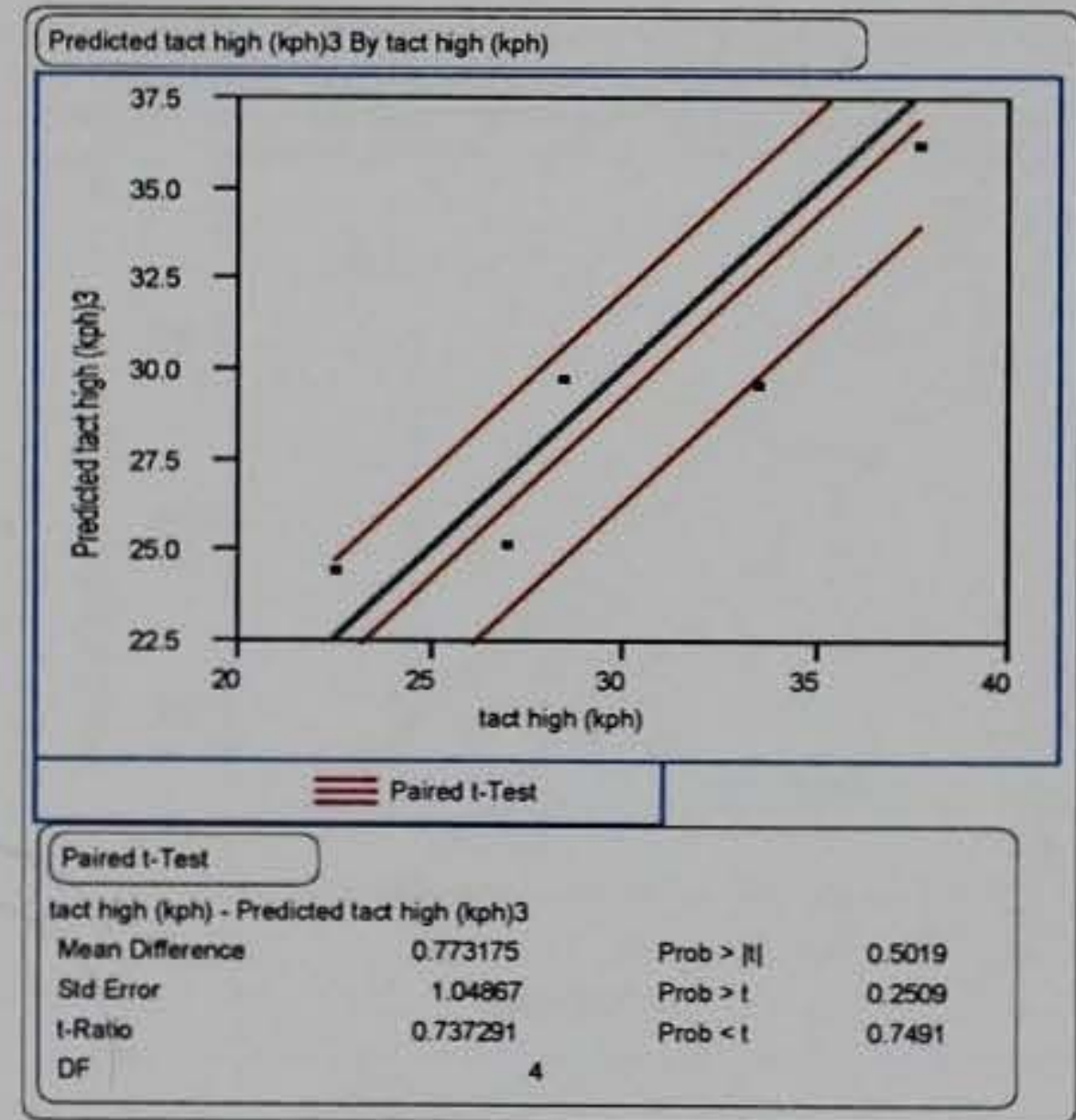

Figure B6. Results of paired $t$ test for tracked vehicles (Iteration 3 ) 
Table B1

Tracked Vehicles, Bins 1-3

\begin{tabular}{|c|c|c|c|c|c|c|c|c|c|c|c|}
\hline Tracked Vehicle & $\begin{array}{c}\text { NRMM II } \\
\text { File }\end{array}$ & $\begin{array}{c}\text { Combat } \\
\text { Weight } \\
(\mathrm{kg})\end{array}$ & $\begin{array}{l}\text { Power to } \\
\text { Weight } \\
\text { Ratio } \\
\text { (hp/ } \\
\text { ton) } \\
\end{array}$ & \begin{tabular}{|c|} 
Max \\
Road \\
Speed \\
$(\mathrm{kph})$ \\
\end{tabular} & $\begin{array}{l}\text { Vert } \\
\text { Obs } \\
\text { (m) } \\
\end{array}$ & $\begin{array}{c}\text { Power } \\
\text { (hp) }\end{array}$ & $\begin{array}{l}\text { Mfg } \\
\text { Type } \\
\text { Code } \\
\end{array}$ & $\begin{array}{c}\text { Primary } \\
\text { Use } \\
\text { Code } \\
\end{array}$ & $\begin{array}{c}\text { NRMMII } \\
\mathrm{Y}_{\text {TH }}(\mathrm{kph}) \\
\end{array}$ & $\begin{array}{c}\text { Pred. } \\
Y_{\mathrm{TH}} \\
(\mathrm{kph}) \\
\end{array}$ & BIN \\
\hline Leopard II & $Y$ & 55150 & 27.0 & 72 & 1.10 & 1641 & 1 & 5 & 37.7 & 36.3 & 1 \\
\hline $\mathrm{M} 1 \mathrm{~A} 1^{1,2,3}$ & $Y$ & 54545 & 27.0 & 72 & 1.24 & 1500 & 1 & 5 & 38.2 & 36.1 & 1 \\
\hline AMX 40 LeClerc & $\mathrm{N}$ & 43700 & 30.0 & 70 & 1.00 & 1445 & 1 & 5 & & 36.0 & 1 \\
\hline T80 & $Y$ & 42500 & 25.9 & 70 & 1.00 & 1213 & 2 & 5 & 34.5 & 35.1 & 1 \\
\hline T64 & $Y$ & 39500 & 17.7 & 75 & 0.80 & 771 & 2 & 5 & 35.6 & 35.1 & 1 \\
\hline Leopard I & $\mathrm{N}$ & 40000 & 20.8 & 65 & 1.15 & 915 & 1 & 5 & & 32.0 & 1 \\
\hline $\mathrm{M} 2 \mathrm{~A} 2$ & $\mathrm{Y}$ & 30000 & 18.1 & 66 & 0.91 & 600 & 1 & 4 & 31.5 & 31.8 & 1 \\
\hline $\mathrm{AM} \times 30$ & $\mathrm{~N}$ & 36000 & 20.0 & 65 & 0.93 & 794 & 1 & 5 & & 31.8 & 1 \\
\hline$M 2 A 1^{1,3}$ & $Y$ & 25940 & 17.5 & 66 & 0.91 & 500 & 1 & 4 & 32.3 & 31.6 & 1 \\
\hline MarderA3 /Roland & $Y$ & 35000 & 18.0 & 65 & 1.00 & 600 & 1 & 4 & 25.6 & 31.4 & 1 \\
\hline M270 MLRS & $Y$ & 25191 & 18.0 & 64 & 1.00 & 500 & 1 & 4 & 30.3 & 31.0 & 2 \\
\hline Challenger & $Y$ & 62000 & 19.4 & 60 & 0.85 & 1322 & 1 & 5 & 28.5 & 29.8 & 2 \\
\hline $\mathrm{T} 72$ & $Y$ & 44500 & 18.9 & 60 & 0.85 & 927 & 2 & 5 & 33.5 & 29.6 & 2 \\
\hline GMZ Mine Layer & $\mathrm{N}$ & 28500 & 18.4 & 60 & 0.70 & 579 & 2 & 4 & & 29.5 & 2 \\
\hline $2 \mathrm{~S} 3152 \mathrm{~mm}$ SPH & $Y$ & 27500 & 17.3 & 60 & 0.70 & 525 & 2 & 4 & 30.7 & 29.3 & 2 \\
\hline Merkava Mk 3 & $\mathrm{~N}$ & 61000 & 19.7 & 55 & 1.00 & 1323 & 1 & 5 & & 27.9 & 2 \\
\hline M109A1B SPH & $\mathrm{N}$ & 24948 & 14.7 & 56 & 0.53 & 405 & 1 & 4 & & 27.3 & 2 \\
\hline T55 & $Y$ & 36000 & 16.1 & 50 & 0.80 & 639 & 2 & 5 & 27.0 & 25.2 & 3 \\
\hline T69 & $\mathrm{N}$ & 37000 & 15.9 & 50 & 0.80 & 648 & 2 & 5 & & 25.1 & 3 \\
\hline T62 w/o Rct-Arm & $Y$ & 40000 & 14.5 & 50 & 0.80 & 639 & 2 & 5 & 25.0 & 24.8 & 3 \\
\hline T54/Type 59 & $\mathrm{~N}$ & 36000 & 14.4 & 50 & 0.79 & 573 & 2 & 5 & & 24.8 & 3 \\
\hline T55/MTU-20 & $\mathrm{N}$ & 37000 & 14.2 & 50 & 0.80 & 580 & 2 & 4 & & 24.8 & 3 \\
\hline M48A5 & $Y$ & 48987 & 15.9 & 48 & 0.92 & 750 & 1 & 5 & 22.6 & 24.5 & 3 \\
\hline T55/IMR & $Y$ & 34000 & 15.5 & 48 & 0.80 & 580 & 2 & 5 & 25.9 & 24.3 & 3 \\
\hline M60A3 & $Y$ & 52617 & 14.2 & 48 & 0.91 & 750 & 1 & 5 & 22.9 & 24.1 & 3 \\
\hline Chieftain & $\mathrm{N}$ & 55000 & 13.6 & 48 & 0.91 & 826 & 1 & 5 & & 23.9 & 3 \\
\hline M60/AVLB $^{1,2,3}$ & $Y$ & 55205 & 12.3 & 48 & 0.91 & 750 & 1 & 4 & 21.6 & 23.6 & 3 \\
\hline ZSU-23-4/SA-6 & $Y$ & 20500 & 12.4 & 44 & 1.10 & 280 & 2 & 4 & 25.5 & 22.1 & 3 \\
\hline M88A1 & $Y$ & 50848 & 13.4 & 42 & 1.07 & 750 & 1 & 4 & 24.9 & 21.8 & 3 \\
\hline D7-G & $Y$ & 14456 & 12.6 & 10 & 1.00 & 200 & 1 & 4 & 7.3 & 9.1 & 3 \\
\hline \multicolumn{12}{|c|}{$\begin{array}{l}\text { Note: Grayed rows indicate a vehicle not part of the regression formulation, } \\
\text { prediction and the regression prediction. } \\
\text { Representative vehicle for WARSIM \& JWARS by ERDC, October } 2000 . \\
2 \text { Representative vehicle for WARSIM selected by AMSAA, October } 1998 . \\
\text { Bepresentative vehicle for CCTT-SAF. }\end{array}$} \\
\hline
\end{tabular}




\begin{tabular}{|c|c|c|c|c|c|c|c|}
\hline \multicolumn{8}{|c|}{$\begin{array}{l}\text { Table B2 } \\
\text { Tracked Amphibious Combat Vehicle, Bin } 10\end{array}$} \\
\hline Tracked Vehicle & $\begin{array}{l}\text { NRMMII } \\
\text { File Exist }\end{array}$ & $\begin{array}{c}\text { Combat Weight } \\
(\mathrm{kg})\end{array}$ & $\begin{array}{c}\text { Max Road } \\
\text { Speed (kph) }\end{array}$ & \begin{tabular}{|c|} 
Max Grad \\
$\%$ \\
\end{tabular} & $\begin{array}{l}\text { Power } \\
\text { (hp) }\end{array}$ & $\begin{array}{l}\text { Mfg Type } \\
\text { Code }\end{array}$ & $\begin{array}{c}\text { Primary Use } \\
\text { Code }\end{array}$ \\
\hline $\mathrm{M} 113 \mathrm{~A} 2^{1,2,3}$ & $\mathrm{Y}$ & \begin{tabular}{|r|}
11253 \\
\end{tabular} & 61 & 60 & 212 & 1 & 2 \\
\hline M551 & $Y$ & 15830 & 70 & 60 & 300 & 1 & 2 \\
\hline 259 & $\mathrm{Y}$ & 9000 & 60 & 60 & 300 & 2 & 2 \\
\hline M9ACE & $\mathrm{Y}$ & 24500 & 48 & 60 & 295 & 1 & 2 \\
\hline BMP-2 & Y & 14300 & 65 & 60 & 320 & 2 & 2 \\
\hline BMP-1MZ501 & $Y$ & 13500 & 80 & 60 & 300 & 2 & 2 \\
\hline $2 \mathrm{~S} 1122 \mathrm{~mm}$ SPH & $Y$ & 16000 & 60 & 60 & 240 & 2 & 2 \\
\hline PT76 & $Y$ & 14600 & 44 & 70 & 240 & 2 & 2 \\
\hline BMP-3 & $\mathrm{N}$ & 18700 & 70 & 60 & 551 & 2 & 2 \\
\hline YW531H (PRC) & $\mathrm{N}$ & 13600 & 65 & 60 & 352 & 2 & 2 \\
\hline BMD-3 & $\mathrm{N}$ & 13200 & 70 & 60 & 495 & 2 & 2 \\
\hline BMD-1 & $\mathrm{N}$ & 7500 & 70 & 60 & 265 & 2 & 2 \\
\hline BTR-50 & $\mathrm{N}$ & 14200 & 44 & 70 & 265 & 2 & 2 \\
\hline AAVP7A1 & $\mathrm{N}$ & 22838 & 64 & 60 & 441 & 1 & 2 \\
\hline BVP M80A & $\mathrm{N}$ & 14000 & 64 & 66 & 315 & 2 & 2 \\
\hline MT-LB/SA-13 & $\mathrm{N}$ & 11900 & 62 & 60 & 264 & 2 & 2 \\
\hline YW531 (PRC) & $\mathrm{N}$ & 12600 & 65 & 60 & 353 & 2 & 2 \\
\hline BMD-2 & $N$ & 8225 & 60 & 60 & 265 & 2 & 2 \\
\hline $\begin{array}{l}\text { Representative } \\
2 \text { Representative } \\
\text { Representative }\end{array}$ & $\begin{array}{l}\text { for WARSI } \\
\text { for WARSI } \\
\text { for CCTT-S }\end{array}$ & $\begin{array}{l}\text { M \& JWARS by ER } \\
M \text { selected by AMS } \\
\text { AAF. }\end{array}$ & $\begin{array}{l}\text { DC, October } 20 \\
\text { AA, October } 19\end{array}$ & $\begin{array}{l}000 . \\
998 .\end{array}$ & & & \\
\hline
\end{tabular}




\section{Appendix C Wheeled Vehicle Analysis}


Response: Tac Stnd (kph)

\begin{tabular}{|c|c|c|c|c|c|c|}
\hline Summary of Fit & & \multirow{2}{*}{\multicolumn{2}{|c|}{0851013}} & & & \\
\hline RSquare & & & & & & \\
\hline RSquare Adj & & \multicolumn{2}{|c|}{0.776519} & & & \\
\hline Root Mean Square & & \multicolumn{2}{|c|}{3.589956} & & & \\
\hline Mean of Response & & \multicolumn{2}{|c|}{35.12} & & & \\
\hline \multicolumn{2}{|c|}{ Observations (or Sum Wgts) } & \multicolumn{2}{|r|}{10} & & & \\
\hline Parameter Estimates & \multirow{2}{*}{\multicolumn{2}{|c|}{ Estimate }} & \multirow[b]{2}{*}{ Std Error } & \multirow[b]{2}{*}{ t Ratio } & \multirow{2}{*}{ Prob $>|t|$} & \\
\hline Term & & & & & & \\
\hline Intercept & \multicolumn{2}{|c|}{2.5042159} & 11.07715 & 0.23 & 0.8287 & \\
\hline Power-to- & \multicolumn{2}{|c|}{1.1867991} & 0.213789 & 5.55 & 0.0014 & \\
\hline Max Gradient \% & \multicolumn{2}{|c|}{0.4109265} & 0.167342 & 2.46 & 0.0494 & \\
\hline Mfg Type Code & \multicolumn{2}{|c|}{-8.031146} & 2.925632 & -2.75 & 0.0335 & \\
\hline \multicolumn{7}{|l|}{ Effect Test } \\
\hline Source & Nparm & DF & \multicolumn{2}{|c|}{ Sum of Squares } & F Ratio & Prob $>F$ \\
\hline Power-to- & 1 & 1 & \multicolumn{2}{|c|}{397.15584} & 30.8165 & 0.0014 \\
\hline Max Gradient \% & 1 & 1 & \multicolumn{2}{|c|}{77.71398} & 6.0300 & 0.0494 \\
\hline Mfg Type Code & 1 & 1 & \multicolumn{2}{|c|}{97.11673} & 7.5356 & 0.0335 \\
\hline
\end{tabular}

Figure C1. Results of regression analysis for wheeled vehicles (Iteration 1)

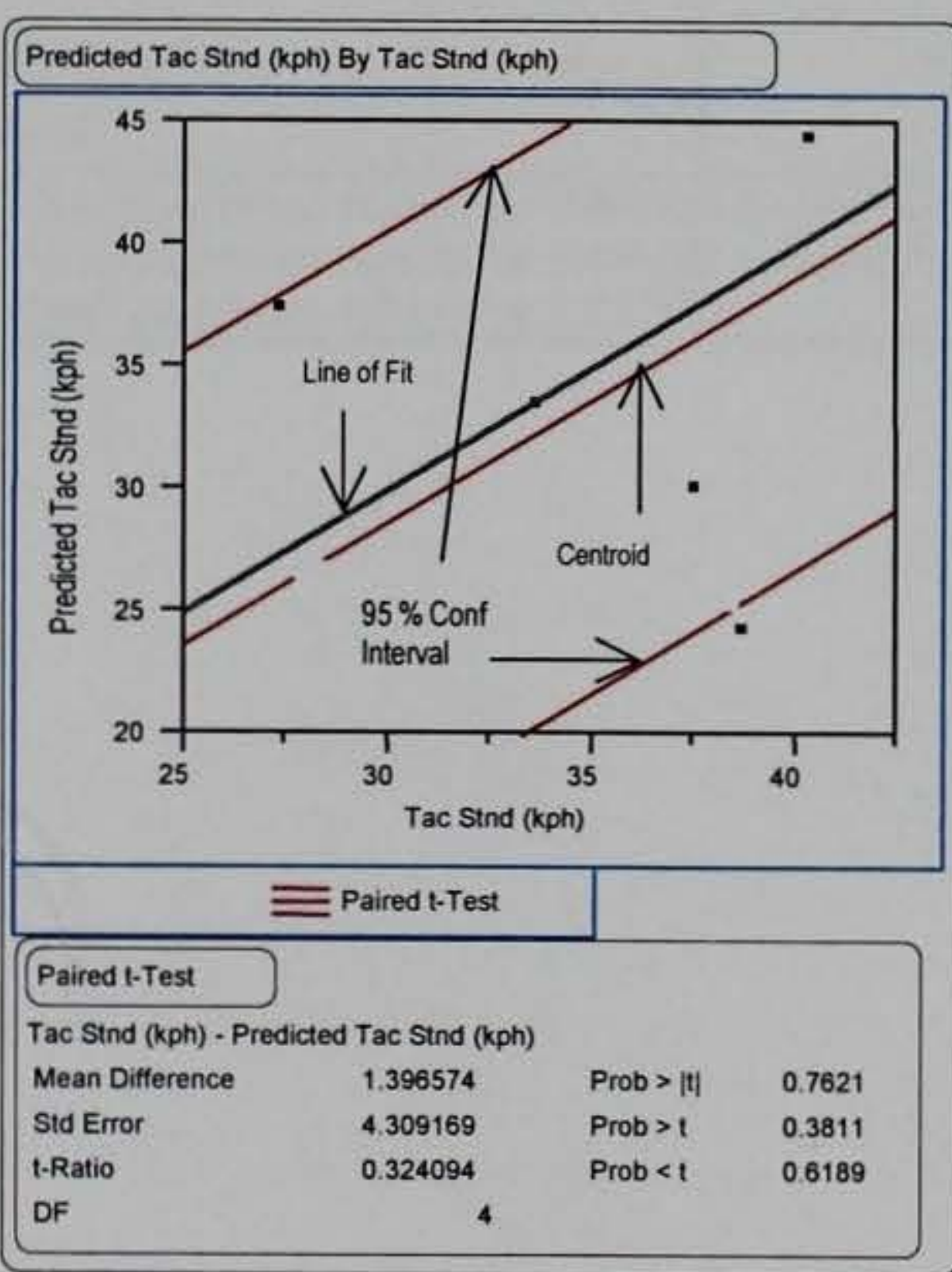

Figure C2. Results of paired t test for wheeled vehicles (Iteration 1) 


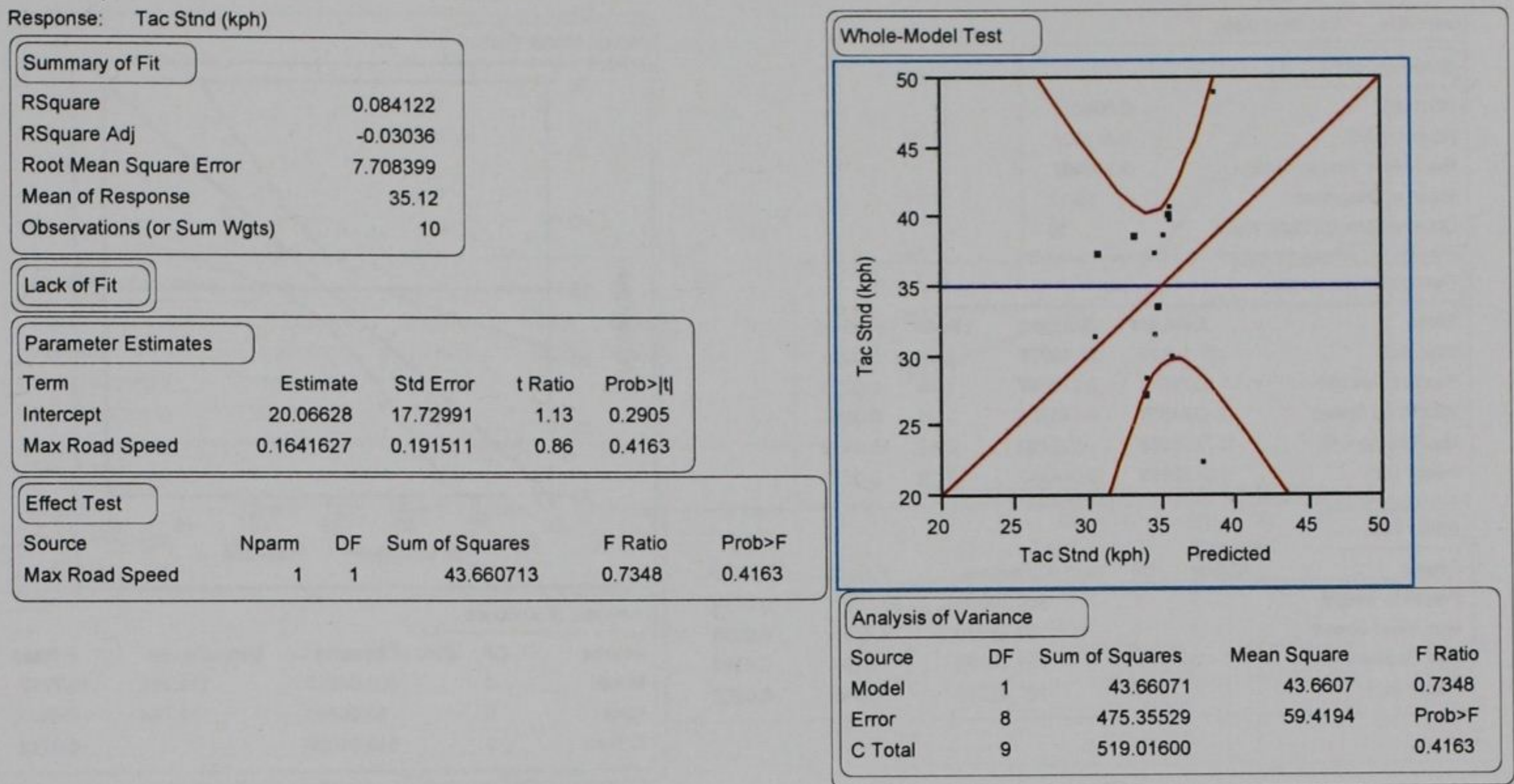

Figure C3. Results of regression analysis for wheeled vehicles (Iteration 2)

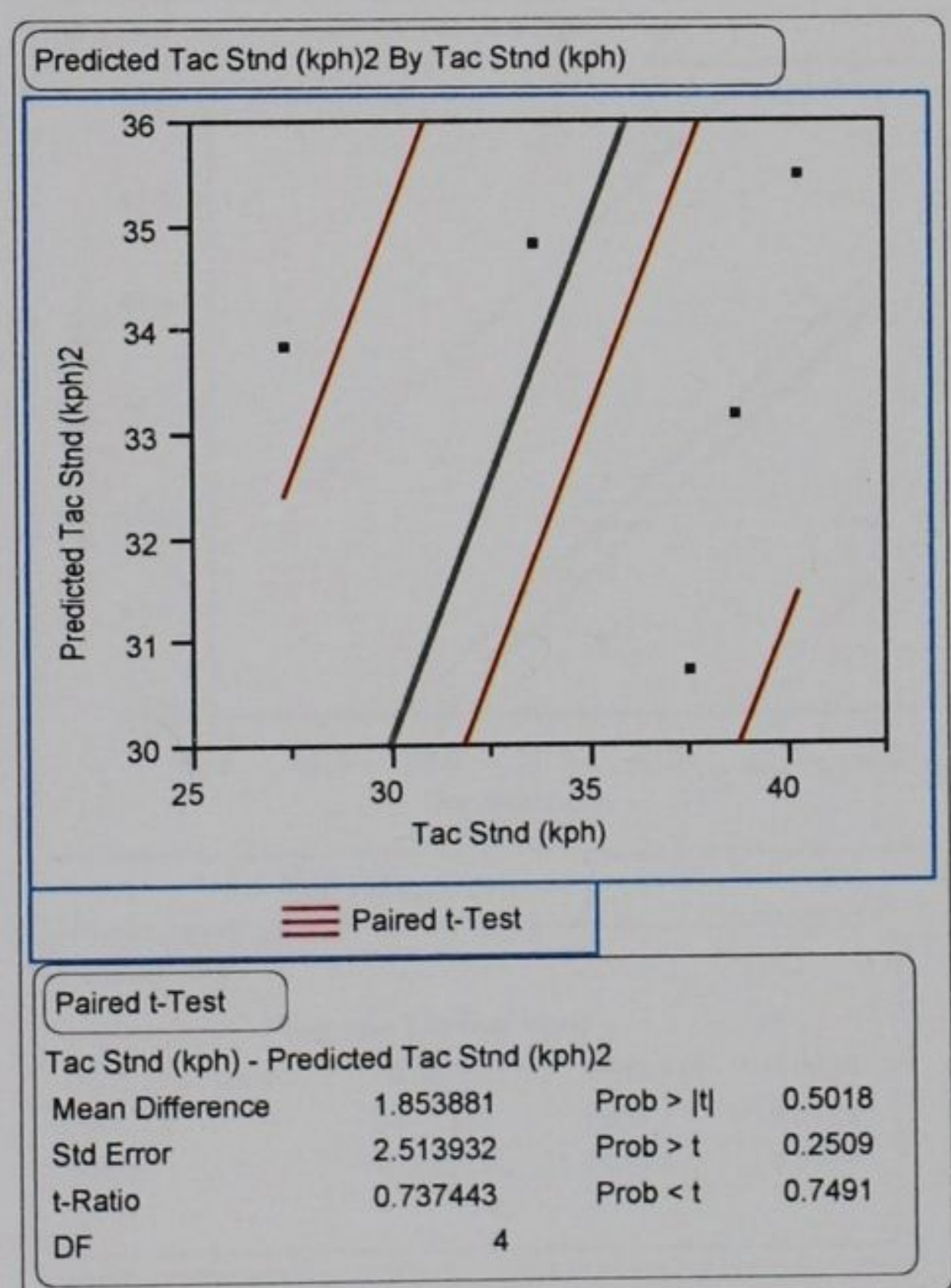

Figure C4. Results of paired t test for wheeled vehicles (Iteration 2) 


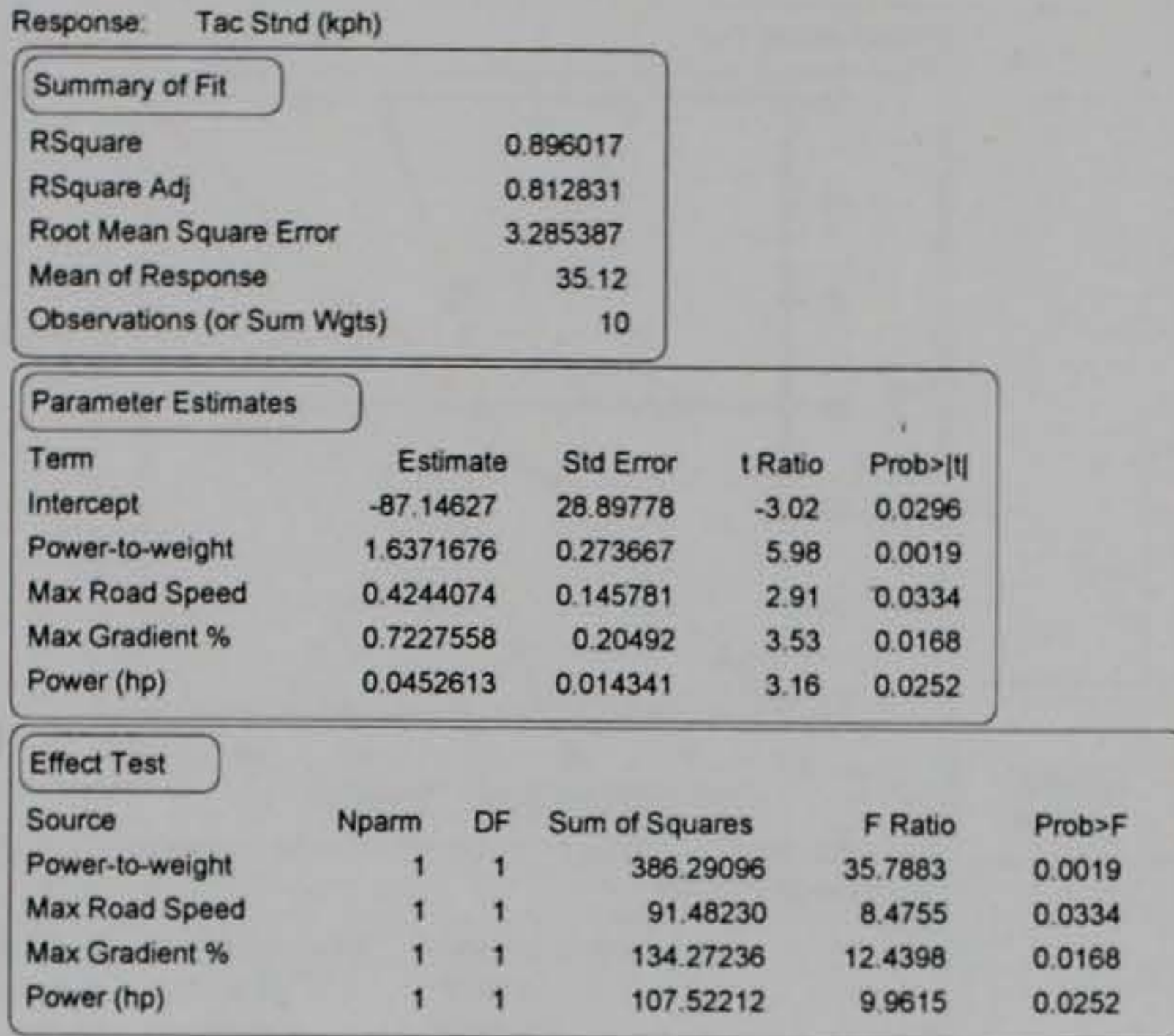

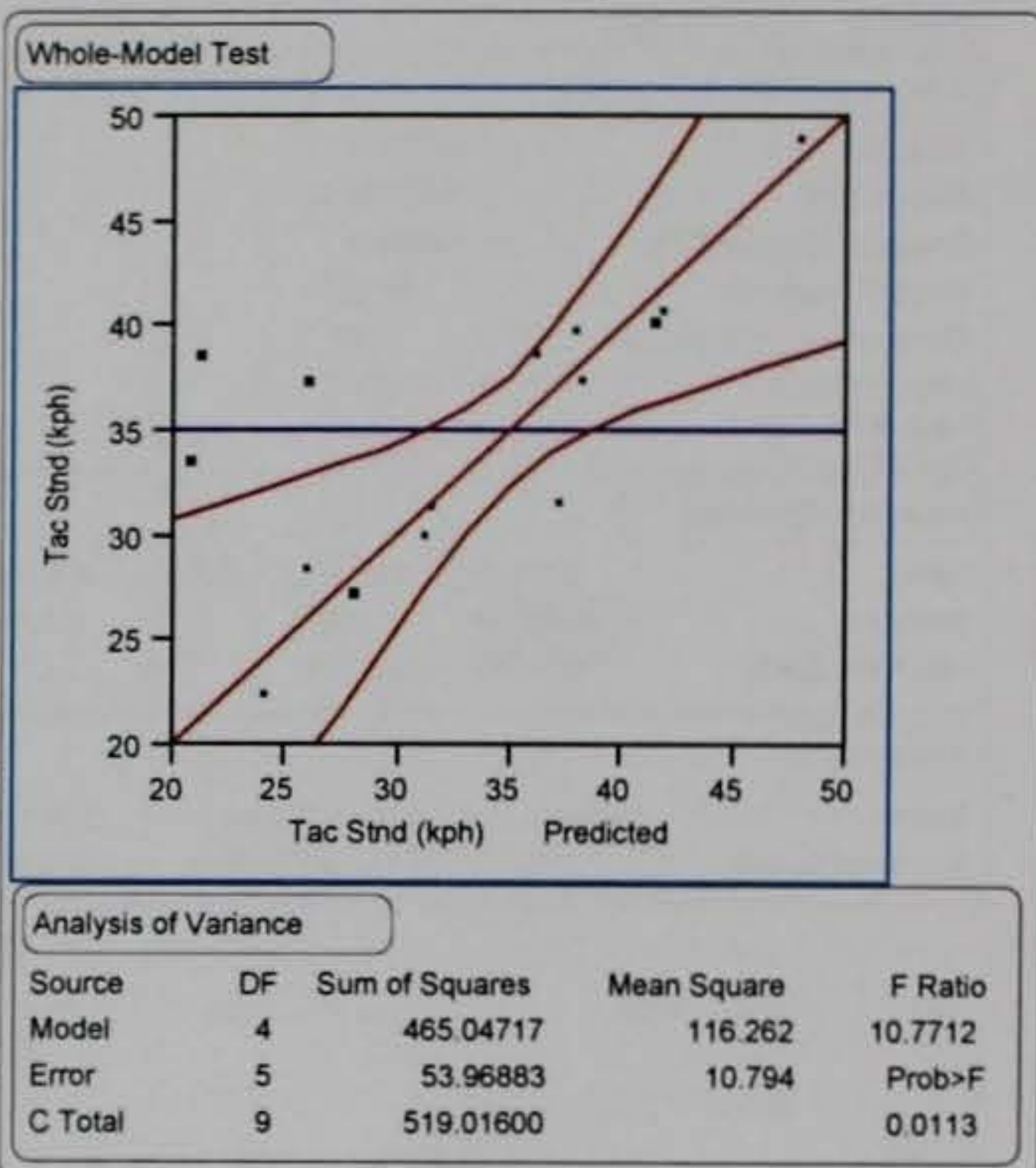

Figure C5. Results of regression analysis for wheeled vehicles (Iteration 3 )

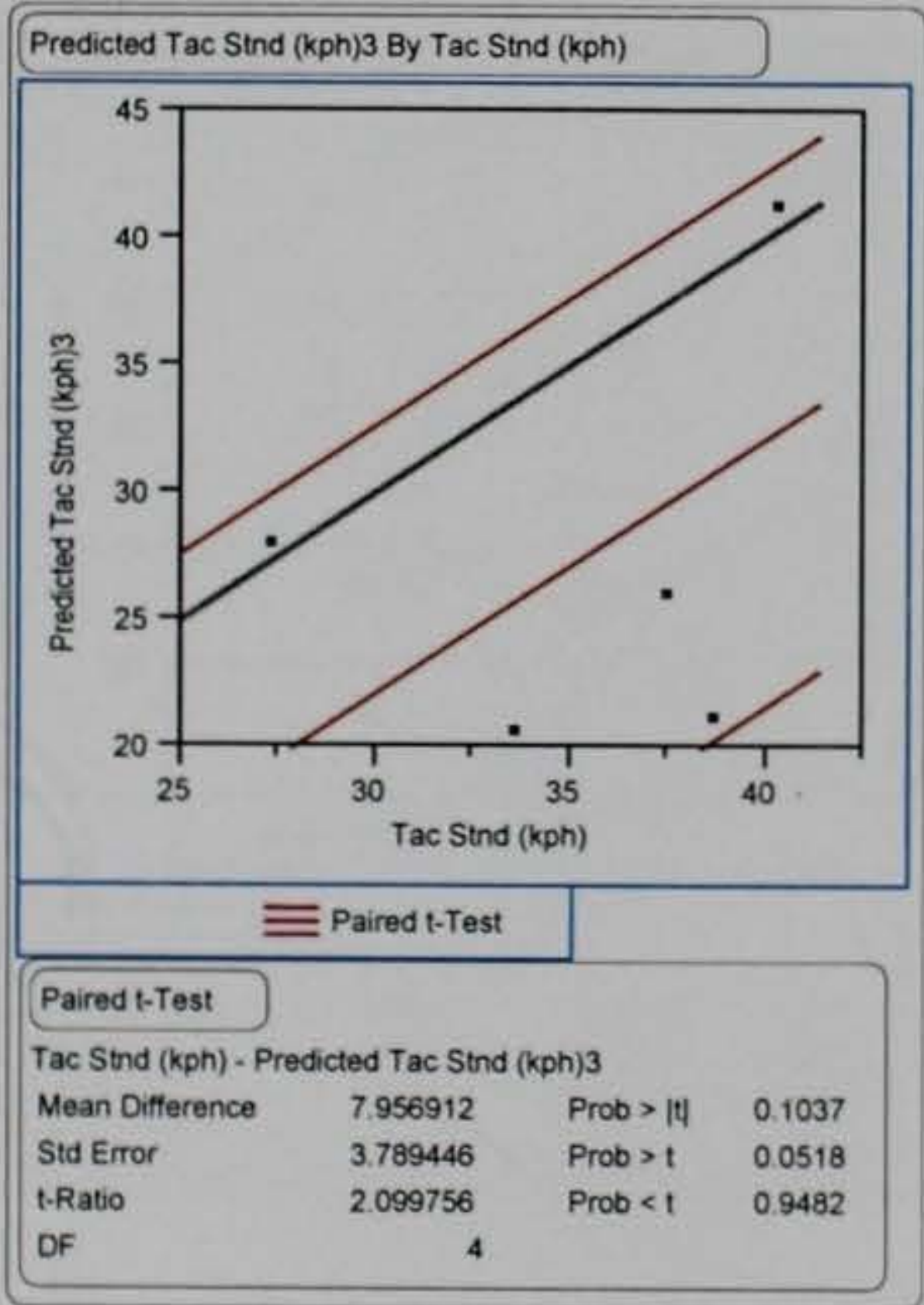

Figure C6. Results of paired $t$ test for wheeled vehicles (Iteration 3 ) 


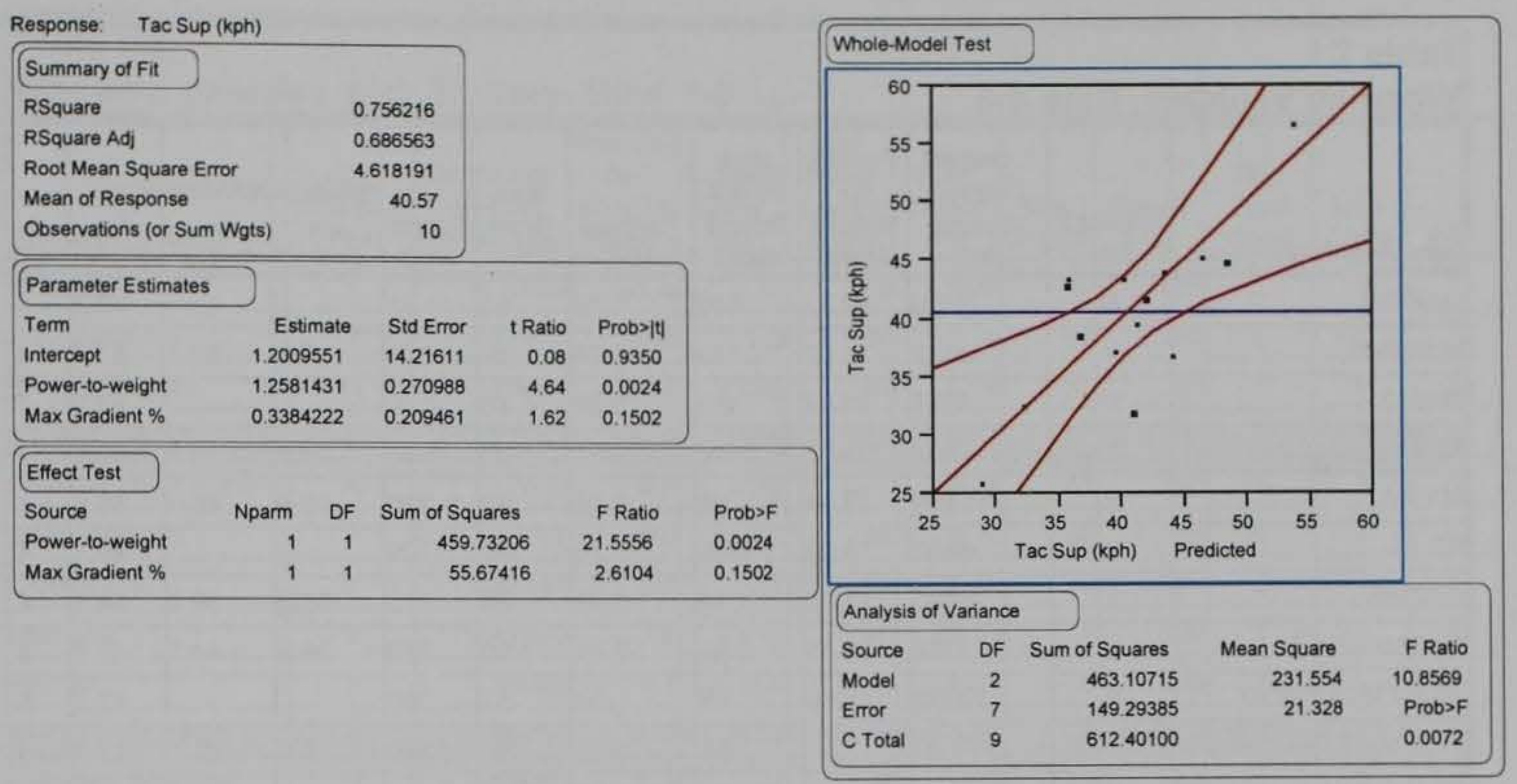

Figure C7. Results of regression analysis for wheeled vehicles (Iteration 4)

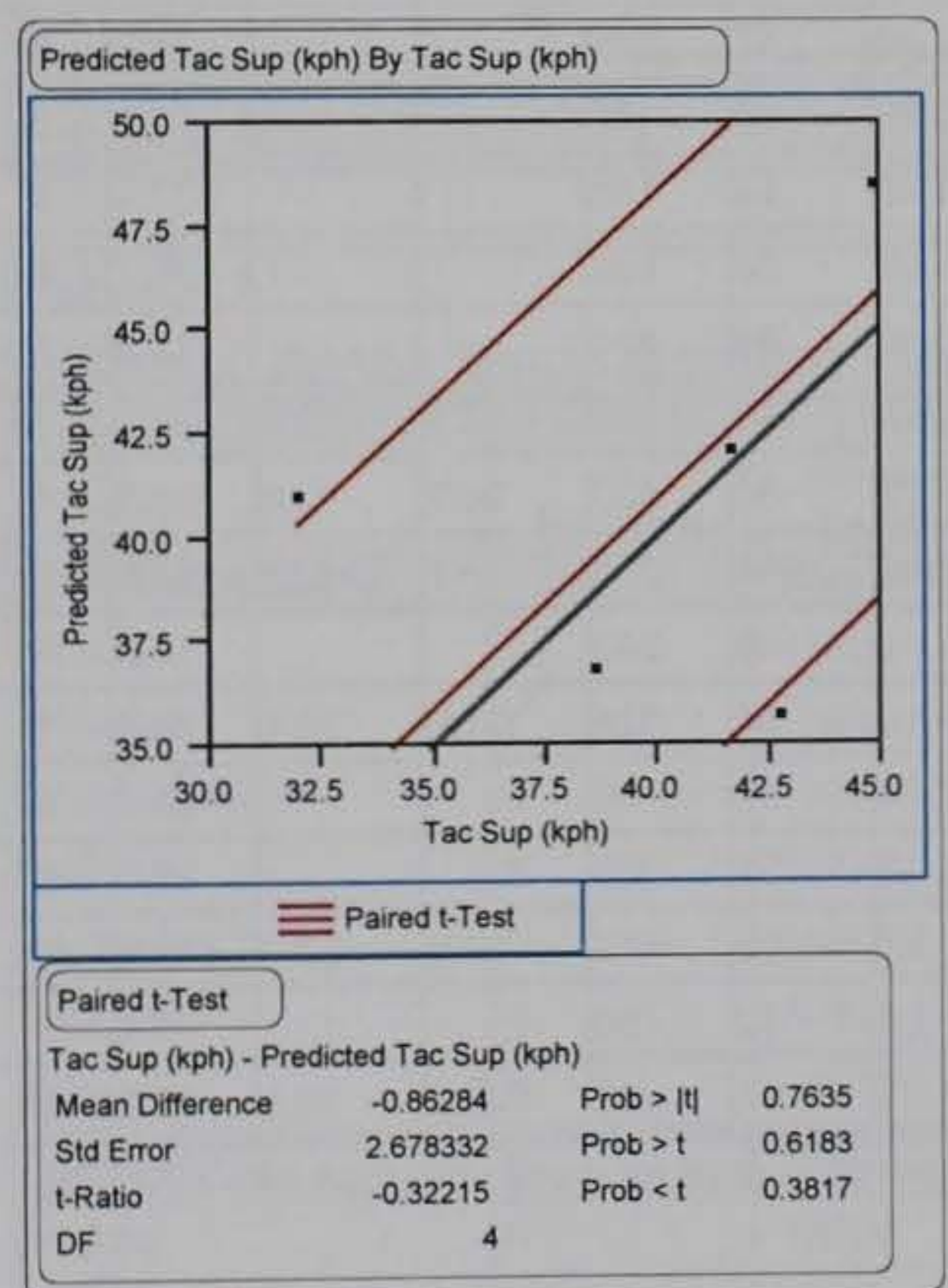

Figure C8. Results of paired t test for wheeled vehicles (Iteration 4) 


\begin{tabular}{|c|c|c|c|c|c|c|c|c|c|c|c|}
\hline \multicolumn{12}{|c|}{$\begin{array}{l}\text { Table C1 } \\
\text { Wheeled Vehicles, Bins 4-6 }\end{array}$} \\
\hline Wheeled Vehicle & \begin{tabular}{c|} 
NRMM II \\
File
\end{tabular} & $\begin{array}{c}\text { Combat/ } \\
\text { Loaded } \\
\text { Weight } \\
(\mathrm{kg})\end{array}$ & $\begin{array}{c}\text { Power- } \\
\text { to- } \\
\text { Weight } \\
\text { Ratio } \\
\end{array}$ & $\begin{array}{c}\text { Max } \\
\text { Road } \\
\text { Speed } \\
\text { (kph) }\end{array}$ & $\begin{array}{c}\text { Fording } \\
(\mathrm{m})\end{array}$ & \begin{tabular}{c|} 
Max \\
Grad \\
$\%$
\end{tabular} & $\begin{array}{c}\text { Power } \\
\text { (hp) }\end{array}$ & \begin{tabular}{|} 
NRMMII \\
$Y_{\text {Ts }}$ \\
$(\mathrm{kph})$
\end{tabular} & $\begin{array}{c}\text { NRMMIII } \\
\text { Yss } \\
\text { (kph) }\end{array}$ & $\begin{array}{c}\text { Pred. } \\
\text { Yss } \\
\text { (kph) }\end{array}$ & BIN \\
\hline UAZ469 & $\mathrm{N}$ & 2290 & 29.71 & 100 & 0.70 & 62 & 75 & & & \begin{tabular}{|l|}
59.7 \\
\end{tabular} & 4 \\
\hline M1025A2 ${ }^{1}$ & $Y$ & 4672 & 31.07 & 113 & 0.76 & 40 & 160 & 49.1 & 56.7 & 53.9 & 4 \\
\hline M1043 & $\mathrm{N}$ & 4672 & 31.07 & 113 & 0.76 & 40 & 160 & & & 53.9 & 4 \\
\hline M1078 & $Y$ & 9507 & 21.47 & 94 & 0.81 & 60 & 225 & 40.3 & 44.9 & 48.7 & 4 \\
\hline M1083 & $Y$ & 13258 & 19.84 & 94 & 0.81 & 60 & 290 & 40.9 & 45.4 & 46.6 & 4 \\
\hline RB 44 & $\mathrm{~N}$ & 5300 & 18.66 & 109 & 0.75 & 60 & 109 & & & 45.1 & 4 \\
\hline GAZ-66 & $Y$ & 5800 & 17.99 & 95 & 0.80 & 60 & 115 & 30.2 & 36.9 & 44.3 & 4 \\
\hline M1084/MTV ${ }^{2,3}$ & $Y$ & 15078 & 17.45 & 94 & 0.81 & 60 & 290 & 39.9 & 44.1 & 43.6 & 4 \\
\hline BAZ-135L4/FROG & $\mathrm{N}$ & 19000 & 17.19 & 65 & 0.58 & 57 & 360 & & & 42.2 & 5 \\
\hline ZIL 135/FROG7 & $Y$ & 19000 & 17.19 & 65 & 0.58 & 57 & 360 & 37.5 & 41.7 & 42.2 & 5 \\
\hline MAN Cat I A1 & $\mathrm{N}$ & 32000 & 15.59 & 90 & 1.20 & 60 & 550 & & & 41.2 & 5 \\
\hline M923 $3^{3}$ & $\bar{Y}$ & 14030 & 15.52 & 84 & 0.76 & 60 & 240 & 27.3 & 32.0 & 41.2 & 5 \\
\hline M977 HEMTT $^{1}$ & $Y$ & 27080 & 14.91 & 88 & 0.76 & 60 & 445 & 37.6 & 43.5 & 40.4 & 5 \\
\hline M985 HEMTT $^{2}$ & $Y$ & 28168 & 14.33 & 88 & 0.76 & 60 & 445 & 31.8 & 37.2 & 39.7 & 5 \\
\hline MAZ543M/Scud/SA10 & $Y$ & 32470 & 14.60 & 63 & 1.10 & 57 & 525 & & & 38.9 & 5 \\
\hline SEE & $\mathrm{N}$ & 7250 & 13.76 & 80 & 0.76 & 60 & 110 & & & 38.9 & 5 \\
\hline ZIL 131 & $\mathrm{~N}$ & 10425 & 13.05 & 80 & 1.40 & 58 & 150 & & & 37.4 & 6 \\
\hline URAL375/SA-4Reload & $\mathrm{N}$ & 13300 & 12.28 & 75 & 1.00 & 60 & 180 & & & 37.1 & 6 \\
\hline RM70 & $\mathrm{N}$ & 25300 & 9.68 & 75 & 1.40 & 60 & 270 & & & 37.0 & 6 \\
\hline M35A2 & $Y$ & 10400 & 12.21 & 90 & 0.76 & 60 & 140 & 33.6 & 38.6 & 37.0 & 6 \\
\hline M1074/PLS & $Y$ & 39916 & 11.36 & 91 & 1.22 & 60 & 500 & 38.9 & 43.6 & 35.9 & 6 \\
\hline KRAZ 260V & $Y$ & 22000 & 11.88 & 80 & 1.20 & 58 & 288 & 38.7 & 42.8 & 35.9 & 6 \\
\hline ZTS 152 & $\mathrm{~N}$ & 29250 & 13.74 & 80 & 1.40 & 60 & 345 & & & 35.0 & 6 \\
\hline MAZ543A & $\mathrm{N}$ & 43300 & 11.00 & 63 & 1.10 & 57 & 525 & 31.6 & 39.6 & 34.4 & 6 \\
\hline ZIL 157 & $\mathrm{~N}$ & 8450 & 11.70 & 65 & 0.85 & 53 & 109 & & & 34.0 & 6 \\
\hline KRAZ 214 & $\mathrm{~N}$ & 19300 & 9.64 & 55 & 1.00 & 57 & 205 & & & 32.7 & 6 \\
\hline MK48/14 & $Y$ & 47628 & 8.48 & 84 & 1.52 & 60 & 445 & 28.6 & 32.6 & 32.3 & 6 \\
\hline TAM 150 T11 & $\mathrm{N}$ & 11400 & 11.94 & 85 & 1.00 & 43 & 150 & & & 30.8 & 6 \\
\hline${\mathrm{M} 917^{2}}^{2}$ & $Y$ & 33070 & 10.97 & 107 & 0.61 & 41 & 400 & 22.6 & 26.1 & 29.0 & 6 \\
\hline $\begin{array}{l}\text { Note: Grayed rows indi } \\
\text { prediction and the regre } \\
\text { 1 Representative vehicl } \\
\text { 2 Representative vehic } \\
\text { 3 Representative vehic } \\
\text { Repen }\end{array}$ & for WAI & $\begin{array}{l}\text { not part } \\
\text { ion. }\end{array}$ & RS by ER & RDC, Octc & er 200 & & d for $c$ & cross v & tion wit & a NRM & \\
\hline
\end{tabular}


Table C2

Wheeled Vehicles with Trailers, Bins 7-9

\begin{tabular}{|c|c|c|c|c|c|c|c|c|c|c|c|}
\hline Wheeled Vehicle & $\begin{array}{c}\text { NRMM II } \\
\text { File } \\
\end{array}$ & $\begin{array}{c}\text { Combined } \\
\text { Vehicle } \\
\text { Weight }(\mathrm{kg})\end{array}$ & \begin{tabular}{|c|} 
Power- \\
to- \\
Weight \\
Ratio \\
\end{tabular} & $\begin{array}{c}\text { Ground } \\
\text { Clear } \\
\text { (m) }\end{array}$ & $\begin{array}{c}\text { Max } \\
\text { Road } \\
\text { Speed } \\
(\mathrm{kph}) \\
\end{array}$ & $\begin{array}{c}\text { Ford } \\
\text { Depth } \\
(\mathrm{m}) \\
\end{array}$ & $\begin{array}{c}\text { Max } \\
\text { Grad\% }\end{array}$ & $\begin{array}{c}\text { Power } \\
\text { (hp) }\end{array}$ & $\begin{array}{l}\text { Mfg } \\
\text { Type } \\
\text { Code } \\
\end{array}$ & $\begin{array}{c}\text { Use } \\
\text { Code }\end{array}$ & BIN \\
\hline $\mathrm{M} 1025 \mathrm{~A} 2 \mathrm{t}$ & $Y$ & 6668 & 21.6 & 0.38 & 113 & 0.76 & 40 & 160 & 1 & 1 & 7 \\
\hline M923w/trailer ${ }^{1}$ & $Y$ & 12977 & 16.6 & 0.3 & 84 & 0.76 & 60 & 240 & 1 & 1 & 7 \\
\hline M813 w/ trailer & $\mathrm{N}$ & 18985 & 11.4 & 0.295 & 84 & 0.76 & 67 & 240 & 1 & 1 & 7 \\
\hline MTVM1094² & $Y$ & 25049 & 10.4 & 0.559 & 94 & 0.81 & 60 & 290 & 1 & 1 & 7 \\
\hline Leyland Truck $(8 \times 6)$ & $\mathrm{N}$ & 32000 & 9.8 & 0.29 & 75 & 0.75 & 61 & 350 & 1 & 1 & 8 \\
\hline M985 w/trailer ${ }^{2}$ & $Y$ & 42121 & 9.5 & 0.3 & 88 & 0.76 & 60 & 445 & 1 & 1 & 8 \\
\hline TATRA815 & Y & 35400 & 8.4 & 0.41 & 80 & 1.40 & 30 & 333 & 2 & 1 & 8 \\
\hline M915A2 & $Y$ & 47670 & 7.6 & 0.254 & 90 & 0.51 & 18.4 & 400 & 1 & 1 & 8 \\
\hline M916A1 & $Y$ & 59020 & 6.1 & 0.305 & 85.3 & 0.51 & 18 & 400 & 1 & 1 & 8 \\
\hline MAZ-537 w/trailer & $\mathrm{N}$ & 86600 & 5.5 & 0.35 & 50 & 1.30 & 8 & 525 & 2 & 3 & 9 \\
\hline Hanyang HY473A & $\mathrm{N}$ & 62000 & 5.2 & 0.34 & 64 & 0.70 & 24 & 355 & 2 & 3 & 9 \\
\hline Oshkosh M1070 & $\mathrm{N}$ & 104961 & 4.3 & 0.33 & 72 & 0.71 & 15 & 500 & 1 & 3 & 9 \\
\hline Mercedes Benz 3850 & $\mathrm{~N}$ & 110000 & 4.1 & 0.39 & 85 & 0.70 & 32 & 500 & 1 & 3 & 9 \\
\hline M911 HET $^{1,2}$ & Y & 102514 & 4.0 & 0.25 & 72 & 1.07 & 20 & 450 & 1 & 3 & 9 \\
\hline FAP 3232 & $\mathrm{~N}$ & 81000 & 3.6 & 0.38 & 60 & 1.20 & 32 & 320 & 2 & 3 & 9 \\
\hline
\end{tabular}

Table C3

Wheeled Amphibious Combat Vehicles, Bin 11

\begin{tabular}{|c|c|c|c|c|c|c|c|c|}
\hline Wheeled Vehicle & $\begin{array}{c}\text { NRMM II } \\
\text { File }\end{array}$ & $\begin{array}{l}\text { Combat } \\
\text { Loaded } \\
\text { Weight }(\mathbf{k g})\end{array}$ & $\begin{array}{c}\text { Ground } \\
\text { Clear }(m)\end{array}$ & $\begin{array}{c}\text { Max } \\
\text { Road } \\
\text { Speed } \\
\text { (kph) }\end{array}$ & $\left|\begin{array}{c}\text { Max } \\
\text { Grad \% }\end{array}\right|$ & $\begin{array}{c}\text { Power } \\
\text { (hp) }\end{array}$ & $\begin{array}{c}\text { Mfg Type } \\
\text { Code }\end{array}$ & $\begin{array}{c}\text { Primary Use } \\
\text { Code }\end{array}$ \\
\hline BTR70 & $\mathrm{Y}$ & 11500 & 0.48 & 80 & 60 & 264 & 2 & 2 \\
\hline BTR60P & $Y$ & 9980 & 0.48 & 80 & 60 & 180 & 2 & 2 \\
\hline BRDM-2/ SA-9 & $\mathrm{Y}$ & 7000 & 0.43 & 100 & 60 & 154 & 2 & 2 \\
\hline LAV25 & $\mathrm{Y}$ & 13400 & 0.39 & 100 & 60 & 303 & 1 & 2 \\
\hline $\mathrm{AMX} 10 \mathrm{RC}$ & $\mathrm{N}$ & 15880 & 0.35 & 85 & 50 & 300 & 1 & 2 \\
\hline BAZ-5937 & $\mathrm{N}$ & 10650 & 0.43 & 70 & 60 & 295 & 2 & 2 \\
\hline BOV & $\mathrm{N}$ & 5700 & 0.33 & 95 & 55 & 163 & 2 & 2 \\
\hline BTR 152 & $\mathrm{~N}$ & 8950 & 0.30 & 75 & 55 & 121 & 2 & 2 \\
\hline BTR80 & $\mathrm{N}$ & 13600 & 0.48 & 90 & 60 & 286 & 2 & 2 \\
\hline BTR90 & $\mathrm{N}$ & 17000 & 0.53 & 90 & 60 & 500 & 2 & 2 \\
\hline Fuchs/M93 NBC & $\mathrm{N}$ & 17000 & 0.41 & 105 & 70 & 320 & 1 & 2 \\
\hline LAV600 & $\mathrm{N}$ & 18500 & 0.53 & 100 & 60 & 298 & 1 & 2 \\
\hline Panhard VCR & $\mathrm{N}$ & 7900 & 0.38 & 90 & 60 & 160 & 1 & 2 \\
\hline SA-8 & $\mathrm{N}$ & 9000 & 0.40 & 60 & 60 & 175 & 2 & 2 \\
\hline TAB-71 & $\mathrm{N}$ & 11000 & 0.47 & 95 & 60 & 308 & 2 & 2 \\
\hline TAB-77 & $\mathrm{N}$ & 13350 & 0.53 & 83 & 60 & 291 & 2 & 2 \\
\hline WZ 551 & $\mathrm{~N}$ & 15000 & 0.41 & 85 & 60 & 282 & 2 & 2 \\
\hline
\end{tabular}




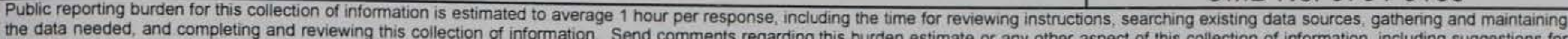

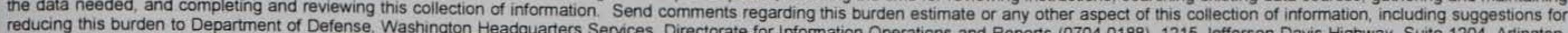

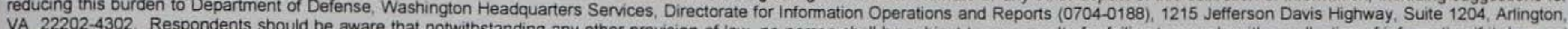

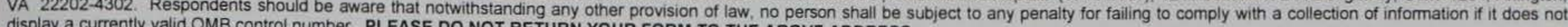
display a currently valid OMB control number. PLEASE DO NOT RETURN YOUR FORM TO THE ABOVE ADDRESS.

\begin{tabular}{l|l|l}
\hline $\begin{array}{l}\text { 1. REPORT DATE (DD-MM-YYYY) } \\
\text { November } 2002\end{array}$ & $\begin{array}{l}\text { 2. REPORT TYPE } \\
\text { Final report }\end{array}$ & 3. DATES COVERED (FrOm - To)
\end{tabular}

4. TITLE AND SUBTITLE

Procedure for Categorizing Ground Vehicles

5a. CONTRACT NUMBER

5b. GRANT NUMBER

5c. PROGRAM ELEMENT NUMBER

6. AUTHOR(S)

E. Alex Baylot, Burhman Q. Gates

5d. PROJECT NUMBER

5e. TASK NUMBER

5f. WORK UNIT NUMBER

8. PERFORMING ORGANIZATION REPORT NUMBER

U.S. Army Engineer Research and Development Center

Geotechnical and Structures Laboratory

ERDC/GSL TR-02-21

3909 Halls Ferry Road

Vicksburg, MS 39180-6199

\section{SPONSORING / MONITORING AGENCY NAME(S) AND ADDRESS(ES)}

10. SPONSOR/MONITOR'S ACRONYM(S)

Office of Assistant Secretary of the Army for Acquisition, Logistics, and Technology HQ Dept. of Army, ATTN: SAAL-ZT Rm 3E620, 103 Pentagon, Washington, DC 20310; Army Models and Simulation Office

HQDA, DCS G3, (DAMO-ZS), 400 Army Pentagon, Washington, DC 20310-0400

\section{DISTRIBUTION / AVAILABILITY STATEMENT}

Approved for public release; distribution is unlimited.

\section{SUPPLEMENTARY NOTES}

\section{ABSTRACT}

This report presents the procedures and findings of categorizing ground vehicles while providing the Modeling and Simulation (M\&S) community with a set of common categories and common representative vehicles. The procedure successfully used currently available parameterizations for ground vehicles and then used a subset of those vehicles to represent other vehicles with similar mobility performances. This was accomplished by defining the categorization, devising a practical binning system, and sorting the vehicles into those bins. A sensitivity analysis was performed to evaluate the robustness of the categorization mechanism against changes in the values of vehicle input parameters. Existing bins and representative vehicles were taken from WARSIM 2000 and JWARS. New bins were named for vehicles that defied classification into those bins. The resulting set of bins is provided in this report and includes the new groups of amphibious tracked, amphibious wheeled vehicles, and small vehicles $(<500 \mathrm{~kg})$.

Based on the findings of this research, it is recommended that the 12 bins for categorizing ground vehicles be accepted as a standard for the M\&S community and be accepted for the U.S. Army Standards Repository System and the M\&S Resource Repository. It is further recommended that current and future wargame simulations and decision support systems adopt the categorization procedure and bins documented within this report.

(Continued)

\section{SUBJECT TERMS}

See reverse.

16. SECURITY CLASSIFICATION OF:
\begin{tabular}{l|l|l|}
\hline $\begin{array}{l}\text { a. REPORT } \\
\text { UNCLASSIFIED }\end{array}$ & b. ABSTRACT & c. THIS PAGE \\
\end{tabular}

17. LIMITATION OF ABSTRACT 18. NUMBER
OF PAGES

62 19a. NAME OF RESPONSIBLE PERSON

19b. TELEPHONE NUMBER (include area code) 


\section{4. (Concluded).}

Further refinement of this procedure may be accomplished by obtaining a larger set of vehicle data and predicting mobility on a larger set of terrain databases. Additionally, prior to analysis or during the course of simulation, allowing the flexibility of changing the representative vehicles of bins as a function of terrain conditions and features (i.e., plains, hills, obstacles) would better model the performance of a particular vehicle without being vehicle specific. This is recommended as the next level of resolution increase.

\section{5. (Concluded).}

Bins

Categories

CBTXXI

CombatXXI

Computer generated forces

JointSAF

JSAF

Mobility

Models

OneSAF

OTB

SAF

SAFOR

Semi-automated forces

Simulations

STNDMob

Vehicle

War games

WARSIM 